\title{
Multi-step extragradient method with regularization for triple hierarchical variational inequalities with variational inclusion and split feasibility constraints
}

\author{
Lu-Chuan Ceng ${ }^{1}$, Chin-Tzong Pang ${ }^{2 *}$ and Ching-Feng Wen ${ }^{3}$
}

\author{
${ }^{*}$ Correspondence: \\ imctpang@saturn.yzu.edu.tw \\ ${ }^{2}$ Department of Information \\ Management, Innovation Center for \\ Big Data and Digital Convergence, \\ Yuan Ze University, Chung-Li, 32003, \\ Taiwan \\ Full list of author information is \\ available at the end of the article
}

\begin{abstract}
By combining Korpelevich's extragradient method, the viscosity approximation method, the hybrid steepest-descent method, Mann's iteration method, and the gradient-projection method with regularization, a hybrid multi-step extragradient algorithm with regularization for finding a solution of triple hierarchical variational inequality problem is introduced and analyzed. It is proven that under appropriate assumptions, the proposed algorithm converges strongly to a unique solution of a triple hierarchical variational inequality problem which is defined over the set of solutions of a hierarchical variational inequality problem defined over the set of common solutions of finitely many generalized mixed equilibrium problems (GMEP), finitely many variational inclusions, fixed point problems, and the split feasibility problem (SFP). We also prove the strong convergence of the proposed algorithm to a common solution of the SFP, finitely many GMEPs, finitely many variational inclusions, and the fixed point problem of a strict pseudocontraction. The results presented in this paper improve and extend the corresponding results announced by several others.

MSC: Primary 49J30; 47H09; secondary 47J20; 49M05
\end{abstract}

Keywords: triple hierarchical variational inequalities; hierarchical variational inequalities; variational inclusions; split feasibility problems; fixed point problems; iterative algorithm; strong convergence result

\section{Introduction}

The following problems have their own importance because of their applications in diverse areas of science, engineering, social sciences, and management:

- Equilibrium problems including variational inequalities.

- Variational inclusion problems.

- Split feasibility problems.

- Fixed point problems.

One way or the other, these problems are related to each other. They are described as follows. 


\section{Equilibrium problem}

Let $C$ be a nonempty closed convex subset of a real Hilbert space $H$ and $\Theta: C \times C \rightarrow \mathbb{R}$ be a real-valued bifunction. The equilibrium problem (EP) is to find an element $x \in C$ such that

$$
\Theta(x, y) \geq 0, \quad \forall y \in C
$$

The set of solutions of EP is denoted by $\operatorname{EP}(\Theta)$. It includes several problems, namely, variational inequality problems, optimization problems, saddle point problems, fixed point problems, etc., as special cases. For further details on EP, we refer to [1-6] and the references therein.

Let $A: C \rightarrow H$ be a nonlinear operator. If $\Theta(x, y)=\langle A(x), y-x\rangle$, then EP reduces to the variational inequality problem of finding $x \in C$ such that

$$
\langle A(x), y-x\rangle \geq 0, \quad \forall y \in C
$$

For further details on variational inequalities and their generalizations, we refer to [7-13] and the references therein.

During the last two decades, EP has been extended and generalized in several directions. The generalized mixed equilibrium problem (GMEP), one of the generalizations of EP, is to find $x \in C$ such that

$$
\Theta(x, y)+\varphi(y)-\varphi(x)+\langle A x, y-x\rangle \geq 0, \quad \forall y \in C
$$

where $\varphi: C \rightarrow \mathbb{R}$ is a real-valued function. The set of solutions of GMEP is denoted by $\operatorname{GMEP}(\Theta, \varphi, A)$. For different choices of operators/functions $\Theta, \varphi$, and $A$, we get different forms of equilibrium problems. For applications of GMEP, we refer to $[14,15]$ and the references therein.

\section{Variational inclusion problem}

Let $B: C \rightarrow H$ be a single-valued mapping and $R: C \rightarrow 2^{H}$ be a set-valued mapping with $D(R)=C$, where $D(R)$ denotes the domain of $R$. The variational inclusion problem is to find $x \in C$ such that

$$
0 \in B x+R x
$$

We denote by $\mathrm{I}(B, R)$ the solution set of the variational inclusion problem (1.2). In particular, if $B=R=0$, then $\mathrm{I}(B, R)=C$. If $B=0$, then problem (1.2) becomes the inclusion problem introduced by Rockafellar [16]. It is well known that problem (1.2) provides a convenient framework for the unified study of optimal solutions in many optimization related areas including mathematical programming, complementarity problems, variational inequalities, optimal control, mathematical economics, equilibria and game theory, etc. Let a set-valued mapping $R: D(R) \subset H \rightarrow 2^{H}$ be maximal monotone. We define the resolvent operator $J_{R, \lambda}: H \rightarrow \overline{D(R)}$ associated with $R$ and $\lambda>0$ as follows:

$$
J_{R, \lambda}=(I+\lambda R)^{-1}, \quad \forall x \in H .
$$


Huang [17] studied problem (1.2) in the case where $R$ is maximal monotone and $B$ is strongly monotone and Lipschitz continuous with $D(R)=C=H$. Zeng et al. [18] further studied problem (1.2) in a more general setting than in [17]. They gave the geometric convergence rate estimate for approximate solutions. Various types of iterative algorithms for solving variational inclusions have been further studied and developed in the literature; see, for example, [19-22] and the references therein.

\section{Split feasibility problem}

Let $C$ and $Q$ be nonempty closed convex subsets of real Hilbert spaces $\mathcal{H}_{1}$ and $\mathcal{H}_{2}$, respectively. The split feasibility problem (SFP) is to find a point $x$ such that

$$
x \in C \text { and } A x \in Q \text {, }
$$

where $A: \mathcal{H}_{1} \rightarrow \mathcal{H}_{2}$ is a bounded linear operator from $\mathcal{H}_{1}$ to $\mathcal{H}_{2}$. We denote by $\Gamma$ the solution set of the SFP. It is a model of an inverse problem which arises in phase retrievals and in medical image reconstruction. A number of image reconstruction problems can be formulated as SFP; see, for example [23] and the references therein. Recently, it is found that the SFP can also be applied to study intensity-modulated radiation therapy (IMRT); see, for example, $[24,25]$ and the references therein. In the recent past, a wide variety of iterative methods have been proposed to solve SFP; see, for example, [24-28] the references therein.

\section{Fixed point problem}

Let $C$ be a nonempty subset of a $H$ and $T: C \rightarrow C$ be a mapping. The fixed point problem is to find an element $x \in C$ such that $T(x)=x$.

It is a well-known problem and has tremendous applications in different branches of science, engineering, social sciences, and management.

The following proposition provides some relations among the above mentioned problems.

Proposition 1.1 Given $x^{*} \in H$, the following statements are equivalent:

(a) $x^{*}$ solves the SFP;

(b) $x^{*}$ solves the fixed point equation

$$
P_{C}(I-\lambda \nabla f) x^{*}=x^{*},
$$

where $\lambda>0, \nabla f=A^{*}\left(I-P_{Q}\right) A, P_{Q}$ is the projection operator and $A^{*}$ is the adjoint of $A$;

(c) $x^{*}$ solves the variational inequality problem (VIP) of finding $x^{*} \in C$ such that

$$
\left\langle\nabla f\left(x^{*}\right), x-x^{*}\right\rangle \geq 0, \quad \forall x \in C
$$

A variational inequality problem which is defined over the set of fixed points of a mapping is called hierarchical variational inequality problem; that is, when the set $C$ in variational inequality formulation is equal to the set of fixed points of a mapping. A variational inequality problem which is defined over the set of solutions of a hierarchical variational 
inequality problem is called a triple hierarchical variational inequality problem. For further details on hierarchical variational inequality problems and triple hierarchical variational inequality problems, we refer to [29], a recent survey on these problems.

Very recently, Kong et al. [30] considered the following triple hierarchical variational inequality problem (THVIP).

Problem 1.1 Let $C$ be a nonempty closed convex subset of a real Hilbert space $H$ and $F: C \rightarrow H$ be a $\kappa$-Lipschitzian and $\eta$-strongly monotone operator, where $\kappa$ and $\eta$ are positive constants. Let $A: C \rightarrow H$ be a monotone and $L$-Lipschitzian mapping, $V: C \rightarrow$ $H$ be a $\rho$-contraction with coefficient $\rho \in[0,1), S: C \rightarrow C$ be a nonexpansive mapping, and $T: C \rightarrow C$ be a $\xi$-strictly pseudocontractive mapping with $\operatorname{Fix}(T) \cap \operatorname{VI}(C, A) \neq \emptyset$, where $\operatorname{Fix}(T)$ denotes the set of all fixed points of $T$. Let $0<\mu<\frac{2 \eta}{\kappa^{2}}$ and $0<\gamma \leq \tau$, where $\tau=1-\sqrt{1-\mu\left(2 \eta-\mu \kappa^{2}\right)}$. Then the objective is to find $x^{*} \in \Xi$ such that

$$
\left\langle(\mu F-\gamma V) x^{*}, x-x^{*}\right\rangle \geq 0, \quad \forall x \in \Xi
$$

where $\Xi$ denotes the solution set of the hierarchical variational inequality problem (HVIP) of finding $z^{*} \in \operatorname{Fix}(T) \cap \operatorname{VI}(C, A)$ such that

$$
\left\langle(\mu F-\gamma S) z^{*}, z-z^{*}\right\rangle \geq 0, \quad \forall z \in \operatorname{Fix}(T) \cap \operatorname{VI}(C, A) .
$$

Kong et al. [30] presented an algorithm for finding a solution of Problem 1.1. Under some conditions, they proved that the sequence $\left\{x_{n}\right\}$ generated by the proposed algorithm converges strongly to a point $x^{*} \in \operatorname{Fix}(T) \cap \operatorname{VI}(C, A)$ which is a unique solution of Problem 1.1 provided that $\left\{S x_{n}\right\}$ is bounded and $\left\|x_{n+1}-x_{n}\right\|+\left\|x_{n}-z_{n}\right\|=o\left(\epsilon_{n}^{2}\right)$. They also showed under certain conditions that the sequence $\left\{x_{n}\right\}$ generated by proposed algorithm converges strongly to a unique solution $x^{*}$ of the following VIP provided that $\left\|x_{n+1}-x_{n}\right\|+\left\|x_{n}-z_{n}\right\|=o\left(\epsilon_{n}^{2}\right)$ and the sequence $\left\{S x_{n}\right\}$ is bounded:

find $x^{*} \in \Xi$ such that $\left\langle F x^{*}, x-x^{*}\right\rangle \geq 0, \quad \forall x \in \Xi$.

In this paper, we consider the following triple hierarchical variational inequality problem (THVIP).

Problem 1.2 Let $M, N$ be two positive integers. Assume that

(i) $F: H \rightarrow H$ is $\kappa$-Lipschitzian and $\eta$-strongly monotone with positive constants $\kappa, \eta>0$ such that $0<\gamma \leq \tau$ and $0<\mu<\frac{2 \eta}{\kappa^{2}}$ where $\tau=1-\sqrt{1-\mu\left(2 \eta-\mu \kappa^{2}\right)}$;

(ii) for each $k \in\{1,2, \ldots, M\}, \Theta_{k}: C \times C \rightarrow \mathbb{R}$ satisfies conditions (A1)-(A4) and $\varphi_{k}: C \rightarrow \mathbb{R} \cup\{+\infty\}$ is a proper lower semicontinuous and convex function with restriction (B1) or (B2) (conditions (A1)-(A4) and (B1)-(B2) are given in the next section);

(iii) for each $k \in\{1,2, \ldots, M\}$ and $i \in\{1,2, \ldots, N\}, R_{i}: C \rightarrow 2^{H}$ is a maximal monotone mapping, and $A_{k}: H \rightarrow H$ and $B_{i}: C \rightarrow H$ are $\mu_{k}$-inverse strongly monotone and $\eta_{i}$-inverse strongly monotone, respectively;

(iv) $T: H \rightarrow H$ is a $\xi$-strict pseudocontraction, $S: H \rightarrow H$ is a nonexpansive mapping and $V: H \rightarrow H$ is a $\rho$-contraction with coefficient $\rho \in[0,1)$;

(v) $\Omega:=\left(\bigcap_{k=1}^{M} \operatorname{GMEP}\left(\Theta_{k}, \varphi_{k}, A_{k}\right)\right) \cap\left(\bigcap_{i=1}^{N} \mathrm{I}\left(B_{i}, R_{i}\right)\right) \cap \operatorname{Fix}(T) \cap \Gamma \neq \emptyset$. 
Then the objective is to find $x^{*} \in \Xi$ such that

$$
\left\langle(\mu F-\gamma V) x^{*}, x-x^{*}\right\rangle \geq 0, \quad \forall x \in \Xi
$$

where $\Xi$ denotes the solution set of the hierarchical variational inequality problem (HVIP) of finding $z^{*} \in \Omega$ such that

$$
\left\langle(\mu F-\gamma S) z^{*}, z-z^{*}\right\rangle \geq 0, \quad \forall z \in \Omega
$$

By combining Korpelevich's extragradient method, the viscosity approximation method, the hybrid steepest-descent method, Mann's iteration method, and the gradient-projection method (GPM) with regularization, we introduce and analyze a hybrid multi-step extragradient algorithm with regularization in the setting of Hilbert spaces. It is proven that under appropriate assumptions, the proposed algorithm converges strongly to a unique solution of THVIP (1.6). The algorithm and convergence result of this paper extend and generalize several existing algorithms and results, respectively, in the literature.

\section{Preliminaries}

Throughout this paper, unless otherwise specified, we assume that $H$ is a real Hilbert space whose inner product and norm are denoted by $\langle\cdot, \cdot\rangle\rangle$ and $\|\cdot\|$, respectively. We write $x_{n} \rightarrow x$ (respectively, $x_{n} \rightarrow x$ ) to indicate that the sequence $\left\{x_{n}\right\}$ converges (respectively, weakly) to $x$. Moreover, we use $\omega_{w}\left(x_{n}\right)$ to denote the weak $\omega$-limit set of the sequence $\left\{x_{n}\right\}$, that is,

$$
\omega_{w}\left(x_{n}\right):=\left\{x \in H: x_{n_{i}} \rightarrow x \text { for some subsequence }\left\{x_{n_{i}}\right\} \text { of }\left\{x_{n}\right\}\right\} .
$$

Definition 2.1 A mapping $T: H \rightarrow H$ is said to be

(a) nonexpansive if

$$
\|T x-T y\| \leq\|x-y\|, \quad \forall x, y \in H
$$

(b) firmly nonexpansive if $2 T-I$ is nonexpansive, or equivalently, if $T$ is 1-inverse strongly monotone (1-ism),

$$
\langle x-y, T x-T y\rangle \geq\|T x-T y\|^{2}, \quad \forall x, y \in H
$$

alternatively, $T$ is firmly nonexpansive if and only if $T$ can be expressed as

$$
T=\frac{1}{2}(I+S)
$$

where $S: H \rightarrow H$ is nonexpansive; projections are firmly nonexpansive.

It can easily be seen that if $T$ is nonexpansive, then $I-T$ is monotone.

Definition 2.2 A mapping $T: H \rightarrow H$ is said to be an averaged mapping if it can be written as the average of the identity $I$ and a nonexpansive mapping, that is,

$$
T \equiv(1-\alpha) I+\alpha S,
$$


where $\alpha \in(0,1)$ and $S: H \rightarrow H$ is nonexpansive. More precisely, when the last equality holds, we say that $T$ is $\alpha$-averaged. Thus firmly nonexpansive mappings (in particular, projections) are $\frac{1}{2}$-averaged mappings.

Proposition 2.1 [31] Let $T: H \rightarrow H$ be a given mapping.

(a) $T$ is nonexpansive if and only if the complement $I-T$ is $\frac{1}{2}$-ism.

(b) If $T$ is $v$-ism, then for $\gamma>0, \gamma T$ is $\frac{v}{\gamma}$-ism.

(c) $T$ is averaged if and only if the complement $I-T$ is $v$-ism for some $v>1 / 2$. Indeed, for $\alpha \in(0,1), T$ is $\alpha$-averaged if and only if $I-T$ is $\frac{1}{2 \alpha}$-ism.

Proposition 2.2 [31,32] Let $S, T, V: H \rightarrow H$ be given operators.

(a) If $T=(1-\alpha) S+\alpha V$ for some $\alpha \in(0,1)$ and if $S$ is averaged and $V$ is nonexpansive, then $T$ is averaged.

(b) $T$ is firmly nonexpansive if and only if the complement $I-T$ is firmly nonexpansive.

(c) If $T=(1-\alpha) S+\alpha V$ for some $\alpha \in(0,1)$ and if $S$ is firmly nonexpansive and $V$ is nonexpansive, then $T$ is averaged.

(d) The composite of finitely many averaged mappings is averaged, that is, if each of the mappings $\left\{T_{i}\right\}_{i=1}^{N}$ is averaged, then so is the composite $T_{1} \cdots T_{N}$. In particular, if $T_{1}$ is $\alpha_{1}$-averaged and $T_{2}$ is $\alpha_{2}$-averaged, where $\alpha_{1}, \alpha_{2} \in(0,1)$, then the composite $T_{1} T_{2}$ is $\alpha$-averaged, where $\alpha=\alpha_{1}+\alpha_{2}-\alpha_{1} \alpha_{2}$.

(e) If the mappings $\left\{T_{i}\right\}_{i=1}^{N}$ are averaged and have a common fixed point, then

$$
\bigcap_{i=1}^{N} \operatorname{Fix}\left(T_{i}\right)=\operatorname{Fix}\left(T_{1} \cdots T_{N}\right)
$$

The notation $\operatorname{Fix}(T)$ denotes the set of all fixed points of the mapping $T$, that is, $\operatorname{Fix}(T)=\{x \in H: T x=x\}$.

A mapping $T: C \rightarrow C$ is said to be $\xi$-strictly pseudocontractive if there exists $\xi \in[0,1)$ such that

$$
\|T x-T y\|^{2} \leq\|x-y\|^{2}+\xi\|(I-T) x-(I-T) y\|^{2}, \quad \forall x, y \in C .
$$

In this case, we also say that $T$ is a $\xi$-strict pseudocontraction. We denote by $\operatorname{Fix}(S)$ the set of fixed points of $S$. In particular, if $\xi=0, T$ is a nonexpansive mapping.

It is clear that, in a real Hilbert space $H, T: C \rightarrow C$ is $\xi$-strictly pseudocontractive if and only if the following inequality holds:

$$
\langle T x-T y, x-y\rangle \leq\|x-y\|^{2}-\frac{1-\xi}{2}\|(I-T) x-(I-T) y\|^{2}, \quad \forall x, y \in C .
$$

This immediately implies that if $T$ is a $\xi$-strictly pseudocontractive mapping, then $I-T$ is $\frac{1-\xi}{2}$-inverse strongly monotone; for further details, we refer to [33] and the references therein. It is well known that the class of strict pseudocontractions strictly includes the class of nonexpansive mappings and that the class of pseudocontractions strictly includes the class of strict pseudocontractions.

Lemma 2.1 [33, Proposition 2.1] Let C be a nonempty closed convex subset of a real Hilbert space $H$ and $T: C \rightarrow C$ be a mapping. 
(a) If $T$ is a $\xi$-strictly pseudocontractive mapping, then $T$ satisfies the Lipschitzian condition

$$
\|T x-T y\| \leq \frac{1+\xi}{1-\xi}\|x-y\|, \quad \forall x, y \in C .
$$

(b) If $T$ is a $\xi$-strictly pseudocontractive mapping, then the mapping $I-T$ is semiclosed at 0 , that is, if $\left\{x_{n}\right\}$ is a sequence in $C$ such that $x_{n} \rightarrow \tilde{x}$ and $(I-T) x_{n} \rightarrow 0$, then $(I-T) \tilde{x}=0$.

(c) If $T$ is $\xi$-(quasi-)strict pseudocontraction, then the fixed point set $\operatorname{Fix}(T)$ of $T$ is closed and convex so that the projection $P_{\mathrm{Fix}(T)}$ is well defined.

Lemma 2.2 [34] Let $C$ be a nonempty closed convex subset of a real Hilbert space H. Let $T: C \rightarrow C$ be a $\xi$-strictly pseudocontractive mapping. Let $\gamma$ and $\delta$ be two nonnegative real numbers such that $(\gamma+\delta) \xi \leq \gamma$. Then

$$
\|\gamma(x-y)+\delta(T x-T y)\| \leq(\gamma+\delta)\|x-y\|, \quad \forall x, y \in C
$$

Lemma 2.3 (Demiclosedness principle) Let $C$ be a nonempty closed convex subset of a real Hilbert space $H$. Let $S$ be a nonexpansive self-mapping on $C$ with $\operatorname{Fix}(S) \neq \emptyset$. Then $I-S$ is demiclosed. That is, whenever $\left\{x_{n}\right\}$ is a sequence in $C$ weakly converging to some $x \in C$ and the sequence $\left\{(I-S) x_{n}\right\}$ strongly converges to some $y$, it follows that $(I-S) x=y$, where $I$ is the identity operator of $H$.

Definition 2.3 A nonlinear operator $T$ with the domain $D(T) \subset H$ and the range $R(T) \subset$ $H$ is said to be

(a) monotone if

$$
\langle T x-T y, x-y\rangle \geq 0, \quad \forall x, y \in D(T)
$$

(b) $\beta$-strongly monotone if there exists a constant $\beta>0$ such that

$$
\langle T x-T y, x-y\rangle \geq \eta\|x-y\|^{2}, \quad \forall x, y \in D(T)
$$

(c) $v$-inverse strongly monotone if there exists a constant $v>0$ such that

$$
\langle T x-T y, x-y\rangle \geq v\|T x-T y\|^{2}, \quad \forall x, y \in D(T) .
$$

It is easy to see that the projection $P_{C}$ is 1-inverse strongly monotone. Inverse strongly monotone (also referred to as co-coercive) operators have been applied widely in solving practical problems in various fields, for instance, in traffic assignment problems; see, for example, [35]. It is obvious that if $T$ is $v$-inverse strongly monotone, then $T$ is monotone and $\frac{1}{v}$-Lipschitz continuous. Moreover, we also have, for all $u, v \in D(T)$ and $\lambda>0$,

$$
\begin{aligned}
\|(I-\lambda T) u-(I-\lambda T) v\|^{2} & =\|(u-v)-\lambda(T u-T v)\|^{2} \\
& =\|u-v\|^{2}-2 \lambda\langle T u-T v, u-v\rangle+\lambda^{2}\|T u-T v\|^{2} \\
& \leq\|u-v\|^{2}+\lambda(\lambda-2 v)\|T u-T v\|^{2} .
\end{aligned}
$$

So, if $\lambda \leq 2 v$, then $I-\lambda T$ is a nonexpansive mapping. 
The metric (or nearest point) projection from $H$ onto $C$ is the mapping $P_{C}: H \rightarrow C$ which assigns to each point $x \in H$ the unique point $P_{C} x \in C$ satisfying the property

$$
\left\|x-P_{C} x\right\|=\inf _{y \in C}\|x-y\|=: d(x, C) .
$$

Some important properties of projections are gathered in the following proposition.

Proposition 2.3 For given $x \in H$ and $z \in C$ :

(a) $z=P_{C} x \Leftrightarrow\langle x-z, y-z\rangle \leq 0, \forall y \in C$;

(b) $z=P_{C} x \Leftrightarrow\|x-z\|^{2} \leq\|x-y\|^{2}-\|y-z\|^{2}, \forall y \in C$;

(c) $\left\langle P_{C} x-P_{C} y, x-y\right\rangle \geq\left\|P_{C} x-P_{C} y\right\|^{2}, \forall y \in H$.

Consequently, $P_{C}$ is nonexpansive and monotone.

Let $\lambda$ be a number in $(0,1]$ and let $\mu>0$. Associating with a nonexpansive mapping $T: C \rightarrow H$, we define the mapping $T^{\lambda}: C \rightarrow H$ by

$$
T^{\lambda} x:=T x-\lambda \mu F(T x), \quad \forall x \in C
$$

where $F: H \rightarrow H$ is an operator such that, for some positive constants $\kappa, \eta>0, F$ is $\kappa$ Lipschitzian and $\eta$-strongly monotone on $H$, that is, $F$ satisfies the conditions:

$$
\|F x-F y\| \leq \kappa\|x-y\| \quad \text { and } \quad\langle F x-F y, x-y\rangle \geq \eta\|x-y\|^{2}
$$

for all $x, y \in H$.

Lemma 2.4 [36, Lemma 3.1] $T^{\lambda}$ is a contraction provided $0<\mu<\frac{2 \eta}{\kappa^{2}}$, that is,

$$
\left\|T^{\lambda} x-T^{\lambda} y\right\| \leq(1-\lambda \tau)\|x-y\|, \quad \forall x, y \in C,
$$

where $\tau=1-\sqrt{1-\mu\left(2 \eta-\mu \kappa^{2}\right)} \in(0,1]$.

Lemma 2.5 Let $A: C \rightarrow H$ be a monotone mapping. In the context of the variational inequality problem the characterization of the projection (see Proposition 2.3(a)) implies

$$
u \in \operatorname{VI}(C, A) \quad \Leftrightarrow \quad u=P_{C}(u-\lambda A u), \quad \forall \lambda>0 .
$$

Let $C$ be a nonempty closed convex subset of $H$ and $\Theta: C \times C \rightarrow \mathbb{R}$ satisfy the following conditions.

(A1) $\Theta(x, x)=0, \forall x \in C$;

(A2) $\Theta$ is monotone, that is, $\Theta(x, y)+\Theta(y, x) \leq 0, \forall x, y \in C$;

(A3) $\Theta$ is upper-hemicontinuous, that is, $\forall x, y, z \in C$,

$$
\limsup _{t \rightarrow 0^{+}} \Theta(t z+(1-t) x, y) \leq \Theta(x, y) ;
$$

(A4) $\Theta(x, \cdot)$ is convex and lower semicontinuous, for each $x \in C$.

Let $\varphi: C \rightarrow \mathbb{R}$ be a lower semicontinuous and convex function satisfying either (B1) or (B2), where 
(B1) for each $x \in H$ and $r>0$, there exist a bounded subset $D_{x} \subset C$ and $y_{x} \in C$ such that, for any $z \in C \backslash D_{x}$,

$$
\Theta\left(z, y_{x}\right)+\varphi\left(y_{x}\right)-\varphi(z)+\frac{1}{r}\left\langle y_{x}-z, z-x\right\rangle<0
$$

(B2) $C$ is a bounded set.

Given a positive number $r>0$. Let $T_{r}^{(\Theta, \varphi)}: H \rightarrow C$ be the solution set of the auxiliary mixed equilibrium problem, that is, for each $x \in H$,

$$
T_{r}^{(\Theta, \varphi)}(x):=\left\{y \in C: \Theta(y, z)+\varphi(z)-\varphi(y)+\frac{1}{r}\langle y-x, z-y\rangle \geq 0, \forall z \in C\right\} .
$$

Next we list some elementary conclusions for the MEP.

Proposition 2.4 [37] Assume that $\Theta: C \times C \rightarrow \mathbb{R}$ satisfies (A1)-(A4) and let $\varphi: C \rightarrow \mathbf{R}$ be a proper lower semicontinuous and convex function. Assume that either (B1) or (B2) holds. For $r>0$ and $x \in H$, define a mapping $T_{r}^{(\Theta, \varphi)}: H \rightarrow C$ as follows:

$$
T_{r}^{(\Theta, \varphi)}(x):=\left\{z \in C: \Theta(z, y)+\varphi(y)-\varphi(z)+\frac{1}{r}\langle y-z, z-x\rangle \geq 0, \forall y \in C\right\}
$$

for all $x \in H$. Then

(i) for each $x \in H, T_{r}^{(\Theta, \varphi)}(x)$ is nonempty and single-valued;

(ii) $T_{r}^{(\Theta, \varphi)}$ is firmly nonexpansive, that is, for any $x, y \in H$,

$$
\left\|T_{r}^{(\Theta, \varphi)} x-T_{r}^{(\Theta, \varphi)} y\right\|^{2} \leq\left\langle T_{r}^{(\Theta, \varphi)} x-T_{r}^{(\Theta, \varphi)} y, x-y\right\rangle
$$

(iii) $\operatorname{Fix}\left(T_{r}^{(\Theta, \varphi)}\right)=\operatorname{MEP}(\Theta, \varphi)$;

(iv) $\operatorname{MEP}(\Theta, \varphi)$ is closed and convex;

(v) $\left\|T_{s}^{(\Theta, \varphi)} x-T_{t}^{(\Theta, \varphi)} x\right\|^{2} \leq \frac{s-t}{s}\left\langle T_{s}^{(\Theta, \varphi)} x-T_{t}^{(\Theta, \varphi)} x, T_{s}^{(\Theta, \varphi)} x-x\right\rangle$, for all $s, t>0$ and $x \in H$.

We need some facts and tools in a real Hilbert space $H$ which are listed as lemmas below.

Lemma 2.6 Let $X$ be a real inner product space. Then we have the following inequality:

$$
\|x+y\|^{2} \leq\|x\|^{2}+2\langle y, x+y\rangle, \quad \forall x, y \in X
$$

Lemma 2.7 Let $H$ be a real Hilbert space. Then the following hold:

(a) $\|x-y\|^{2}=\|x\|^{2}-\|y\|^{2}-2\langle x-y, y\rangle$, for all $x, y \in H$;

(b) $\|\lambda x+\mu y\|^{2}=\lambda\|x\|^{2}+\mu\|y\|^{2}-\lambda \mu\|x-y\|^{2}$, for all $x, y \in H$ and $\lambda, \mu \in[0,1]$ with $\lambda+\mu=1$

(c) if $\left\{x_{n}\right\}$ is a sequence in $H$ such that $x_{n} \rightarrow x$, it follows that

$$
\limsup _{n \rightarrow \infty}\left\|x_{n}-y\right\|^{2}=\limsup _{n \rightarrow \infty}\left\|x_{n}-x\right\|^{2}+\|x-y\|^{2}, \quad \forall y \in H
$$

Lemma 2.8 [38] Let $\left\{a_{n}\right\}$ be a sequence of nonnegative real numbers satisfying the property

$$
a_{n+1} \leq\left(1-s_{n}\right) a_{n}+s_{n} b_{n}+t_{n}, \quad \forall n \geq 1
$$


where $\left\{s_{n}\right\} \subset(0,1]$ and $\left\{b_{n}\right\}$ are such that:

(i) $\sum_{n=1}^{\infty} s_{n}=\infty$;

(ii) either $\limsup _{n \rightarrow \infty} b_{n} \leq 0$ or $\sum_{n=0}^{\infty}\left|s_{n} b_{n}\right|<\infty$;

(iii) $\sum_{n=1}^{\infty} t_{n}<\infty$ where $t_{n} \geq 0$, for all $n \geq 1$.

Then $\lim _{n \rightarrow \infty} a_{n}=0$.

Recall that a set-valued mapping $T: D(T) \subset H \rightarrow 2^{H}$ is called monotone if, for all $x, y \in D(T), f \in T x$, and $g \in T y$ imply $\langle f-g, x-y\rangle \geq 0$. A set-valued mapping $T$ is called maximal monotone if $T$ is monotone and $(I+\lambda T) D(T)=H$, for each $\lambda>0$, where $I$ is the identity mapping of $H$. We denote by $G(T)$ the graph of $T$. It is well known that a monotone mapping $T$ is maximal if and only if, for $(x, f) \in H \times H,\langle f-g, x-y\rangle \geq 0$ for every $(y, g) \in G(T)$ implies $f \in T x$.

Next we provide an example to illustrate the concept of maximal monotone mapping.

Let $A: C \rightarrow H$ be a monotone, $k$-Lipschitz-continuous mapping and let $N_{C} v$ be the normal cone to $C$ at $v \in C$, that is,

$$
N_{C} v=\{u \in H:\langle v-p, u\rangle \geq 0, \forall p \in C\} .
$$

Define

$$
\widetilde{T} v= \begin{cases}A v+N_{C} v, & \text { if } v \in C, \\ \emptyset, & \text { if } v \notin C .\end{cases}
$$

Then $\widetilde{T}$ is maximal monotone (see [16]) such that

$$
0 \in \widetilde{T} v \quad \Leftrightarrow \quad v \in \operatorname{VI}(C, A) .
$$

Let $R: D(R) \subset H \rightarrow 2^{H}$ be a maximal monotone mapping. Let $\lambda, \mu>0$ be two positive numbers.

Lemma 2.9 [39] We have the resolvent identity

$$
J_{R, \lambda} x=J_{R, \mu}\left(\frac{\mu}{\lambda} x+\left(1-\frac{\mu}{\lambda}\right) J_{R, \lambda} x\right), \quad \forall x \in H
$$

Remark 2.1 For $\lambda, \mu>0$, we have the following relation:

$$
\left\|J_{R, \lambda} x-J_{R, \mu} y\right\| \leq\|x-y\|+|\lambda-\mu|\left(\frac{1}{\lambda}\left\|J_{R, \lambda} x-y\right\|+\frac{1}{\mu}\left\|x-J_{R, \mu} y\right\|\right), \quad \forall x, y \in H .
$$

The following property for the resolvent operator $J_{R, \lambda}: H \rightarrow \overline{D(R)}$ was considered in $[17,18]$.

Lemma 2.10 $J_{R, \lambda}$ is single-valued and firmly nonexpansive, that is,

$$
\left\langle J_{R, \lambda} x-J_{R, \lambda} y, x-y\right\rangle \geq\left\|J_{R, \lambda} x-J_{R, \lambda} y\right\|^{2}, \quad \forall x, y \in H .
$$

Consequently, $J_{R, \lambda}$ is nonexpansive and monotone. 
Lemma 2.11 [20] Let $R$ be a maximal monotone mapping with $D(R)=C$. Then, for any given $\lambda>0, u \in C$ is a solution of problem (1.6) if and only if $u \in C$ satisfies

$$
u=J_{R, \lambda}(u-\lambda B u) .
$$

Lemma 2.12 [18] Let $R$ be a maximal monotone mapping with $D(R)=C$ and let $B: C \rightarrow H$ be a strongly monotone, continuous and single-valued mapping. Then, for each $z \in H$, the equation $z \in(B+\lambda R) x$ has a unique solution $x_{\lambda}$ for $\lambda>0$.

Lemma 2.13 [20] Let $R$ be a maximal monotone mapping with $D(R)=C$ and $B: C \rightarrow H$ be a monotone, continuous and single-valued mapping. Then $(I+\lambda(R+B)) C=H$, for each $\lambda>0$. In this case, $R+B$ is maximal monotone.

\section{Algorithms and convergence results}

Let $H$ be a real Hilbert space and $f: H \rightarrow \mathbb{R}$ be a function. Then the minimization problem

$$
\min _{x \in C} f(x):=\frac{1}{2}\left\|A x-P_{Q} A x\right\|^{2}
$$

is ill-posed. Xu [40] considered the following Tikhonov's regularization problem:

$$
\min _{x \in C} f_{\alpha}(x):=\frac{1}{2}\left\|A x-P_{Q} A x\right\|^{2}+\frac{1}{2} \alpha\|x\|^{2},
$$

where $\alpha>0$ is the regularization parameter. It is clear that the gradient

$$
\nabla f_{\alpha}=\nabla f+\alpha I=A^{*}\left(I-P_{Q}\right) A+\alpha I
$$

is $\left(\alpha+\|A\|^{2}\right)$-Lipschitz continuous.

Throughout the paper, unless otherwise specified, $M, N$ are positive integers and $C$ be a nonempty closed convex subset of a real Hilbert space $H$.

Algorithm 3.1 The notations and symbols are the same as in Problem 1.2. Start with a given arbitrary $x_{0} \in H$, and compute a sequence $\left\{x_{n}\right\}$ by

$$
\left\{\begin{array}{l}
u_{n}=T_{r_{M, n}}^{\left(\Theta_{M}, \varphi_{M}\right)}\left(I-r_{M, n} A_{M}\right) T_{r_{M-1, n}}^{\left(\Theta_{M-1}, \varphi_{M-1}\right)}\left(I-r_{M-1, n} A_{M-1}\right) \cdots T_{r_{1, n}}^{\left(\Theta_{1}, \varphi_{1}\right)}\left(I-r_{1, n} A_{1}\right) x_{n}, \\
v_{n}=J_{R_{N}, \lambda_{N, n}}\left(I-\lambda_{N, n} B_{N}\right) J_{R_{N-1}, \lambda_{N-1, n}}\left(I-\lambda_{N-1, n} B_{N-1}\right) \cdots J_{R_{1}, \lambda_{1, n}}\left(I-\lambda_{1, n} B_{1}\right) u_{n}, \\
y_{n}=\beta_{n} x_{n}+\gamma_{n} P_{C}\left(I-\lambda_{n} \nabla f_{\alpha_{n}}\right) v_{n}+\sigma_{n} T P_{C}\left(I-\lambda_{n} \nabla f_{\alpha_{n}}\right) v_{n}, \\
x_{n+1}=\epsilon_{n} \gamma\left(\delta_{n} V x_{n}+\left(1-\delta_{n}\right) S x_{n}\right)+\left(I-\epsilon_{n} \mu F\right) y_{n}, \quad \forall n \geq 0
\end{array}\right.
$$

where $\nabla f_{\alpha_{n}}=\alpha_{n} I+\nabla f$.

The following result provides the strong convergence of the sequence generated by $\mathrm{Al}-$ gorithm 3.1.

Theorem 3.1 For each $k \in\{1,2, \ldots, M\}$, let $\Theta_{k}: C \times C \rightarrow \mathbb{R}$ be a bifunction satisfying conditions (A1)-(A4) and $\varphi_{k}: C \rightarrow \mathbb{R} \cup\{+\infty\}$ be a proper lower semicontinuous and convex function with restriction (B1) or (B2). For each $k \in\{1,2, \ldots, M\}$ and $i \in\{1,2, \ldots, N\}$, 
let $R_{i}: C \rightarrow 2^{H}$ be a maximal monotone mapping and let $A_{k}: H \rightarrow H$ and $B_{i}: C \rightarrow H$ be $\mu_{k}$-inverse strongly monotone and $\eta_{i}$-inverse strongly monotone, respectively. Let $T$ : $H \rightarrow H$ be a $\xi$-strictly pseudocontractive mapping, $S: H \rightarrow H$ be a nonexpansive mapping and $V: H \rightarrow H$ be a $\rho$-contraction with coefficient $\rho \in[0,1)$. Let $F: H \rightarrow H$ be $\kappa$ Lipschitzian and $\eta$-strongly monotone with positive constants $\kappa, \eta>0$ such that $0<\gamma \leq \tau$ and $0<\mu<\frac{2 \eta}{\kappa^{2}}$, where $\tau=1-\sqrt{1-\mu\left(2 \eta-\mu \kappa^{2}\right)}$. Assume that the solution set $\Xi$ of HVIP (1.7) is nonempty where $\Omega:=\left(\bigcap_{k=1}^{M} \operatorname{GMEP}\left(\Theta_{k}, \varphi_{k}, A_{k}\right)\right) \cap\left(\bigcap_{i=1}^{N} \mathrm{I}\left(B_{i}, R_{i}\right)\right) \cap \operatorname{Fix}(T) \cap \Gamma$. Let $\left\{\lambda_{n}\right\} \subset[a, b] \subset\left(0, \frac{2}{\|A\|^{2}}\right),\left\{\alpha_{n}\right\} \subset(0, \infty)$ with $\sum_{n=0}^{\infty} \alpha_{n}<\infty,\left\{\epsilon_{n}\right\},\left\{\delta_{n}\right\},\left\{\beta_{n}\right\},\left\{\gamma_{n}\right\},\left\{\sigma_{n}\right\} \subset(0,1)$ with $\beta_{n}+\gamma_{n}+\sigma_{n}=1$, and $\left\{\lambda_{i, n}\right\} \subset\left[a_{i}, b_{i}\right] \subset\left(0,2 \eta_{i}\right),\left\{r_{k, n}\right\} \subset\left[c_{k}, d_{k}\right] \subset\left(0,2 \mu_{k}\right)$ where $i \in\{1,2, \ldots, N\}$ and $k \in\{1,2, \ldots, M\}$. Suppose that

(C1) $\lim _{n \rightarrow \infty} \delta_{n}=0, \lim _{n \rightarrow \infty} \epsilon_{n}=0, \lim _{n \rightarrow \infty} \frac{\left|\epsilon_{n}-\epsilon_{n-1}\right|}{\delta_{n} \epsilon_{n}^{2} \epsilon_{n-1}}=0$ and $\sum_{n=0}^{\infty} \epsilon_{n} \delta_{n}=\infty$;

(C2) $\sum_{n=1}^{\infty}\left|\delta_{n}-\delta_{n-1}\right|<\infty$ or $\lim _{n \rightarrow \infty}\left|\delta_{n}-\delta_{n-1}\right| /\left(\delta_{n} \epsilon_{n}\right)=0$;

(C3) $\sum_{n=1}^{\infty} \frac{\left|\beta_{n}-\beta_{n-1}\right|}{\epsilon_{n}}<\infty$ or $\lim _{n \rightarrow \infty}\left|\beta_{n}-\beta_{n-1}\right| /\left(\delta_{n} \epsilon_{n}^{2}\right)=0$;

(C4) $\sum_{n=1}^{\infty} \frac{\left|\gamma_{n}-\gamma_{n-1}\right|}{\epsilon_{n}}<\infty$ or $\lim _{n \rightarrow \infty}\left|\gamma_{n}-\gamma_{n-1}\right| /\left(\delta_{n} \epsilon_{n}^{2}\right)=0$;

(C5) $\sum_{n=1}^{\infty} \frac{\left|\lambda_{n}-\lambda_{n-1}\right|}{\epsilon_{n}}<\infty$ or $\lim _{n \rightarrow \infty}\left|\lambda_{n}-\lambda_{n-1}\right| /\left(\delta_{n} \epsilon_{n}^{2}\right)=0$;

(C6) $\sum_{n=1}^{\infty} \frac{\left|\lambda_{n} \alpha_{n}-\lambda_{n-1} \alpha_{n-1}\right|}{\epsilon_{n}}<\infty$ or $\lim _{n \rightarrow \infty}\left|\lambda_{n} \alpha_{n}-\lambda_{n-1} \alpha_{n-1}\right| /\left(\delta_{n} \epsilon_{n}^{2}\right)=0$;

(C7) $\left\{\beta_{n}\right\} \subset[c, d] \subset(0,1),\left(\gamma_{n}+\sigma_{n}\right) \xi \leq \gamma_{n}$ and $\liminf _{n \rightarrow \infty} \sigma_{n}>0$;

(C8) for each $i=1,2, \ldots, N, \sum_{n=1}^{\infty} \frac{\left|\lambda_{i, n}-\lambda_{i, n-1}\right|}{\epsilon_{n}}<\infty$ or $\lim _{n \rightarrow \infty}\left|\lambda_{i, n}-\lambda_{i, n-1}\right| /\left(\delta_{n} \epsilon_{n}^{2}\right)=0$;

(C9) for each $k=1,2, \ldots, M, \sum_{n=1}^{\infty} \frac{\left|r_{k, n}-r_{k, n-1}\right|}{\epsilon_{n}}<\infty$ or $\lim _{n \rightarrow \infty}\left|r_{k, n}-r_{k, n-1}\right| /\left(\delta_{n} \epsilon_{n}^{2}\right)=0$;

(C10) there exist positive constants $\theta, \bar{k}>0$ such that $\lim _{n \rightarrow \infty} \epsilon_{n}^{1 / \theta} / \delta_{n}=0$ and $\left\|x_{n}-T x_{n}\right\| \geq \bar{k}\left[d\left(x_{n}, \Omega\right)\right]^{\theta}, \forall x \in C$ for sufficiently large $n \geq 0$.

If $\left\{x_{n}\right\}$ is a sequence generated by Algorithm 3.1 and $\left\{S x_{n}\right\}$ is bounded, then

(a) $\left\|x_{n+1}-x_{n}\right\|=o\left(\epsilon_{n}\right)$;

(b) $\omega_{w}\left(x_{n}\right) \subset \Omega$;

(c) $\left\{x_{n}\right\}$ converges strongly to a point $x^{*} \in \Omega$ provided $\left\|x_{n}-y_{n}\right\|+\alpha_{n}=o\left(\epsilon_{n}^{2}\right)$, which is the unique solution of Problem 1.2.

Proof First of all, taking into account $\Xi \neq \emptyset$, we know that $\Omega \neq \emptyset$. Observe that

$$
\begin{aligned}
\mu \eta \geq \tau & \Leftrightarrow \mu \eta \geq 1-\sqrt{1-\mu\left(2 \eta-\mu \kappa^{2}\right)} \\
& \Leftrightarrow \sqrt{1-\mu\left(2 \eta-\mu \kappa^{2}\right)} \geq 1-\mu \eta \\
& \Leftrightarrow 1-2 \mu \eta+\mu^{2} \kappa^{2} \geq 1-2 \mu \eta+\mu^{2} \eta^{2} \\
& \Leftrightarrow \kappa^{2} \geq \eta^{2} \\
& \Leftrightarrow \kappa \geq \eta
\end{aligned}
$$

and

$$
\begin{aligned}
\langle(\mu F-\gamma V) x-(\mu F-\gamma V) y, x-y\rangle & =\mu\langle F x-F y, x-y\rangle-\gamma\langle V x-V y, x-y\rangle \\
& \geq \mu \eta\|x-y\|^{2}-\gamma \rho\|x-y\|^{2} \\
& =(\mu \eta-\gamma \rho)\|x-y\|^{2}, \quad \forall x, y \in H .
\end{aligned}
$$

Since $\tau \geq \gamma>0$ and $\kappa \geq \eta$, we deduce that $\mu \eta \geq \tau \geq \gamma>\gamma \rho$ and hence the mapping $\mu F-\gamma V$ is $(\mu \eta-\gamma \rho)$-strongly monotone. Moreover, it is clear that the mapping $\mu F-\gamma V$ 
is $(\mu \kappa+\gamma \rho)$-Lipschitzian. Thus, there exists a unique solution $x^{*}$ in $\Xi$ to the VIP

$$
\left\langle(\mu F-\gamma V) x^{*}, p-x^{*}\right\rangle \geq 0, \quad \forall p \in \Xi
$$

that is, $\left\{x^{*}\right\}=\operatorname{VI}(\Xi, \mu F-\gamma V)$. Now, we put

$$
\Delta_{n}^{k}=T_{r_{k, n}}^{\left(\Theta_{k}, \varphi_{k}\right)}\left(I-r_{k, n} A_{k}\right) T_{r_{k-1, n}}^{\left(\Theta_{k-1}, \varphi_{k-1}\right)}\left(I-r_{k-1, n} A_{k-1}\right) \cdots T_{r_{1, n}}^{\left(\Theta_{1}, \varphi_{1}\right)}\left(I-r_{1, n} A_{1}\right) x_{n}
$$

for all $k \in\{1,2, \ldots, M\}$ and $n \geq 0$,

$$
\Lambda_{n}^{i}=J_{R_{i}, \lambda_{i, n}}\left(I-\lambda_{i, n} B_{i}\right) J_{R_{i-1}, \lambda_{i-1, n}}\left(I-\lambda_{i-1, n} B_{i-1}\right) \cdots J_{R_{1}, \lambda_{1, n}}\left(I-\lambda_{1, n} B_{1}\right)
$$

for all $i \in\{1,2, \ldots, N\}, \Delta_{n}^{0}=I$, and $\Lambda_{n}^{0}=I$, where $I$ is the identity mapping on $H$. Then we have $u_{n}=\Delta_{n}^{M} x_{n}$ and $v_{n}=\Lambda_{n}^{N} u_{n}$.

Now, we show that $P_{C}\left(I-\lambda \nabla f_{\alpha}\right)$ is $\zeta$-averaged, for each $\lambda \in\left(0, \frac{2}{\alpha+\|A\|^{2}}\right)$, where

$$
\zeta=\frac{2+\lambda\left(\alpha+\|A\|^{2}\right)}{4} \in(0,1)
$$

Indeed, it is easy to see that $\nabla f=A^{*}\left(I-P_{Q}\right) A$ is $\frac{1}{\|A\|^{2}}$-ism, that is,

$$
\langle\nabla f(x)-\nabla f(y), x-y\rangle \geq \frac{1}{\|A\|^{2}}\|\nabla f(x)-\nabla f(y)\|^{2} .
$$

Observe that

$$
\begin{aligned}
(\alpha+ & \left.\|A\|^{2}\right)\left\langle\nabla f_{\alpha}(x)-\nabla f_{\alpha}(y), x-y\right\rangle \\
= & \left(\alpha+\|A\|^{2}\right)\left[\alpha\|x-y\|^{2}+\langle\nabla f(x)-\nabla f(y), x-y\rangle\right] \\
= & \alpha^{2}\|x-y\|^{2}+\alpha\langle\nabla f(x)-\nabla f(y), x-y\rangle \\
& \quad+\alpha\|A\|^{2}\|x-y\|^{2}+\|A\|^{2}\langle\nabla f(x)-\nabla f(y), x-y\rangle \\
\geq & \alpha^{2}\|x-y\|^{2}+2 \alpha\langle\nabla f(x)-\nabla f(y), x-y\rangle+\|\nabla f(x)-\nabla f(y)\|^{2} \\
= & \|\alpha(x-y)+\nabla f(x)-\nabla f(y)\|^{2} \\
= & \left\|\nabla f_{\alpha}(x)-\nabla f_{\alpha}(y)\right\|^{2} .
\end{aligned}
$$

Hence, it follows that $\nabla f_{\alpha}=\alpha I+A^{*}\left(I-P_{Q}\right) A$ is $\frac{1}{\alpha+\|A\|^{2}}$-ism. Thus, by Proposition 2.1(b), $\lambda \nabla f_{\alpha}$ is $\frac{1}{\lambda\left(\alpha+\|A\|^{2}\right)}$-ism. From Proposition 2.1(c), the complement $I-\lambda \nabla f_{\alpha}$ is $\frac{\lambda\left(\alpha+\|A\|^{2}\right)}{2}$ averaged. Therefore, noting that $P_{C}$ is $\frac{1}{2}$-averaged and utilizing Proposition 2.2(d), we see that, for each $\lambda \in\left(0, \frac{2}{\alpha+\|A\|^{2}}\right), P_{C}\left(I-\lambda \nabla f_{\alpha}\right)$ is $\zeta$-averaged with

$$
\zeta=\frac{1}{2}+\frac{\lambda\left(\alpha+\|A\|^{2}\right)}{2}-\frac{1}{2} \cdot \frac{\lambda\left(\alpha+\|A\|^{2}\right)}{2}=\frac{2+\lambda\left(\alpha+\|A\|^{2}\right)}{4} \in(0,1) .
$$

This shows that $P_{C}\left(I-\lambda \nabla f_{\alpha}\right)$ is nonexpansive. Taking into account that $\left\{\lambda_{n}\right\} \subset[a, b] \subset$ $\left(0, \frac{2}{\|A\|^{2}}\right)$ and $\alpha_{n} \rightarrow 0$, we get

$$
\limsup _{n \rightarrow \infty} \frac{2+\lambda_{n}\left(\alpha_{n}+\|A\|^{2}\right)}{4} \leq \frac{2+b\|A\|^{2}}{4}<1 .
$$


Without loss of generality, we may assume that $\zeta_{n}:=\frac{2+\lambda_{n}\left(\alpha_{n}+\|A\|^{2}\right)}{4}<1$, for each $n \geq 0$. So, $P_{C}\left(I-\lambda_{n} \nabla f_{\alpha_{n}}\right)$ is nonexpansive, for each $n \geq 0$. Similarly, since

$$
\limsup _{n \rightarrow \infty} \frac{\lambda_{n}\left(\alpha_{n}+\|A\|^{2}\right)}{2} \leq \frac{b\|A\|^{2}}{2}<1
$$

it may be confirmed that $I-\lambda_{n} \nabla f_{\alpha_{n}}$ is nonexpansive, for each $n \geq 0$.

We divide the rest of the proof into several steps.

Step 1 . We prove that $\left\{x_{n}\right\}$ is bounded.

Indeed, take a fixed $p \in \Omega$ arbitrarily. Utilizing (2.1) and Proposition 2.4(b), we have

$$
\begin{aligned}
\left\|u_{n}-p\right\|= & \left\|T_{r_{M, n}}^{\left(\Theta_{M}, \varphi_{M}\right)}\left(I-r_{M, n} B_{M}\right) \Delta_{n}^{M-1} x_{n}-T_{r_{M, n}}^{\left(\Theta_{M}, \varphi_{M}\right)}\left(I-r_{M, n} B_{M}\right) \Delta_{n}^{M-1} p\right\| \\
\leq & \left\|\left(I-r_{M, n} B_{M}\right) \Delta_{n}^{M-1} x_{n}-\left(I-r_{M, n} B_{M}\right) \Delta_{n}^{M-1} p\right\| \\
\leq & \left\|\Delta_{n}^{M-1} x_{n}-\Delta_{n}^{M-1} p\right\| \\
& \vdots \\
\leq & \left\|\Delta_{n}^{0} x_{n}-\Delta_{n}^{0} p\right\| \\
= & \left\|x_{n}-p\right\| .
\end{aligned}
$$

Utilizing (2.1) and Lemma 2.10, we have

$$
\begin{aligned}
\left\|v_{n}-p\right\|= & \left\|J_{R_{N}, \lambda_{N, n}}\left(I-\lambda_{N, n} A_{N}\right) \Lambda_{n}^{N-1} u_{n}-J_{R_{N}, \lambda_{N, n}}\left(I-\lambda_{N, n} A_{N}\right) \Lambda_{n}^{N-1} p\right\| \\
\leq & \left\|\left(I-\lambda_{N, n} A_{N}\right) \Lambda_{n}^{N-1} u_{n}-\left(I-\lambda_{N, n} A_{N}\right) \Lambda_{n}^{N-1} p\right\| \\
\leq & \left\|\Lambda_{n}^{N-1} u_{n}-\Lambda_{n}^{N-1} p\right\| \\
& \vdots \\
\leq & \left\|\Lambda_{n}^{0} u_{n}-\Lambda_{n}^{0} p\right\| \\
= & \left\|u_{n}-p\right\| .
\end{aligned}
$$

Combining (3.2) and (3.3), we have

$$
\left\|v_{n}-p\right\| \leq\left\|x_{n}-p\right\|
$$

For simplicity, put $t_{n}=P_{C}\left(I-\lambda_{n} \nabla f_{\alpha_{n}}\right) v_{n}$, for each $n \geq 0$. Note that $P_{C}(I-\lambda \nabla f) p=p$ for $\lambda \in\left(0, \frac{2}{\|A\|^{2}}\right)$. Hence, from (3.4), it follows that

$$
\begin{aligned}
\left\|t_{n}-p\right\|= & \left\|P_{C}\left(I-\lambda_{n} \nabla f_{\alpha_{n}}\right) v_{n}-P_{C}\left(I-\lambda_{n} \nabla f\right) p\right\| \\
\leq & \left\|P_{C}\left(I-\lambda_{n} \nabla f_{\alpha_{n}}\right) v_{n}-P_{C}\left(I-\lambda_{n} \nabla f_{\alpha_{n}}\right) p\right\| \\
& \quad\left\|P_{C}\left(I-\lambda_{n} \nabla f_{\alpha_{n}}\right) p-P_{C}\left(I-\lambda_{n} \nabla f\right) p\right\| \\
\leq & \left\|v_{n}-p\right\|+\left\|\left(I-\lambda_{n} \nabla f_{\alpha_{n}}\right) p-\left(I-\lambda_{n} \nabla f\right) p\right\| \\
= & \left\|v_{n}-p\right\|+\lambda_{n} \alpha_{n}\|p\| \\
\leq & \left\|x_{n}-p\right\|+\lambda_{n} \alpha_{n}\|p\| .
\end{aligned}
$$


Since $T$ is a $\xi$-strictly pseudocontractive mapping and $\left(\gamma_{n}+\sigma_{n}\right) \xi \leq \gamma_{n}$, for all $n \geq 0$, by Lemma 2.2, we obtain from (3.1) and (3.5) that

$$
\begin{aligned}
\left\|y_{n}-p\right\| & =\left\|\beta_{n} x_{n}+\gamma_{n} t_{n}+\sigma_{n} T t_{n}-p\right\| \\
& =\left\|\beta_{n}\left(x_{n}-p\right)+\gamma_{n}\left(t_{n}-p\right)+\sigma_{n}\left(T t_{n}-p\right)\right\| \\
& \leq \beta_{n}\left\|x_{n}-p\right\|+\left\|\gamma_{n}\left(t_{n}-p\right)+\sigma_{n}\left(T t_{n}-p\right)\right\| \\
& \leq \beta_{n}\left\|x_{n}-p\right\|+\left(\gamma_{n}+\sigma_{n}\right)\left\|t_{n}-p\right\| \\
& \leq \beta_{n}\left\|x_{n}-p\right\|+\left(\gamma_{n}+\sigma_{n}\right)\left[\left\|x_{n}-p\right\|+\lambda_{n} \alpha_{n}\|p\|\right] \\
& \leq \beta_{n}\left\|x_{n}-p\right\|+\left(\gamma_{n}+\delta_{n}\right)\left\|x_{n}-p\right\|+\lambda_{n} \alpha_{n}\|p\| \\
& =\left\|x_{n}-p\right\|+\lambda_{n} \alpha_{n}\|p\| .
\end{aligned}
$$

Noticing the boundedness of $\left\{S x_{n}\right\}$, we get $\sup _{n \geq 0}\left\|\gamma S x_{n}-\mu F p\right\| \leq \widehat{M}$ for some $\widehat{M}>0$. Moreover, utilizing Lemma 2.4 and (3.1), (3.6), we deduce that $\left\{\lambda_{n}\right\} \subset[a, b] \subset\left(0, \frac{2}{\|A\|^{2}}\right)$ and $0<\gamma \leq \tau$ that, for all $n \geq 0$,

$$
\begin{aligned}
& \left\|x_{n+1}-p\right\| \\
& =\left\|\epsilon_{n} \gamma\left(\delta_{n} V x_{n}+\left(1-\delta_{n}\right) S x_{n}\right)+\left(I-\epsilon_{n} \mu F\right) y_{n}-p\right\| \\
& =\left\|\epsilon_{n} \gamma\left(\delta_{n} V x_{n}+\left(1-\delta_{n}\right) S x_{n}\right)-\epsilon_{n} \mu F p+\left(I-\epsilon_{n} \mu F\right) y_{n}-\left(I-\epsilon_{n} \mu F\right) p\right\| \\
& \leq\left\|\epsilon_{n} \gamma\left(\delta_{n} V x_{n}+\left(1-\delta_{n}\right) S x_{n}\right)-\epsilon_{n} \mu F p\right\|+\left\|\left(I-\epsilon_{n} \mu F\right) y_{n}-\left(I-\epsilon_{n} \mu F\right) p\right\| \\
& =\epsilon_{n}\left\|\delta_{n}\left(\gamma V x_{n}-\mu F p\right)+\left(1-\delta_{n}\right)\left(\gamma S x_{n}-\mu F p\right)\right\| \\
& +\left\|\left(I-\epsilon_{n} \mu F\right) y_{n}-\left(I-\epsilon_{n} \mu F\right) p\right\| \\
& \leq \epsilon_{n}\left[\delta_{n}\left\|\gamma V x_{n}-\mu F p\right\|+\left(1-\delta_{n}\right)\left\|\gamma S x_{n}-\mu F p\right\|\right]+\left(1-\epsilon_{n} \tau\right)\left\|y_{n}-p\right\| \\
& \leq \epsilon_{n}\left[\delta_{n}\left(\left\|\gamma V x_{n}-\gamma V p\right\|+\|\gamma V p-\mu F p\|\right)+\left(1-\delta_{n}\right) \widehat{M}\right]+\left(1-\epsilon_{n} \tau\right)\left\|y_{n}-p\right\| \\
& \leq \epsilon_{n}\left[\delta_{n} \gamma \rho\left\|x_{n}-p\right\|+\delta_{n}\|\gamma V p-\mu F p\|+\left(1-\delta_{n}\right) \widehat{M}\right] \\
& +\left(1-\epsilon_{n} \tau\right)\left[\left\|x_{n}-p\right\|+\lambda_{n} \alpha_{n}\|p\|\right] \\
& \leq \epsilon_{n}\left[\delta_{n} \gamma \rho\left\|x_{n}-p\right\|+\max \{\widehat{M},\|\gamma V p-\mu F p\|\}\right]+\left(1-\epsilon_{n} \tau\right)\left[\left\|x_{n}-p\right\|+\lambda_{n} \alpha_{n}\|p\|\right] \\
& \leq \epsilon_{n} \gamma \rho\left\|x_{n}-p\right\|+\epsilon_{n} \max \{\widehat{M},\|\gamma V p-\mu F p\|\}+\left(1-\epsilon_{n} \tau\right)\left\|x_{n}-p\right\|+\lambda_{n} \alpha_{n}\|p\| \\
& =\left[1-(\tau-\gamma \rho) \epsilon_{n}\right]\left\|x_{n}-p\right\|+\epsilon_{n} \max \{\widehat{M},\|\gamma V p-\mu F p\|\}+\lambda_{n} \alpha_{n}\|p\| \\
& =\left[1-(\tau-\gamma \rho) \epsilon_{n}\right]\left\|x_{n}-p\right\|+(\tau-\gamma \rho) \epsilon_{n} \max \left\{\frac{\widehat{M}}{\tau-\gamma \rho}, \frac{\|\gamma V p-\mu F p\|}{\tau-\gamma \rho}\right\}+\lambda_{n} \alpha_{n}\|p\| \\
& \leq \max \left\{\left\|x_{n}-p\right\|, \frac{\widehat{M}}{\tau-\gamma \rho}, \frac{\|\gamma V p-\mu F p\|}{\tau-\gamma \rho}\right\}+\alpha_{n} b\|p\| \text {. }
\end{aligned}
$$

By induction, we get

$$
\left\|x_{n+1}-p\right\| \leq \max \left\{\left\|x_{0}-p\right\|, \frac{\widehat{M}}{\tau-\gamma \rho}, \frac{\|\gamma V p-\mu F p\|}{\tau-\gamma \rho}\right\}+\sum_{j=0}^{n} \alpha_{j} b\|p\|, \quad \forall n \geq 0
$$


Thus, $\left\{x_{n}\right\}$ is bounded since $\sum_{n=0}^{\infty} \alpha_{n}<\infty$, and so are the sequences $\left\{t_{n}\right\},\left\{u_{n}\right\}$, $\left\{v_{n}\right\}$, and $\left\{y_{n}\right\}$.

Step 2. We prove that $\lim _{n \rightarrow \infty} \frac{\left\|x_{n+1}-x_{n}\right\|}{\epsilon_{n}}=0$.

Indeed, utilizing (2.1) and (2.3), we obtain

$$
\begin{aligned}
& \left\|v_{n+1}-v_{n}\right\| \\
& =\left\|\Lambda_{n+1}^{N} u_{n+1}-\Lambda_{n}^{N} u_{n}\right\| \\
& =\left\|J_{R_{N}, \lambda_{N, n+1}}\left(I-\lambda_{N, n+1} B_{N}\right) \Lambda_{n+1}^{N-1} u_{n+1}-J_{R_{N}, \lambda_{N, n}}\left(I-\lambda_{N, n} B_{N}\right) \Lambda_{n}^{N-1} u_{n}\right\| \\
& \leq\left\|J_{R_{N}, \lambda_{N, n+1}}\left(I-\lambda_{N, n+1} B_{N}\right) \Lambda_{n+1}^{N-1} u_{n+1}-J_{R_{N}, \lambda_{N, n+1}}\left(I-\lambda_{N, n} B_{N}\right) \Lambda_{n+1}^{N-1} u_{n+1}\right\| \\
& +\left\|J_{R_{N}, \lambda_{N, n+1}}\left(I-\lambda_{N, n} B_{N}\right) \Lambda_{n+1}^{N-1} u_{n+1}-J_{R_{N}, \lambda_{N, n}}\left(I-\lambda_{N, n} B_{N}\right) \Lambda_{n}^{N-1} u_{n}\right\| \\
& \leq\left\|\left(I-\lambda_{N, n+1} B_{N}\right) \Lambda_{n+1}^{N-1} u_{n+1}-\left(I-\lambda_{N, n} B_{N}\right) \Lambda_{n+1}^{N-1} u_{n+1}\right\| \\
& +\left\|\left(I-\lambda_{N, n} B_{N}\right) \Lambda_{n+1}^{N-1} u_{n+1}-\left(I-\lambda_{N, n} B_{N}\right) \Lambda_{n}^{N-1} u_{n}\right\|+\left|\lambda_{N, n+1}-\lambda_{N, n}\right| \\
& \times\left(\frac{1}{\lambda_{N, n+1}}\left\|J_{R_{N}, \lambda_{N, n+1}}\left(I-\lambda_{N, n} B_{N}\right) \Lambda_{n+1}^{N-1} u_{n+1}-\left(I-\lambda_{N, n} B_{N}\right) \Lambda_{n}^{N-1} u_{n}\right\|\right. \\
& \left.+\frac{1}{\lambda_{N, n}}\left\|\left(I-\lambda_{N, n} B_{N}\right) \Lambda_{n+1}^{N-1} u_{n+1}-J_{R_{N}, \lambda_{N, n}}\left(I-\lambda_{N, n} B_{N}\right) \Lambda_{n}^{N-1} u_{n}\right\|\right) \\
& \leq\left|\lambda_{N, n+1}-\lambda_{N, n}\right|\left(\left\|B_{N} \Lambda_{n+1}^{N-1} u_{n+1}\right\|+\widetilde{M}\right)+\left\|\Lambda_{n+1}^{N-1} u_{n+1}-\Lambda_{n}^{N-1} u_{n}\right\| \\
& \leq\left|\lambda_{N, n+1}-\lambda_{N, n}\right|\left(\left\|B_{N} \Lambda_{n+1}^{N-1} u_{n+1}\right\|+\tilde{M}\right) \\
& +\left|\lambda_{N-1, n+1}-\lambda_{N-1, n}\right|\left(\left\|B_{N-1} \Lambda_{n+1}^{N-2} u_{n+1}\right\|+\tilde{M}\right)+\left\|\Lambda_{n+1}^{N-2} u_{n+1}-\Lambda_{n}^{N-2} u_{n}\right\| \\
& \vdots \\
& \leq\left|\lambda_{N, n+1}-\lambda_{N, n}\right|\left(\left\|B_{N} \Lambda_{n+1}^{N-1} u_{n+1}\right\|+\tilde{M}\right) \\
& +\left|\lambda_{N-1, n+1}-\lambda_{N-1, n}\right|\left(\left\|B_{N-1} \Lambda_{n+1}^{N-2} u_{n+1}\right\|+\tilde{M}\right) \\
& +\cdots+\left|\lambda_{1, n+1}-\lambda_{1, n}\right|\left(\left\|B_{1} \Lambda_{n+1}^{0} u_{n+1}\right\|+\tilde{M}\right)+\left\|\Lambda_{n+1}^{0} u_{n+1}-\Lambda_{n}^{0} u_{n}\right\| \\
& \leq \tilde{M}_{0} \sum_{i=1}^{N}\left|\lambda_{i, n+1}-\lambda_{i, n}\right|+\left\|u_{n+1}-u_{n}\right\|,
\end{aligned}
$$

where

$$
\begin{aligned}
& \sup _{n \geq 0}\left\{\frac{1}{\lambda_{N, n+1}}\left\|J_{R_{N}, \lambda_{N, n+1}}\left(I-\lambda_{N, n} B_{N}\right) \Lambda_{n+1}^{N-1} u_{n+1}-\left(I-\lambda_{N, n} B_{N}\right) \Lambda_{n}^{N-1} u_{n}\right\|\right. \\
& \left.\quad+\frac{1}{\lambda_{N, n}}\left\|\left(I-\lambda_{N, n} B_{N}\right) \Lambda_{n+1}^{N-1} u_{n+1}-J_{R_{N}, \lambda_{N, n}}\left(I-\lambda_{N, n} B_{N}\right) \Lambda_{n}^{N-1} u_{n}\right\|\right\} \leq \tilde{M}
\end{aligned}
$$

for some $\tilde{M}>0$ and $\sup _{n \geq 0}\left\{\sum_{i=1}^{N}\left\|B_{i} \Lambda_{n+1}^{i-1} u_{n+1}\right\|+\tilde{M}\right\} \leq \widetilde{M}_{0}$ for some $\widetilde{M}_{0}>0$.

Utilizing Proposition 2.4(b), (e), we deduce that

$$
\begin{aligned}
& \left\|u_{n+1}-u_{n}\right\| \\
& =\left\|\Delta_{n+1}^{M} x_{n+1}-\Delta_{n}^{M} x_{n}\right\|
\end{aligned}
$$

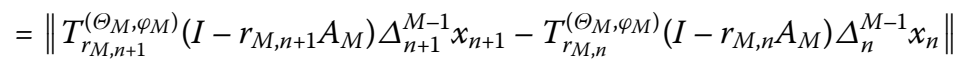




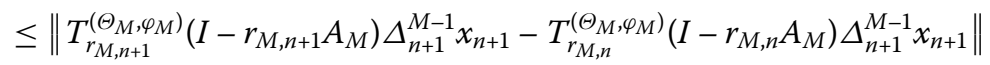

$$
\begin{aligned}
& +\left\|T_{r_{M, n}}^{\left(\Theta_{M}, \varphi_{M}\right)}\left(I-r_{M, n} A_{M}\right) \Delta_{n+1}^{M-1} x_{n+1}-T_{r_{M, n}}^{\left(\Theta_{M}, \varphi_{M}\right)}\left(I-r_{M, n} A_{M}\right) \Delta_{n}^{M-1} x_{n}\right\|
\end{aligned}
$$

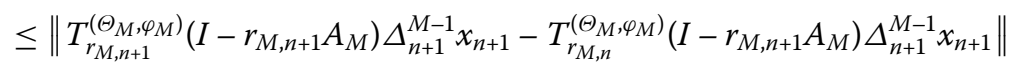

$$
\begin{aligned}
& +\left\|T_{r_{M, n}}^{\left(\Theta_{M}, \varphi_{M}\right)}\left(I-r_{M, n+1} A_{M}\right) \Delta_{n+1}^{M-1} x_{n+1}-T_{r_{M, n}}^{\left(\Theta_{M}, \varphi_{M}\right)}\left(I-r_{M, n} A_{M}\right) \Delta_{n+1}^{M-1} x_{n+1}\right\| \\
& +\left\|\left(I-r_{M, n} A_{M}\right) \Delta_{n+1}^{M-1} x_{n+1}-\left(I-r_{M, n} A_{M}\right) \Delta_{n}^{M-1} x_{n}\right\| \\
& \leq \frac{\left|r_{M, n+1}-r_{M, n}\right|}{r_{M, n+1}}\left\|T_{r_{M, n+1}}^{\left(\Theta_{M}, \varphi_{M}\right)}\left(I-r_{M, n+1} A_{M}\right) \Delta_{n+1}^{M-1} x_{n+1}-\left(I-r_{M, n+1} A_{M}\right) \Delta_{n+1}^{M-1} x_{n+1}\right\| \\
& +\left|r_{M, n+1}-r_{M, n}\right|\left\|A_{M} \Delta_{n+1}^{M-1} x_{n+1}\right\|+\left\|\Delta_{n+1}^{M-1} x_{n+1}-\Delta_{n}^{M-1} x_{n}\right\|
\end{aligned}
$$

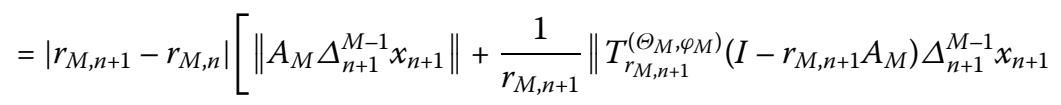

$$
\begin{aligned}
& \left.-\left(I-r_{M, n+1} A_{M}\right) \Delta_{n+1}^{M-1} x_{n+1} \|\right]+\left\|\Delta_{n+1}^{M-1} x_{n+1}-\Delta_{n}^{M-1} x_{n}\right\| \\
& \vdots
\end{aligned}
$$

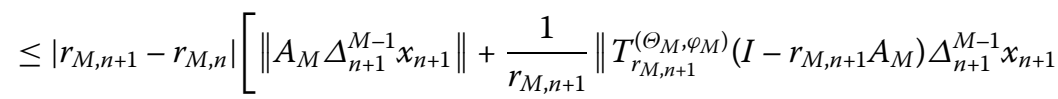

$$
\begin{aligned}
& \left.-\left(I-r_{M, n+1} A_{M}\right) \Delta_{n+1}^{M-1} x_{n+1} \|\right]+\cdots+\left|r_{1, n+1}-r_{1, n}\right|\left[\left\|A_{1} \Delta_{n+1}^{0} x_{n+1}\right\|\right. \\
& \left.+\frac{1}{r_{1, n+1}}\left\|T_{r_{1, n+1}}^{\left(\Theta_{1}, \varphi_{1}\right)}\left(I-r_{1, n+1} A_{1}\right) \Delta_{n+1}^{0} x_{n+1}-\left(I-r_{1, n+1} A_{1}\right) \Delta_{n+1}^{0} x_{n+1}\right\|\right] \\
& +\left\|\Delta_{n+1}^{0} x_{n+1}-\Delta_{n}^{0} x_{n}\right\| \\
& \leq \widetilde{M}_{1} \sum_{k=1}^{M}\left|r_{k, n+1}-r_{k, n}\right|+\left\|x_{n+1}-x_{n}\right\|
\end{aligned}
$$

where $\widetilde{M}_{1}>0$ is a constant such that, for each $n \geq 0$,

$$
\begin{aligned}
& \sum_{k=1}^{M}\left[\left\|A_{k} \Delta_{n+1}^{k-1} x_{n+1}\right\|+\frac{1}{r_{k, n+1}}\left\|T_{r_{k, n+1}}^{\left(\Theta_{k}, \varphi_{k}\right)}\left(I-r_{k, n+1} A_{k}\right) \Delta_{n+1}^{k-1} x_{n+1}-\left(I-r_{k, n+1} A_{k}\right) \Delta_{n+1}^{k-1} x_{n+1}\right\|\right] \\
& \quad \leq \tilde{M}_{1} .
\end{aligned}
$$

Furthermore, we define $y_{n}=\beta_{n} x_{n}+\left(1-\beta_{n}\right) w_{n}$ for all $n \geq 0$. It follows that

$$
\begin{aligned}
& w_{n+1}-w_{n} \\
& =\frac{y_{n+1}-\beta_{n+1} x_{n+1}}{1-\beta_{n+1}}-\frac{y_{n}-\beta_{n} x_{n}}{1-\beta_{n}} \\
& =\frac{\gamma_{n+1} t_{n+1}+\sigma_{n+1} T t_{n+1}}{1-\beta_{n+1}}-\frac{\gamma_{n} t_{n}+\sigma_{n} T t_{n}}{1-\beta_{n}} \\
& =\frac{\gamma_{n+1}\left(t_{n+1}-t_{n}\right)+\sigma_{n+1}\left(T t_{n+1}-T t_{n}\right)}{1-\beta_{n+1}}+\left(\frac{\gamma_{n+1}}{1-\beta_{n+1}}-\frac{\gamma_{n}}{1-\beta_{n}}\right) t_{n} \\
& \quad+\left(\frac{\sigma_{n+1}}{1-\beta_{n+1}}-\frac{\sigma_{n}}{1-\beta_{n}}\right) T t_{n} .
\end{aligned}
$$


Since $T$ is a $\xi$-strictly pseudocontractive mapping and $\left(\gamma_{n}+\sigma_{n}\right) \xi \leq \gamma_{n}$, for all $n \geq 0$, by Lemma 2.2, we obtain

$$
\left\|\gamma_{n+1}\left(t_{n+1}-t_{n}\right)+\sigma_{n+1}\left(T t_{n+1}-T t_{n}\right)\right\| \leq\left(\gamma_{n+1}+\sigma_{n+1}\right)\left\|t_{n+1}-t_{n}\right\|
$$

Also, utilizing the nonexpansivity of $P_{C}\left(I-\lambda_{n} \nabla f_{\alpha_{n}}\right)$, we have

$$
\begin{aligned}
\left\|t_{n+1}-t_{n}\right\|= & \left\|P_{C}\left(I-\lambda_{n+1} \nabla f_{\alpha_{n+1}}\right) v_{n+1}-P_{C}\left(I-\lambda_{n} \nabla f_{\alpha_{n}}\right) v_{n}\right\| \\
\leq & \left\|P_{C}\left(I-\lambda_{n+1} \nabla f_{\alpha_{n+1}}\right) v_{n+1}-P_{C}\left(I-\lambda_{n+1} \nabla f_{\alpha_{n+1}}\right) v_{n}\right\| \\
& \quad+\left\|P_{C}\left(I-\lambda_{n+1} \nabla f_{\alpha_{n+1}}\right) v_{n}-P_{C}\left(I-\lambda_{n} \nabla f_{\alpha_{n}}\right) v_{n}\right\| \\
\leq & \left\|v_{n+1}-v_{n}\right\|+\left\|\left(I-\lambda_{n+1} \nabla f_{\alpha_{n+1}}\right) v_{n}-\left(I-\lambda_{n} \nabla f_{\alpha_{n}}\right) v_{n}\right\| \\
\leq & \left\|v_{n+1}-v_{n}\right\|+\left|\lambda_{n+1} \alpha_{n+1}-\lambda_{n} \alpha_{n}\right|\left\|v_{n}\right\| \\
& +\left|\lambda_{n+1}-\lambda_{n}\right|\left\|\nabla f\left(v_{n}\right)\right\| .
\end{aligned}
$$

Hence, from (3.7)-(3.11), it follows that

$$
\begin{aligned}
& \left\|w_{n+1}-w_{n}\right\| \\
& \leq \frac{\left\|\gamma_{n+1}\left(t_{n+1}-t_{n}\right)+\sigma_{n+1}\left(T t_{n+1}-T t_{n}\right)\right\|}{1-\beta_{n+1}} \\
& +\left|\frac{\gamma_{n+1}}{1-\beta_{n+1}}-\frac{\gamma_{n}}{1-\beta_{n}}\right|\left\|t_{n}\right\|+\left|\frac{\sigma_{n+1}}{1-\beta_{n+1}}-\frac{\sigma_{n}}{1-\beta_{n}}\right|\left\|T t_{n}\right\| \\
& \leq \frac{\left(\gamma_{n+1}+\sigma_{n+1}\right)\left\|t_{n+1}-t_{n}\right\|}{1-\beta_{n+1}}+\left|\frac{\gamma_{n+1}}{1-\beta_{n+1}}-\frac{\gamma_{n}}{1-\beta_{n}}\right|\left\|t_{n}\right\|+\left|\frac{\sigma_{n+1}}{1-\beta_{n+1}}-\frac{\sigma_{n}}{1-\beta_{n}}\right|\left\|T t_{n}\right\| \\
& =\left\|t_{n+1}-t_{n}\right\|+\left|\frac{\gamma_{n+1}}{1-\beta_{n+1}}-\frac{\gamma_{n}}{1-\beta_{n}}\right|\left(\left\|t_{n}\right\|+\left\|T t_{n}\right\|\right) \\
& \leq\left\|v_{n+1}-v_{n}\right\|+\left|\lambda_{n+1} \alpha_{n+1}-\lambda_{n} \alpha_{n}\right|\left\|v_{n}\right\|+\left|\lambda_{n+1}-\lambda_{n}\right|\left\|\nabla f\left(v_{n}\right)\right\| \\
& +\left|\frac{\gamma_{n+1}}{1-\beta_{n+1}}-\frac{\gamma_{n}}{1-\beta_{n}}\right|\left(\left\|t_{n}\right\|+\left\|T t_{n}\right\|\right) \\
& \leq \widetilde{M}_{0} \sum_{i=1}^{N}\left|\lambda_{i, n+1}-\lambda_{i, n}\right|+\left\|u_{n+1}-u_{n}\right\|+\left|\lambda_{n+1} \alpha_{n+1}-\lambda_{n} \alpha_{n}\right|\left\|v_{n}\right\| \\
& +\left|\lambda_{n+1}-\lambda_{n}\right|\left\|\nabla f\left(v_{n}\right)\right\|+\left|\frac{\gamma_{n+1}}{1-\beta_{n+1}}-\frac{\gamma_{n}}{1-\beta_{n}}\right|\left(\left\|t_{n}\right\|+\left\|T t_{n}\right\|\right) \\
& \leq \tilde{M}_{0} \sum_{i=1}^{N}\left|\lambda_{i, n+1}-\lambda_{i, n}\right|+\tilde{M}_{1} \sum_{k=1}^{M}\left|r_{k, n+1}-r_{k, n}\right|+\left\|x_{n+1}-x_{n}\right\| \\
& +\left|\lambda_{n+1} \alpha_{n+1}-\lambda_{n} \alpha_{n}\right|\left\|v_{n}\right\|+\left|\lambda_{n+1}-\lambda_{n}\right|\left\|\nabla f\left(v_{n}\right)\right\| \\
& +\left|\frac{\gamma_{n+1}}{1-\beta_{n+1}}-\frac{\gamma_{n}}{1-\beta_{n}}\right|\left(\left\|t_{n}\right\|+\left\|T t_{n}\right\|\right) \text {. }
\end{aligned}
$$

In the meantime, simple calculation shows that

$$
y_{n+1}-y_{n}=\beta_{n}\left(x_{n+1}-x_{n}\right)+\left(1-\beta_{n}\right)\left(w_{n+1}-w_{n}\right)+\left(\beta_{n+1}-\beta_{n}\right)\left(x_{n+1}-w_{n+1}\right) .
$$


So, it follows from (3.12) that

$$
\begin{aligned}
\left\|y_{n+1}-y_{n}\right\| & \\
\leq & \beta_{n}\left\|x_{n+1}-x_{n}\right\|+\left(1-\beta_{n}\right)\left\|w_{n+1}-w_{n}\right\|+\left|\beta_{n+1}-\beta_{n}\right|\left\|x_{n+1}-w_{n+1}\right\| \\
\leq & \beta_{n}\left\|x_{n+1}-x_{n}\right\|+\left(1-\beta_{n}\right)\left[\tilde{M}_{0} \sum_{i=1}^{N}\left|\lambda_{i, n+1}-\lambda_{i, n}\right|+\tilde{M}_{1} \sum_{k=1}^{M}\left|r_{k, n+1}-r_{k, n}\right|\right. \\
& +\left\|x_{n+1}-x_{n}\right\|+\left|\frac{\gamma_{n+1}}{1-\beta_{n+1}}-\frac{\gamma_{n}}{1-\beta_{n}}\right|\left(\left\|t_{n}\right\|+\left\|T t_{n}\right\|\right)+\left|\lambda_{n+1} \alpha_{n+1}-\lambda_{n} \alpha_{n}\right|\left\|v_{n}\right\| \\
& \left.+\left|\lambda_{n+1}-\lambda_{n}\right|\left\|\nabla f\left(v_{n}\right)\right\|\right]+\left|\beta_{n+1}-\beta_{n}\right|\left\|x_{n+1}-w_{n+1}\right\| \\
\leq & \left\|x_{n+1}-x_{n}\right\|+\tilde{M}_{0} \sum_{i=1}^{N}\left|\lambda_{i, n+1}-\lambda_{i, n}\right|+\tilde{M}_{1} \sum_{k=1}^{M}\left|r_{k, n+1}-r_{k, n}\right| \\
& +\frac{\left|\gamma_{n+1}-\gamma_{n}\right|\left(1-\beta_{n}\right)+\gamma_{n}\left|\beta_{n+1}-\beta_{n}\right|}{1-\beta_{n+1}}\left(\left\|t_{n}\right\|+\left\|T t_{n}\right\|\right) \\
& +\left|\lambda_{n+1} \alpha_{n+1}-\lambda_{n} \alpha_{n}\right|\left|v_{n}\left\|+\left|\lambda_{n+1}-\lambda_{n}\right|\right\| \nabla f\left(v_{n}\right)\left\|+\left|\beta_{n+1}-\beta_{n}\right|\right\| x_{n+1}-w_{n+1} \|\right. \\
\leq & \left\|x_{n+1}-x_{n}\right\|+\tilde{M}_{0} \sum_{i=1}^{N}\left|\lambda_{i, n+1}-\lambda_{i, n}\right|+\tilde{M}_{1} \sum_{k=1}^{M}\left|r_{k, n+1}-r_{k, n}\right| \\
& +\left|\gamma_{n+1}-\gamma_{n}\right| \frac{\left\|t_{n}\right\|+\left\|T t_{n}\right\|}{1-d}+\left|\beta_{n+1}-\beta_{n}\right|\left(\left\|x_{n+1}-w_{n+1}\right\|+\frac{\left\|t_{n}\right\|+\left\|T t_{n}\right\|}{1-d}\right) \\
& +\left|\lambda_{n+1} \alpha_{n+1}-\lambda_{n} \alpha_{n}\right|\left\|v_{n}\right\|+\left|\lambda_{n+1}-\lambda_{n}\right|\left\|\nabla f\left(v_{n}\right)\right\| \\
\leq & \left\|x_{n+1}-x_{n}\right\|+\tilde{M}_{2}\left(\sum_{i=1}^{N}\left|\lambda_{i, n+1}-\lambda_{i, n}\right|+\sum_{k=1}^{M}\left|r_{k, n+1}-r_{k, n}\right|\right. \\
& \left.+\gamma_{n}|+| \beta_{n+1}-\beta_{n}|+| \lambda_{n+1} \alpha_{n+1}-\lambda_{n} \alpha_{n}|+| \lambda_{n+1}-\lambda_{n} \mid\right),
\end{aligned}
$$

where $\sup _{n \geq 0}\left\{\left\|x_{n+1}-w_{n+1}\right\|+\frac{\left\|t_{n}\right\|+\left\|T t_{n}\right\|}{1-d}+\left\|v_{n}\right\|+\left\|\nabla f\left(v_{n}\right)\right\|+\widetilde{M}_{0}+\widetilde{M}_{1}\right\} \leq \widetilde{M}_{2}$ for some $\widetilde{M}_{2}>0$.

On the other hand, we define $z_{n}:=\delta_{n} V x_{n}+\left(1-\delta_{n}\right) S x_{n}$, for all $n \geq 0$. Then it is well known that $x_{n+1}=\epsilon_{n} \gamma z_{n}+\left(I-\epsilon_{n} \mu F\right) y_{n}$, for all $n \geq 0$. Simple calculations show that

$$
\left\{\begin{aligned}
z_{n+1}-z_{n}= & \left(\delta_{n+1}-\delta_{n}\right)\left(V x_{n}-S x_{n}\right)+\delta_{n+1}\left(V x_{n+1}-V x_{n}\right) \\
+ & \left(1-\delta_{n+1}\right)\left(S x_{n+1}-S x_{n}\right), \\
x_{n+2}-x_{n+1}= & \left(\epsilon_{n+1}-\epsilon_{n}\right)\left(\gamma z_{n}-\mu F y_{n}\right)+\epsilon_{n+1} \gamma\left(z_{n+1}-z_{n}\right) \\
& +\left(I-\lambda_{n+1} \mu F\right) y_{n+1}-\left(I-\lambda_{n+1} \mu F\right) y_{n} .
\end{aligned}\right.
$$

Since $V$ is a $\rho$-contraction with coefficient $\rho \in[0,1)$ and $S$ is a nonexpansive mapping, we conclude that

$$
\begin{aligned}
\left\|z_{n+1}-z_{n}\right\| \leq & \left|\delta_{n+1}-\delta_{n}\right|\left\|V x_{n}-S x_{n}\right\|+\delta_{n+1}\left\|V x_{n+1}-V x_{n}\right\| \\
& +\left(1-\delta_{n+1}\right)\left\|S x_{n+1}-S x_{n}\right\| \\
\leq & \left|\delta_{n+1}-\delta_{n}\right|\left\|V x_{n}-S x_{n}\right\|+\delta_{n+1} \rho\left\|x_{n+1}-x_{n}\right\|
\end{aligned}
$$




$$
\begin{aligned}
& +\left(1-\delta_{n+1}\right)\left\|x_{n+1}-x_{n}\right\| \\
= & \left(1-\delta_{n+1}(1-\rho)\right)\left\|x_{n+1}-x_{n}\right\|+\left|\delta_{n+1}-\delta_{n}\right|\left\|V x_{n}-S x_{n}\right\|,
\end{aligned}
$$

which together with (3.13) and $0<\gamma \leq \tau$ implies that

$$
\begin{aligned}
\| x_{n+2}- & x_{n+1} \| \\
\leq & \left|\epsilon_{n+1}-\epsilon_{n}\right|\left\|\gamma z_{n}-\mu F y_{n}\right\|+\epsilon_{n+1} \gamma\left\|z_{n+1}-z_{n}\right\| \\
& +\left\|\left(I-\epsilon_{n+1} \mu F\right) y_{n+1}-\left(I-\epsilon_{n+1} \mu F\right) y_{n}\right\| \\
\leq & \left|\epsilon_{n+1}-\epsilon_{n}\right|\left\|\gamma z_{n}-\mu F y_{n}\right\|+\epsilon_{n+1} \gamma\left\|z_{n+1}-z_{n}\right\|+\left(1-\epsilon_{n+1} \tau\right)\left\|y_{n+1}-y_{n}\right\| \\
\leq & \left|\epsilon_{n+1}-\epsilon_{n}\right|\left\|\gamma z_{n}-\mu F y_{n}\right\|+\epsilon_{n+1} \gamma\left[\left(1-\delta_{n+1}(1-\rho)\right)\left\|x_{n+1}-x_{n}\right\|\right. \\
& \left.+\left|\delta_{n+1}-\delta_{n}\right||| V x_{n}-S x_{n} \|\right]+\left(1-\epsilon_{n+1} \tau\right)\left[\left\|x_{n+1}-x_{n}\right\|\right. \\
& +\widetilde{M}_{2}\left(\sum_{i=1}^{N}\left|\lambda_{i, n+1}-\lambda_{i, n}\right|+\sum_{k=1}^{M}\left|r_{k, n+1}-r_{k, n}\right|+\left|\gamma_{n+1}-\gamma_{n}\right|+\left|\beta_{n+1}-\beta_{n}\right|\right. \\
& \left.\left.+\left|\lambda_{n+1} \alpha_{n+1}-\lambda_{n} \alpha_{n}\right|+\left|\lambda_{n+1}-\lambda_{n}\right|\right)\right] \\
\leq & \left(1-\epsilon_{n+1}(\tau-\gamma)-\epsilon_{n+1} \delta_{n+1}(1-\rho) \gamma\right)\left\|x_{n+1}-x_{n}\right\|+\left|\epsilon_{n+1}-\epsilon_{n}\right|\left\|\gamma z_{n}-\mu F y_{n}\right\| \\
& +\epsilon_{n+1}\left|\delta_{n+1}-\delta_{n}\right|\left\|V x_{n}-S x_{n}\right\|+\widetilde{M}_{2}\left(\sum_{i=1}^{N}\left|\lambda_{i, n+1}-\lambda_{i, n}\right|+\sum_{k=1}^{M}\left|r_{k, n+1}-r_{k, n}\right|\right. \\
& \left.+\left|\gamma_{n+1}-\gamma_{n}\right|+\left|\beta_{n+1}-\beta_{n}\right|+\left|\lambda_{n+1} \alpha_{n+1}-\lambda_{n} \alpha_{n}\right|+\left|\lambda_{n+1}-\lambda_{n}\right|\right) \\
& +\left(1-\epsilon_{n+1} \delta_{n+1}(1-\rho) \gamma\right)\left\|x_{n+1}-x_{n}\right\|+\widetilde{M}_{3}\left\{\sum_{i=1}^{N}\left|\lambda_{i, n+1}-\lambda_{i, n}\right|\right. \\
& +\sum_{k=1}^{M}\left|r_{k, n+1}-r_{k, n}\right|+\left|\epsilon_{n+1}-\epsilon_{n}\right|+\epsilon_{n+1}\left|\delta_{n+1}-\delta_{n}\right|+\left|\beta_{n+1}-\beta_{n}\right| \\
& \left.+\left|\lambda_{n+1} \alpha_{n+1}-\lambda_{n} \alpha_{n}\right|+\left|\lambda_{n+1}-\lambda_{n}\right|\right\}
\end{aligned}
$$

where $\sup _{n \geq 0}\left\{\left\|\gamma z_{n}-\mu F y_{n}\right\|+\left\|V x_{n}-S x_{n}\right\|+\widetilde{M}_{2}\right\} \leq \widetilde{M}_{3}$ for some $\widetilde{M}_{3}>0$. Consequently,

$$
\begin{aligned}
& \frac{\left\|x_{n+1}-x_{n}\right\|}{\epsilon_{n}} \\
& \leq\left(1-\epsilon_{n} \delta_{n}(1-\rho) \gamma\right) \frac{\left\|x_{n}-x_{n-1}\right\|}{\epsilon_{n}}+\widetilde{M}_{3}\left\{\sum_{i=1}^{N} \frac{\left|\lambda_{i, n}-\lambda_{i, n-1}\right|}{\epsilon_{n}}+\sum_{k=1}^{M} \frac{\left|r_{k, n}-r_{k, n-1}\right|}{\epsilon_{n}}\right. \\
& \quad+\frac{\left|\epsilon_{n}-\epsilon_{n-1}\right|}{\epsilon_{n}}+\left|\delta_{n}-\delta_{n-1}\right|+\frac{\left|\beta_{n}-\beta_{n-1}\right|}{\epsilon_{n}}+\frac{\left|\gamma_{n}-\gamma_{n-1}\right|}{\epsilon_{n}} \\
& \left.\quad+\frac{\left|\lambda_{n} \alpha_{n}-\lambda_{n-1} \alpha_{n-1}\right|}{\epsilon_{n}}+\frac{\left|\lambda_{n}-\lambda_{n-1}\right|}{\epsilon_{n}}\right\}
\end{aligned}
$$




$$
\begin{aligned}
= & \left(1-\epsilon_{n} \delta_{n}(1-\rho) \gamma\right) \frac{|| x_{n}-x_{n-1} \|}{\epsilon_{n-1}}+\left(1-\epsilon_{n} \delta_{n}(1-\rho) \gamma\right)\left\|x_{n}-x_{n-1}\right\|\left(\frac{1}{\epsilon_{n}}-\frac{1}{\epsilon_{n-1}}\right) \\
& +\widetilde{M}_{3}\left\{\sum_{i=1}^{N} \frac{\left|\lambda_{i, n}-\lambda_{i, n-1}\right|}{\epsilon_{n}}+\sum_{k=1}^{M} \frac{\left|r_{k, n}-r_{k, n-1}\right|}{\epsilon_{n}}+\frac{\left|\epsilon_{n}-\epsilon_{n-1}\right|}{\epsilon_{n}}+\left|\delta_{n}-\delta_{n-1}\right|\right. \\
& \left.+\frac{\left|\beta_{n}-\beta_{n-1}\right|}{\epsilon_{n}}+\frac{\left|\gamma_{n}-\gamma_{n-1}\right|}{\epsilon_{n}}+\frac{\left|\lambda_{n} \alpha_{n}-\lambda_{n-1} \alpha_{n-1}\right|}{\epsilon_{n}}+\frac{\left|\lambda_{n}-\lambda_{n-1}\right|}{\epsilon_{n}}\right\} \\
\leq & \left(1-\epsilon_{n} \delta_{n}(1-\rho) \gamma\right) \frac{|| x_{n}-x_{n-1} \|}{\epsilon_{n-1}}+\epsilon_{n} \delta_{n}(1-\rho) \gamma \cdot \frac{\tilde{M}_{4}}{(1-\rho) \gamma}\left\{\frac{\left|\epsilon_{n}-\epsilon_{n-1}\right|}{\delta_{n} \epsilon_{n}^{2} \epsilon_{n-1}}\right. \\
& +\sum_{i=1}^{N} \frac{\left|\lambda_{i, n}-\lambda_{i, n-1}\right|}{\delta_{n} \epsilon_{n}^{2}}+\sum_{k=1}^{M} \frac{\left|r_{k, n}-r_{k, n-1}\right|}{\delta_{n} \epsilon_{n}^{2}}+\frac{\left|\epsilon_{n}-\epsilon_{n-1}\right|}{\delta_{n} \epsilon_{n}^{2}}+\frac{\left|\delta_{n}-\delta_{n-1}\right|}{\delta_{n} \epsilon_{n}} \\
& \left.+\frac{\left|\beta_{n}-\beta_{n-1}\right|}{\delta_{n} \epsilon_{n}^{2}}+\frac{\left|\gamma_{n}-\gamma_{n-1}\right|}{\delta_{n} \epsilon_{n}^{2}}+\frac{\left|\lambda_{n} \alpha_{n}-\lambda_{n-1} \alpha_{n-1}\right|}{\delta_{n} \epsilon_{n}^{2}}+\frac{\left|\lambda_{n}-\lambda_{n-1}\right|}{\delta_{n} \epsilon_{n}^{2}}\right\}
\end{aligned}
$$

where $\sup _{n \geq 1}\left\{\left\|x_{n}-x_{n-1}\right\|+\widetilde{M}_{3}\right\} \leq \widetilde{M}_{4}$ for some $\widetilde{M}_{4}>0$. Utilizing Lemma 2.8, we conclude from conditions (C1)-(C6) and (C8)-(C9) that $\sum_{n=0}^{\infty} \epsilon_{n} \delta_{n}(1-\rho) \gamma=\infty$ and

$$
\lim _{n \rightarrow \infty} \frac{\left\|x_{n+1}-x_{n}\right\|}{\epsilon_{n}}=0
$$

So, as $\epsilon_{n} \rightarrow 0$, it follows that

$$
\lim _{n \rightarrow \infty}\left\|x_{n+1}-x_{n}\right\|=0
$$

Step 3. We prove that $\lim _{n \rightarrow \infty} \frac{\left\|x_{n}-u_{n}\right\|}{\epsilon_{n}}=0, \lim _{n \rightarrow \infty} \frac{\left\|x_{n}-v_{n}\right\|}{\epsilon_{n}}=0, \lim _{n \rightarrow \infty} \frac{\left\|v_{n}-t_{n}\right\|}{\epsilon_{n}}=0$ and $\lim _{n \rightarrow \infty} \frac{\left\|t_{n}-T t_{n}\right\|}{\epsilon_{n}}=0$.

Indeed, utilizing Lemmas 2.2 and 2.7(b), from (3.1), (3.4)-(3.5) and $\left(\gamma_{n}+\sigma_{n}\right) \xi \leq \gamma_{n}$, we deduce that

$$
\begin{aligned}
\left\|y_{n}-p\right\|^{2} & \\
= & \left\|\beta_{n} x_{n}+\gamma_{n} t_{n}+\sigma_{n} T t_{n}-p\right\|^{2} \\
& =\left\|\beta_{n}\left(x_{n}-p\right)+\left(1-\beta_{n}\right)\left(\frac{\gamma_{n} t_{n}+\sigma_{n} T t_{n}}{1-\beta_{n}}-p\right)\right\|^{2} \\
& =\beta_{n}\left\|x_{n}-p\right\|^{2}+\left(1-\beta_{n}\right)\left\|\frac{\gamma_{n} t_{n}+\sigma_{n} T t_{n}}{1-\beta_{n}}-p\right\|^{2}-\beta_{n}\left(1-\beta_{n}\right)\left\|\frac{\gamma_{n} t_{n}+\sigma_{n} T t_{n}}{1-\beta_{n}}-x_{n}\right\|^{2} \\
& =\beta_{n}\left\|x_{n}-p\right\|^{2}+\left(1-\beta_{n}\right)\left\|\frac{\gamma_{n}\left(t_{n}-p\right)+\sigma_{n}\left(T t_{n}-p\right)}{1-\beta_{n}}\right\|^{2}-\beta_{n}\left(1-\beta_{n}\right)\left\|\frac{y_{n}-x_{n}}{1-\beta_{n}}\right\|^{2} \\
& \leq \beta_{n}\left\|x_{n}-p\right\|^{2}+\left(1-\beta_{n}\right) \frac{\left(\gamma_{n}+\sigma_{n}\right)^{2}\left\|t_{n}-p\right\|^{2}}{\left(1-\beta_{n}\right)^{2}}-\frac{\beta_{n}}{1-\beta_{n}}\left\|y_{n}-x_{n}\right\|^{2} \\
& =\beta_{n}\left\|x_{n}-p\right\|^{2}+\left(1-\beta_{n}\right)\left\|t_{n}-p\right\|^{2}-\frac{\beta_{n}}{1-\beta_{n}}\left\|y_{n}-x_{n}\right\|^{2} \\
& \leq \beta_{n}\left\|x_{n}-p\right\|^{2}+\left(1-\beta_{n}\right)\left(\left\|x_{n}-p\right\|+\lambda_{n} \alpha_{n}\|p\|\right)^{2}-\frac{\beta_{n}}{1-\beta_{n}}\left\|y_{n}-x_{n}\right\|^{2} \\
& \leq \beta_{n}\left(\left\|x_{n}-p\right\|+\lambda_{n} \alpha_{n}\|p\|\right)^{2}+\left(1-\beta_{n}\right)\left(\left\|x_{n}-p\right\|+\lambda_{n} \alpha_{n}\|p\|\right)^{2}-\frac{\beta_{n}}{1-\beta_{n}}\left\|y_{n}-x_{n}\right\|^{2}
\end{aligned}
$$




$$
\begin{aligned}
& =\left(\left\|x_{n}-p\right\|+\lambda_{n} \alpha_{n}\|p\|\right)^{2}-\frac{\beta_{n}}{1-\beta_{n}}\left\|y_{n}-x_{n}\right\|^{2} \\
& \leq\left(\left\|x_{n}-p\right\|+\alpha_{n} b\|p\|\right)^{2}-\frac{\beta_{n}}{1-\beta_{n}}\left\|y_{n}-x_{n}\right\|^{2} .
\end{aligned}
$$

Observe that

$$
\begin{aligned}
\left\|\Delta_{n}^{k} x_{n}-p\right\|^{2} & =\left\|T_{r_{k, n}}^{\left(\Theta_{k}, \varphi_{k}\right)}\left(I-r_{k, n} A_{k}\right) \Delta_{n}^{k-1} x_{n}-T_{r_{k, n}}^{\left(\Theta_{k}, \varphi_{k}\right)}\left(I-r_{k, n} A_{k}\right) p\right\|^{2} \\
& \leq\left\|\left(I-r_{k, n} A_{k}\right) \Delta_{n}^{k-1} x_{n}-\left(I-r_{k, n} A_{k}\right) p\right\|^{2} \\
& \leq\left\|\Delta_{n}^{k-1} x_{n}-p\right\|^{2}+r_{k, n}\left(r_{k, n}-2 \mu_{k}\right)\left\|A_{k} \Delta_{n}^{k-1} x_{n}-A_{k} p\right\|^{2} \\
& \leq\left\|x_{n}-p\right\|^{2}+r_{k, n}\left(r_{k, n}-2 \mu_{k}\right)\left\|A_{k} \Delta_{n}^{k-1} x_{n}-A_{k} p\right\|^{2}
\end{aligned}
$$

and

$$
\begin{aligned}
\left\|\Lambda_{n}^{i} u_{n}-p\right\|^{2} & =\left\|J_{R_{i}, \lambda_{i, n}}\left(I-\lambda_{i, n} B_{i}\right) \Lambda_{n}^{i-1} u_{n}-J_{R_{i}, \lambda_{i, n}}\left(I-\lambda_{i, n} B_{i}\right) p\right\|^{2} \\
& \leq\left\|\left(I-\lambda_{i, n} B_{i}\right) \Lambda_{n}^{i-1} u_{n}-\left(I-\lambda_{i, n} B_{i}\right) p\right\|^{2} \\
& \leq\left\|\Lambda_{n}^{i-1} u_{n}-p\right\|^{2}+\lambda_{i, n}\left(\lambda_{i, n}-2 \eta_{i}\right)\left\|B_{i} \Lambda_{n}^{i-1} u_{n}-B_{i} p\right\|^{2} \\
& \leq\left\|u_{n}-p\right\|^{2}+\lambda_{i, n}\left(\lambda_{i, n}-2 \eta_{i}\right)\left\|B_{i} \Lambda_{n}^{i-1} u_{n}-B_{i} p\right\|^{2} \\
& \leq\left\|x_{n}-p\right\|^{2}+\lambda_{i, n}\left(\lambda_{i, n}-2 \eta_{i}\right)\left\|B_{i} \Lambda_{n}^{i-1} u_{n}-B_{i} p\right\|^{2}
\end{aligned}
$$

for $i \in\{1,2, \ldots, N\}$ and $k \in\{1,2, \ldots, M\}$. Combining (3.5), (3.15)-(3.17), we get

$$
\begin{aligned}
&\left\|y_{n}-p\right\|^{2} \\
& \leq \beta_{n}\left\|x_{n}-p\right\|^{2}+\left(1-\beta_{n}\right)\left\|t_{n}-p\right\|^{2}-\frac{\beta_{n}}{1-\beta_{n}}\left\|y_{n}-x_{n}\right\|^{2} \\
& \leq \beta_{n}\left\|x_{n}-p\right\|^{2}+\left(1-\beta_{n}\right)\left\|t_{n}-p\right\|^{2} \\
& \leq \beta_{n}\left\|x_{n}-p\right\|^{2}+\left(1-\beta_{n}\right)\left(\left\|v_{n}-p\right\|+\lambda_{n} \alpha_{n}\|p\|\right)^{2} \\
& \leq \beta_{n}\left\|x_{n}-p\right\|^{2}+\left(1-\beta_{n}\right)\left(\left\|v_{n}-p\right\|+\alpha_{n} b\|p\|\right)^{2} \\
&= \beta_{n}\left\|x_{n}-p\right\|^{2}+\left(1-\beta_{n}\right)\left[\left\|v_{n}-p\right\|^{2}+\alpha_{n} b\|p\|\left(2\left\|v_{n}-p\right\|+\alpha_{n} b\|p\|\right)\right] \\
& \leq \beta_{n}\left\|x_{n}-p\right\|^{2}+\left(1-\beta_{n}\right)\left\|v_{n}-p\right\|^{2}+\alpha_{n} b\|p\|\left(2\left\|v_{n}-p\right\|+\alpha_{n} b\|p\|\right) \\
& \leq \beta_{n}\left\|x_{n}-p\right\|^{2}+\left(1-\beta_{n}\right)\left\|\Lambda_{n}^{i} u_{n}-p\right\|^{2}+\alpha_{n} b\|p\|\left(2\left\|v_{n}-p\right\|+\alpha_{n} b\|p\|\right) \\
& \leq \beta_{n}\left\|x_{n}-p\right\|^{2}+\left(1-\beta_{n}\right)\left[\left\|u_{n}-p\right\|^{2}+\lambda_{i, n}\left(\lambda_{i, n}-2 \eta_{i}\right)\left\|B_{i} \Lambda_{n}^{i-1} u_{n}-B_{i} p\right\|^{2}\right] \\
&+\alpha_{n} b\|p\|\left(2\left\|v_{n}-p\right\|+\alpha_{n} b\|p\|\right) \\
& \leq \beta_{n}\left\|x_{n}-p\right\|^{2}+\left(1-\beta_{n}\right)\left[\left\|\Delta_{n}^{k} x_{n}-p\right\|^{2}+\lambda_{i, n}\left(\lambda_{i, n}-2 \eta_{i}\right)\left\|B_{i} \Lambda_{n}^{i-1} u_{n}-B_{i} p\right\|^{2}\right] \\
&+\alpha_{n} b\|p\|\left(2\left\|v_{n}-p\right\|+\alpha_{n} b\|p\|\right) \\
& \leq \beta_{n}\left\|x_{n}-p\right\|^{2}+\left(1-\beta_{n}\right)\left[\left\|x_{n}-p\right\|^{2}+r_{k, n}\left(r_{k, n}-2 \mu_{k}\right)\left\|A_{k} \Delta_{n}^{k-1} x_{n}-A_{k} p\right\|^{2}\right. \\
&\left.+\lambda_{i, n}\left(\lambda_{i, n}-2 \eta_{i}\right)\left\|B_{i} \Lambda_{n}^{i-1} u_{n}-B_{i} p\right\|^{2}\right]+\alpha_{n} b\|p\|\left(2\left\|v_{n}-p\right\|+\alpha_{n} b\|p\|\right) \\
&=\left\|x_{n}-p\right\|^{2}+\left(1-\beta_{n}\right)\left[r_{k, n}\left(r_{k, n}-2 \mu_{k}\right)\left\|A_{k} \Delta_{n}^{k-1} x_{n}-A_{k} p\right\|^{2}\right.
\end{aligned}
$$




$$
\begin{aligned}
& \left.+\lambda_{i, n}\left(\lambda_{i, n}-2 \eta_{i}\right)\left\|B_{i} \Lambda_{n}^{i-1} u_{n}-B_{i} p\right\|^{2}\right] \\
& +\alpha_{n} b\|p\|\left(2\left\|v_{n}-p\right\|+\alpha_{n} b\|p\|\right)
\end{aligned}
$$

which immediately leads to

$$
\begin{aligned}
& (1-d)\left[r_{k, n}\left(2 \mu_{k}-r_{k, n}\right) \frac{\left\|A_{k} \Delta_{n}^{k-1} x_{n}-A_{k} p\right\|^{2}}{\epsilon_{n}^{2}}+\lambda_{i, n}\left(2 \eta_{i}-\lambda_{i, n}\right) \frac{\left\|B_{i} \Lambda_{n}^{i-1} u_{n}-B_{i} p\right\|^{2}}{\epsilon_{n}^{2}}\right] \\
& \leq\left(1-\beta_{n}\right)\left[r_{k, n}\left(2 \mu_{k}-r_{k, n}\right) \frac{\left\|A_{k} \Delta_{n}^{k-1} x_{n}-A_{k} p\right\|^{2}}{\epsilon_{n}^{2}}+\lambda_{i, n}\left(2 \eta_{i}-\lambda_{i, n}\right) \frac{\left\|B_{i} \Lambda_{n}^{i-1} u_{n}-B_{i} p\right\|^{2}}{\epsilon_{n}^{2}}\right] \\
& \leq \frac{\left\|x_{n}-p\right\|^{2}-\left\|y_{n}-p\right\|^{2}}{\epsilon_{n}^{2}}+\frac{\alpha_{n}}{\epsilon_{n}^{2}} b\|p\|\left(2\left\|v_{n}-p\right\|+\alpha_{n} b\|p\|\right) \\
& \leq \frac{\left\|x_{n}-y_{n}\right\|}{\epsilon_{n}^{2}}\left(\left\|x_{n}-p\right\|+\left\|y_{n}-p\right\|\right)+\frac{\alpha_{n}}{\epsilon_{n}^{2}} b\|p\|\left(2\left\|v_{n}-p\right\|+\alpha_{n} b\|p\|\right)
\end{aligned}
$$

Since $\left\{\lambda_{i, n}\right\} \subset\left[a_{i}, b_{i}\right] \subset\left(0,2 \eta_{i}\right),\left\{r_{k, n}\right\} \subset\left[c_{k}, d_{k}\right] \subset\left(0,2 \mu_{k}\right), i \in\{1,2, \ldots, N\}, k \in\{1,2, \ldots, M\}$ and $\left\{v_{n}\right\},\left\{x_{n}\right\},\left\{y_{n}\right\}$ are bounded sequences, we obtain from $\left\|x_{n}-y_{n}\right\|+\alpha_{n}=o\left(\epsilon_{n}^{2}\right)$,

$$
\lim _{n \rightarrow \infty} \frac{\left\|A_{k} \Delta_{n}^{k-1} x_{n}-A_{k} p\right\|}{\epsilon_{n}}=0 \quad \text { and } \quad \lim _{n \rightarrow \infty} \frac{\left\|B_{i} \Lambda_{n}^{i-1} u_{n}-B_{i} p\right\|}{\epsilon_{n}}=0
$$

for all $k \in\{1,2, \ldots, M\}$ and $i \in\{1,2, \ldots, N\}$.

Furthermore, by Proposition 2.4(b) and Lemma 2.7(a), we have

$$
\begin{aligned}
&\left\|\Delta_{n}^{k} x_{n}-p\right\|^{2} \\
&=\left\|T_{r_{k, n}}^{\left(\Theta_{k}, \varphi_{k}\right)}\left(I-r_{k, n} A_{k}\right) \Delta_{n}^{k-1} x_{n}-T_{r_{k, n}}^{\left(\Theta_{k}, \varphi_{k}\right)}\left(I-r_{k, n} A_{k}\right) p\right\|^{2} \\
&\left.\leq \leq\left(I-r_{k, n} A_{k}\right) \Delta_{n}^{k-1} x_{n}-\left(I-r_{k, n} A_{k}\right) p, \Delta_{n}^{k} x_{n}-p\right\rangle \\
&=\frac{1}{2}\left(\left\|\left(I-r_{k, n} A_{k}\right) \Delta_{n}^{k-1} x_{n}-\left(I-r_{k, n} A_{k}\right) p\right\|^{2}+\left\|\Delta_{n}^{k} x_{n}-p\right\|^{2}\right. \\
&\left.\quad-\left\|\left(I-r_{k, n} A_{k}\right) \Delta_{n}^{k-1} x_{n}-\left(I-r_{k, n} A_{k}\right) p-\left(\Delta_{n}^{k} x_{n}-p\right)\right\|^{2}\right) \\
& \leq \frac{1}{2}\left(\left\|\Delta_{n}^{k-1} x_{n}-p\right\|^{2}+\left\|\Delta_{n}^{k} x_{n}-p\right\|^{2}\right. \\
&\left.\quad-\left\|\Delta_{n}^{k-1} x_{n}-\Delta_{n}^{k} x_{n}-r_{k, n}\left(A_{k} \Delta_{n}^{k-1} x_{n}-A_{k} p\right)\right\|^{2}\right),
\end{aligned}
$$

which implies that

$$
\begin{aligned}
& \left\|\Delta_{n}^{k} x_{n}-p\right\|^{2} \\
& \leq\left\|\Delta_{n}^{k-1} x_{n}-p\right\|^{2}-\left\|\Delta_{n}^{k-1} x_{n}-\Delta_{n}^{k} x_{n}-r_{k, n}\left(A_{k} \Delta_{n}^{k-1} x_{n}-A_{k} p\right)\right\|^{2} \\
& =\left\|\Delta_{n}^{k-1} x_{n}-p\right\|^{2}-\left\|\Delta_{n}^{k-1} x_{n}-\Delta_{n}^{k} x_{n}\right\|^{2}-r_{k, n}^{2}\left\|A_{k} \Delta_{n}^{k-1} x_{n}-A_{k} p\right\|^{2} \\
& \left.\quad+2 r_{k, n} \Delta_{n}^{k-1} x_{n}-\Delta_{n}^{k} x_{n}, A_{k} \Delta_{n}^{k-1} x_{n}-A_{k} p\right\rangle \\
& \leq\left\|\Delta_{n}^{k-1} x_{n}-p\right\|^{2}-\left\|\Delta_{n}^{k-1} x_{n}-\Delta_{n}^{k} x_{n}\right\|^{2}+2 r_{k, n}\left\|\Delta_{n}^{k-1} x_{n}-\Delta_{n}^{k} x_{n}\right\|\left\|A_{k} \Delta_{n}^{k-1} x_{n}-A_{k} p\right\| \\
& \leq\left\|x_{n}-p\right\|^{2}-\left\|\Delta_{n}^{k-1} x_{n}-\Delta_{n}^{k} x_{n}\right\|^{2} \\
& \quad+2 r_{k, n}\left\|\Delta_{n}^{k-1} x_{n}-\Delta_{n}^{k} x_{n}\right\|\left\|A_{k} \Delta_{n}^{k-1} x_{n}-A_{k} p\right\| .
\end{aligned}
$$


By Lemma 2.7(a) and Lemma 2.10, we obtain

$$
\begin{aligned}
&\left\|\Lambda_{n}^{i} u_{n}-p\right\|^{2} \\
&=\left\|J_{R_{i}, \lambda_{i, n}}\left(I-\lambda_{i, n} B_{i}\right) \Lambda_{n}^{i-1} u_{n}-J_{R_{i}, \lambda_{i, n}}\left(I-\lambda_{i, n} B_{i}\right) p\right\|^{2} \\
& \leq\left\langle\left(I-\lambda_{i, n} B_{i}\right) \Lambda_{n}^{i-1} u_{n}-\left(I-\lambda_{i, n} B_{i}\right) p, \Lambda_{n}^{i} u_{n}-p\right\rangle \\
&= \frac{1}{2}\left(\left\|\left(I-\lambda_{i, n} B_{i}\right) \Lambda_{n}^{i-1} u_{n}-\left(I-\lambda_{i, n} B_{i}\right) p\right\|^{2}+\left\|\Lambda_{n}^{i} u_{n}-p\right\|^{2}\right. \\
&\left.\quad-\left\|\left(I-\lambda_{i, n} B_{i}\right) \Lambda_{n}^{i-1} u_{n}-\left(I-\lambda_{i, n} B_{i}\right) p-\left(\Lambda_{n}^{i} u_{n}-p\right)\right\|^{2}\right) \\
& \leq \frac{1}{2}\left(\left\|\Lambda_{n}^{i-1} u_{n}-p\right\|^{2}+\left\|\Lambda_{n}^{i} u_{n}-p\right\|^{2}\right. \\
&\left.\quad-\left\|\Lambda_{n}^{i-1} u_{n}-\Lambda_{n}^{i} u_{n}-\lambda_{i, n}\left(B_{i} \Lambda_{n}^{i-1} u_{n}-B_{i} p\right)\right\|^{2}\right) \\
& \leq \frac{1}{2}\left(\left\|u_{n}-p\right\|^{2}+\left\|\Lambda_{n}^{i} u_{n}-p\right\|^{2}-\left\|\Lambda_{n}^{i-1} u_{n}-\Lambda_{n}^{i} u_{n}-\lambda_{i, n}\left(B_{i} \Lambda_{n}^{i-1} u_{n}-B_{i} p\right)\right\|^{2}\right) \\
& \leq \frac{1}{2}\left(\left\|x_{n}-p\right\|^{2}+\left\|\Lambda_{n}^{i} u_{n}-p\right\|^{2}-\left\|\Lambda_{n}^{i-1} u_{n}-\Lambda_{n}^{i} u_{n}-\lambda_{i, n}\left(B_{i} \Lambda_{n}^{i-1} u_{n}-B_{i} p\right)\right\|^{2}\right)
\end{aligned}
$$

which immediately leads to

$$
\begin{aligned}
\left\|\Lambda_{n}^{i} u_{n}-p\right\|^{2} \\
\leq\left\|x_{n}-p\right\|^{2}-\left\|\Lambda_{n}^{i-1} u_{n}-\Lambda_{n}^{i} u_{n}-\lambda_{i, n}\left(B_{i} \Lambda_{n}^{i-1} u_{n}-B_{i} p\right)\right\|^{2} \\
=\left\|x_{n}-p\right\|^{2}-\left\|\Lambda_{n}^{i-1} u_{n}-\Lambda_{n}^{k} u_{n}\right\|^{2}-\lambda_{i, n}^{2}\left\|B_{i} \Lambda_{n}^{i-1} u_{n}-B_{i} p\right\|^{2} \\
\quad+2 \lambda_{i, n}\left\langle\Lambda_{n}^{i-1} u_{n}-\Lambda_{n}^{i} u_{n}, B_{i} \Lambda_{n}^{i-1} u_{n}-B_{i} p\right\rangle \\
\leq\left\|x_{n}-p\right\|^{2}-\left\|\Lambda_{n}^{i-1} u_{n}-\Lambda_{n}^{i} u_{n}\right\|^{2} \\
\quad+2 \lambda_{i, n}\left\|\Lambda_{n}^{i-1} u_{n}-\Lambda_{n}^{i} u_{n}\right\|\left\|B_{i} \Lambda_{n}^{i-1} u_{n}-B_{i} p\right\| .
\end{aligned}
$$

Combining (3.15) and (3.21), we conclude that

$$
\begin{aligned}
\left\|y_{n}-p\right\|^{2} & \\
\leq & \beta_{n}\left\|x_{n}-p\right\|^{2}+\left(1-\beta_{n}\right)\left\|v_{n}-p\right\|^{2}+\alpha_{n} b\|p\|\left(2\left\|v_{n}-p\right\|+\alpha_{n} b\|p\|\right) \\
\leq & \beta_{n}\left\|x_{n}-p\right\|^{2}+\left(1-\beta_{n}\right)\left\|\Lambda_{n}^{i} u_{n}-p\right\|^{2}+\alpha_{n} b\|p\|\left(2\left\|v_{n}-p\right\|+\alpha_{n} b\|p\|\right) \\
\leq & \beta_{n}\left\|x_{n}-p\right\|^{2}+\left(1-\beta_{n}\right)\left[\left\|x_{n}-p\right\|^{2}-\left\|\Lambda_{n}^{i-1} u_{n}-\Lambda_{n}^{i} u_{n}\right\|^{2}\right. \\
& \left.\quad+2 \lambda_{i, n}\left\|\Lambda_{n}^{i-1} u_{n}-\Lambda_{n}^{i} u_{n}\right\|\left\|B_{i} \Lambda_{n}^{i-1} u_{n}-B_{i} p\right\|\right]+\alpha_{n} b\|p\|\left(2\left\|v_{n}-p\right\|+\alpha_{n} b\|p\|\right) \\
\leq & \left\|x_{n}-p\right\|^{2}-\left(1-\beta_{n}\right)\left\|\Lambda_{n}^{i-1} u_{n}-\Lambda_{n}^{i} u_{n}\right\|^{2} \\
& +2 \lambda_{i, n}\left\|\Lambda_{n}^{i-1} u_{n}-\Lambda_{n}^{i} u_{n}\right\|\left\|B_{i} \Lambda_{n}^{i-1} u_{n}-B_{i} p\right\|+\alpha_{n} b\|p\|\left(2\left\|v_{n}-p\right\|+\alpha_{n} b\|p\|\right),
\end{aligned}
$$

which yields

$$
\begin{aligned}
& (1-d) \frac{\left\|\Lambda_{n}^{i-1} u_{n}-\Lambda_{n}^{i} u_{n}\right\|^{2}}{\epsilon_{n}^{2}} \\
& \quad \leq\left(1-\beta_{n}\right)\left\|\Lambda_{n}^{i-1} u_{n}-\Lambda_{n}^{i} u_{n}\right\|^{2}
\end{aligned}
$$




$$
\begin{aligned}
\leq & \frac{\left\|x_{n}-p\right\|^{2}-\left\|y_{n}-p\right\|^{2}}{\epsilon_{n}^{2}}+2 \lambda_{i, n} \frac{\left\|\Lambda_{n}^{i-1} u_{n}-\Lambda_{n}^{i} u_{n}\right\|\left\|B_{i} \Lambda_{n}^{i-1} u_{n}-B_{i} p\right\|}{\epsilon_{n}^{2}} \\
& +\alpha_{n} b\|p\|\left(2\left\|v_{n}-p\right\|+\alpha_{n} b\|p\|\right) \\
\leq & \frac{\left\|x_{n}-y_{n}\right\|}{\epsilon_{n}^{2}}\left(\left\|x_{n}-p\right\|+\left\|y_{n}-p\right\|\right)+2 \lambda_{i, n} \frac{\left\|\Lambda_{n}^{i-1} u_{n}-\Lambda_{n}^{i} u_{n}\right\|}{\epsilon_{n}} \frac{\left\|B_{i} \Lambda_{n}^{i-1} u_{n}-B_{i} p\right\|}{\epsilon_{n}} \\
& +\frac{\alpha_{n}}{\epsilon_{n}^{2}} b\|p\|\left(2\left\|v_{n}-p\right\|+\alpha_{n} b\|p\|\right) .
\end{aligned}
$$

So, it follows from $\left\{\lambda_{i, n}\right\} \subset\left[a_{i}, b_{i}\right] \subset\left(0,2 \eta_{i}\right), i=1,2, \ldots, N$, that

$$
\begin{aligned}
(1-d) & \frac{\left\|\Lambda_{n}^{i-1} u_{n}-\Lambda_{n}^{i} u_{n}\right\|^{2}}{\epsilon_{n}^{2}} \\
\leq & \frac{\left\|x_{n}-y_{n}\right\|}{\epsilon_{n}^{2}}\left(\left\|x_{n}-p\right\|+\left\|y_{n}-p\right\|\right) \\
& +2 b_{i} \frac{\left\|\Lambda_{n}^{i-1} u_{n}-\Lambda_{n}^{i} u_{n}\right\|}{\epsilon_{n}} \frac{\left\|B_{i} \Lambda_{n}^{i-1} u_{n}-B_{i} p\right\|}{\epsilon_{n}} \\
& +\frac{\alpha_{n}}{\epsilon_{n}^{2}} b\|p\|\left(2\left\|v_{n}-p\right\|+\alpha_{n} b\|p\|\right) .
\end{aligned}
$$

Now we claim that

$$
\lim _{n \rightarrow \infty} \frac{\left\|\Lambda_{n}^{i-1} u_{n}-\Lambda_{n}^{i} u_{n}\right\|}{\epsilon_{n}}=0, \quad \forall i \in\{1,2, \ldots, N\} .
$$

As a matter of fact, it is easy to see that, for each $i \in\{1,2, \ldots, N\}$,

$$
\limsup _{n \rightarrow \infty} \frac{\left\|\Lambda_{n}^{i-1} u_{n}-\Lambda_{n}^{i} u_{n}\right\|}{\epsilon_{n}} \leq \infty .
$$

If $\lim \sup _{n \rightarrow \infty} \frac{\left\|\Lambda_{n}^{i-1} u_{n}-\Lambda_{n}^{i} u_{n}\right\|}{\epsilon_{n}}<\infty$, then from (3.22) and $\lim _{n \rightarrow \infty} \frac{\left\|B_{i} \Lambda_{n}^{i-1} u_{n}-B_{i} p\right\|}{\epsilon_{n}}=0$ (due to (3.9)), we have

$$
\begin{aligned}
(1-d) & \limsup _{n \rightarrow \infty} \frac{\left\|\Lambda_{n}^{i-1} u_{n}-\Lambda_{n}^{i} u_{n}\right\|^{2}}{\epsilon_{n}^{2}} \\
\leq & \limsup _{n \rightarrow \infty} \frac{\left\|x_{n}-y_{n}\right\|}{\epsilon_{n}^{2}}\left(\left\|x_{n}-p\right\|+\left\|y_{n}-p\right\|\right) \\
& +2 b_{i} \limsup _{n \rightarrow \infty} \frac{\left\|\Lambda_{n}^{i-1} u_{n}-\Lambda_{n}^{i} u_{n}\right\|}{\epsilon_{n}} \frac{\left\|B_{i} \Lambda_{n}^{i-1} u_{n}-B_{i} p\right\|}{\epsilon_{n}} \\
& +\limsup _{n \rightarrow \infty} \frac{\alpha_{n}}{\epsilon_{n}^{2}} b\|p\|\left(2\left\|v_{n}-p\right\|+\alpha_{n} b\|p\|\right) \\
\leq & \limsup _{n \rightarrow \infty} \frac{\left\|x_{n}-y_{n}\right\|}{\epsilon_{n}^{2}}\left(\left\|x_{n}-p\right\|+\left\|y_{n}-p\right\|\right) \\
& +2 b_{i} \limsup \sup _{n \rightarrow \infty} \frac{\left\|\Lambda_{n}^{i-1} u_{n}-\Lambda_{n}^{i} u_{n}\right\|}{\epsilon_{n}} \limsup _{n \rightarrow \infty} \frac{\left\|B_{i} \Lambda_{n}^{i-1} u_{n}-B_{i} p\right\|}{\epsilon_{n}} \\
& +\limsup _{n \rightarrow \infty} \frac{\alpha_{n}}{\epsilon_{n}^{2}} b\|p\|\left(2\left\|v_{n}-p\right\|+\alpha_{n} b\|p\|\right) \\
= & 0 .
\end{aligned}
$$


That is, $\lim _{n \rightarrow \infty} \frac{\left\|\Lambda_{n}^{i-1} u_{n}-\Lambda_{n}^{i} u_{n}\right\|}{\epsilon_{n}}=0$. If $\lim \sup _{n \rightarrow \infty} \frac{\left\|\Lambda_{n}^{i-1} u_{n}-\Lambda_{n}^{i} u_{n}\right\|}{\epsilon_{n}}=\infty$, then from (3.22), we have

$$
\begin{gathered}
(1-d) \frac{\left\|\Lambda_{n}^{i-1} u_{n}-\Lambda_{n}^{i} u_{n}\right\|}{\epsilon_{n}}\left[\frac{\left\|\Lambda_{n}^{i-1} u_{n}-\Lambda_{n}^{i} u_{n}\right\|}{\epsilon_{n}}-\frac{2 b_{i}}{1-d} \frac{\left\|B_{i} \Lambda_{n}^{i-1} u_{n}-B_{i} p\right\|}{\epsilon_{n}}\right] \\
\leq \frac{\left\|x_{n}-y_{n}\right\|}{\epsilon_{n}^{2}}\left(\left\|x_{n}-p\right\|+\left\|y_{n}-p\right\|\right)+\frac{\alpha_{n}}{\epsilon_{n}^{2}} b\|p\|\left(2\left\|v_{n}-p\right\|+\alpha_{n} b\|p\|\right) .
\end{gathered}
$$

Since $\lim _{n \rightarrow \infty} \frac{\left\|B_{i} \Lambda_{n}^{i-1} u_{n}-B_{i} p\right\|}{\epsilon_{n}}=0$ (due to (3.19)), it is easy to see that

$$
\limsup _{n \rightarrow \infty} \frac{\left\|\Lambda_{n}^{i-1} u_{n}-\Lambda_{n}^{i} u_{n}\right\|}{\epsilon_{n}}\left[\frac{\left\|\Lambda_{n}^{i-1} u_{n}-\Lambda_{n}^{i} u_{n}\right\|}{\epsilon_{n}}-\frac{2 b_{i}}{1-d} \frac{\left\|B_{i} \Lambda_{n}^{i-1} u_{n}-B_{i} p\right\|}{\epsilon_{n}}\right]=\infty .
$$

Thus, from (3.24), it follows that

$$
\begin{aligned}
\infty= & \limsup _{n \rightarrow \infty}(1-d) \frac{\left\|\Lambda_{n}^{i-1} u_{n}-\Lambda_{n}^{i} u_{n}\right\|}{\epsilon_{n}}\left[\frac{\left\|\Lambda_{n}^{i-1} u_{n}-\Lambda_{n}^{i} u_{n}\right\|}{\epsilon_{n}}-\frac{2 b_{i}}{1-d} \frac{\left\|B_{i} \Lambda_{n}^{i-1} u_{n}-B_{i} p\right\|}{\epsilon_{n}}\right] \\
\leq & \limsup _{n \rightarrow \infty} \frac{\left\|x_{n}-y_{n}\right\|}{\epsilon_{n}^{2}}\left(\left\|x_{n}-p\right\|+\left\|y_{n}-p\right\|\right) \\
& \quad+\limsup _{n \rightarrow \infty} \frac{\alpha_{n}}{\epsilon_{n}^{2}} b\|p\|\left(2\left\|v_{n}-p\right\|+\alpha_{n} b\|p\|\right) \\
= & 0,
\end{aligned}
$$

which leads to a contradiction. This shows that (3.23) holds.

Also, combining (3.3), (3.15), and (3.20), we deduce that

$$
\begin{aligned}
\left\|y_{n}-p\right\|^{2} \leq & \beta_{n}\left\|x_{n}-p\right\|^{2}+\left(1-\beta_{n}\right)\left\|v_{n}-p\right\|^{2}+\alpha_{n} b\|p\|\left(2\left\|v_{n}-p\right\|+\alpha_{n} b\|p\|\right) \\
\leq & \beta_{n}\left\|x_{n}-p\right\|^{2}+\left(1-\beta_{n}\right)\left\|u_{n}-p\right\|^{2}+\alpha_{n} b\|p\|\left(2\left\|v_{n}-p\right\|+\alpha_{n} b\|p\|\right) \\
\leq & \beta_{n}\left\|x_{n}-p\right\|^{2}+\left(1-\beta_{n}\right)\left\|\Delta_{n}^{k} x_{n}-p\right\|^{2}+\alpha_{n} b\|p\|\left(2\left\|v_{n}-p\right\|+\alpha_{n} b\|p\|\right) \\
\leq & \beta_{n}\left\|x_{n}-p\right\|^{2}+\left(1-\beta_{n}\right)\left[\left\|x_{n}-p\right\|^{2}-\left\|\Delta_{n}^{k-1} x_{n}-\Delta_{n}^{k} x_{n}\right\|^{2}\right. \\
& \left.+2 r_{k, n}\left\|\Delta_{n}^{k-1} x_{n}-\Delta_{n}^{k} x_{n}\right\|\left\|A_{k} \Delta_{n}^{k-1} x_{n}-A_{k} p\right\|\right] \\
& +\alpha_{n} b\|p\|\left(2\left\|v_{n}-p\right\|+\alpha_{n} b\|p\|\right) \\
\leq & \left\|x_{n}-p\right\|^{2}-\left(1-\beta_{n}\right)\left\|\Delta_{n}^{k-1} x_{n}-\Delta_{n}^{k} x_{n}\right\|^{2} \\
& +2 r_{k, n}\left\|\Delta_{n}^{k-1} x_{n}-\Delta_{n}^{k} x_{n}\right\|\left\|A_{k} \Delta_{n}^{k-1} x_{n}-A_{k} p\right\| \\
& +\alpha_{n} b\|p\|\left(2\left\|v_{n}-p\right\|+\alpha_{n} b\|p\|\right),
\end{aligned}
$$

which yields

$$
\begin{aligned}
& (1-d) \frac{\left\|\Delta_{n}^{k-1} x_{n}-\Delta_{n}^{k} x_{n}\right\|^{2}}{\epsilon_{n}^{2}} \\
& \leq\left(1-\beta_{n}\right) \frac{\left\|\Delta_{n}^{k-1} x_{n}-\Delta_{n}^{k} x_{n}\right\|^{2}}{\epsilon_{n}^{2}} \\
& \leq \frac{\left\|x_{n}-p\right\|^{2}-\left\|y_{n}-p\right\|^{2}}{\epsilon_{n}^{2}}+2 r_{k, n} \frac{\left\|\Delta_{n}^{k-1} x_{n}-\Delta_{n}^{k} x_{n}\right\|\left\|A_{k} \Delta_{n}^{k-1} x_{n}-A_{k} p\right\|}{\epsilon_{n}^{2}}
\end{aligned}
$$




$$
\begin{aligned}
& +\frac{\alpha_{n}}{\epsilon_{n}^{2}} b\|p\|\left(2\left\|v_{n}-p\right\|+\alpha_{n} b\|p\|\right) \\
\leq & \frac{\left\|x_{n}-y_{n}\right\|}{\epsilon_{n}^{2}}\left(\left\|x_{n}-p\right\|+\left\|y_{n}-p\right\|\right)+2 r_{k, n} \frac{\left\|\Delta_{n}^{k-1} x_{n}-\Delta_{n}^{k} x_{n}\right\|}{\epsilon_{n}} \frac{\left\|A_{k} \Delta_{n}^{k-1} x_{n}-A_{k} p\right\|}{\epsilon_{n}} \\
& +\frac{\alpha_{n}}{\epsilon_{n}^{2}} b\|p\|\left(2\left\|v_{n}-p\right\|+\alpha_{n} b\|p\|\right) .
\end{aligned}
$$

So, it follows from $\left\{r_{k, n}\right\} \subset\left[c_{k}, d_{k}\right] \subset\left(0,2 \mu_{k}\right), k=1,2, \ldots, M$, that

$$
\begin{aligned}
(1-d) & \frac{\left\|\Delta_{n}^{k-1} x_{n}-\Delta_{n}^{k} x_{n}\right\|^{2}}{\epsilon_{n}^{2}} \\
\leq & \frac{\left\|x_{n}-y_{n}\right\|}{\epsilon_{n}^{2}}\left(\left\|x_{n}-p\right\|+\left\|y_{n}-p\right\|\right) \\
& +2 d_{k} \frac{\left\|\Delta_{n}^{k-1} x_{n}-\Delta_{n}^{k} x_{n}\right\|}{\epsilon_{n}} \frac{\left\|A_{k} \Delta_{n}^{k-1} x_{n}-A_{k} p\right\|}{\epsilon_{n}} \\
& +\frac{\alpha_{n}}{\epsilon_{n}^{2}} b\|p\|\left(2\left\|v_{n}-p\right\|+\alpha_{n} b\|p\|\right) .
\end{aligned}
$$

Next, we claim that

$$
\lim _{n \rightarrow \infty} \frac{\left\|\Delta_{n}^{k-1} x_{n}-\Delta_{n}^{k} x_{n}\right\|}{\epsilon_{n}}=0, \quad \forall k \in\{1,2, \ldots, M\}
$$

As a matter of fact, it is easy to see that, for each $k \in\{1,2, \ldots, M\}$,

$$
\limsup _{n \rightarrow \infty} \frac{\left\|\Delta_{n}^{k-1} x_{n}-\Delta_{n}^{k} x_{n}\right\|}{\epsilon_{n}} \leq \infty
$$

If $\lim \sup _{n \rightarrow \infty} \frac{\left\|\Delta_{n}^{k-1} x_{n}-\Delta_{n}^{k} x_{n}\right\|}{\epsilon_{n}}<\infty$, then from (3.25) and $\lim \sup _{n \rightarrow \infty} \frac{\left\|A_{k} \Delta_{n}^{k-1} x_{n}-A_{k} p\right\|}{\epsilon_{n}}=0$ (due to (3.19)), we have

$$
\begin{aligned}
& \limsup _{n \rightarrow \infty}(1-d) \frac{\left\|\Delta_{n}^{k-1} x_{n}-\Delta_{n}^{k} x_{n}\right\|^{2}}{\epsilon_{n}^{2}} \\
& \leq \limsup _{n \rightarrow \infty} \frac{\left\|x_{n}-y_{n}\right\|}{\epsilon_{n}^{2}}\left(\left\|x_{n}-p\right\|+\left\|y_{n}-p\right\|\right) \\
& \quad+2 d_{k} \limsup _{n \rightarrow \infty} \frac{\left\|\Delta_{n}^{k-1} x_{n}-\Delta_{n}^{k} x_{n}\right\|}{\epsilon_{n}} \frac{\left\|A_{k} \Delta_{n}^{k-1} x_{n}-A_{k} p\right\|}{\epsilon_{n}} \\
& \quad+\limsup _{n \rightarrow \infty} \frac{\alpha_{n}}{\epsilon_{n}^{2}} b\|p\|\left(2\left\|v_{n}-p\right\|+\alpha_{n} b\|p\|\right) \\
& \leq \limsup _{n \rightarrow \infty} \frac{\left\|x_{n}-y_{n}\right\|}{\epsilon_{n}^{2}}\left(\left\|x_{n}-p\right\|+\left\|y_{n}-p\right\|\right) \\
& \quad+2 d_{k} \limsup \sup _{n \rightarrow \infty} \frac{\left\|\Delta_{n}^{k-1} x_{n}-\Delta_{n}^{k} x_{n}\right\|}{\epsilon_{n}} \limsup _{n \rightarrow \infty} \frac{\left\|A_{k} \Delta_{n}^{k-1} x_{n}-A_{k} p\right\|}{\epsilon_{n}} \\
& \quad+\limsup _{n \rightarrow \infty} \frac{\alpha_{n}}{\epsilon_{n}^{2}} b\|p\|\left(2\left\|v_{n}-p\right\|+\alpha_{n} b\|p\|\right) \\
& =0 .
\end{aligned}
$$


That is, $\lim _{n \rightarrow \infty} \frac{\left\|\Delta_{n}^{k-1} x_{n}-\Delta_{n}^{k} x_{n}\right\|}{\epsilon_{n}}=0$. If $\limsup _{n \rightarrow \infty} \frac{\left\|\Delta_{n}^{k-1} x_{n}-\Delta_{n}^{k} x_{n}\right\|}{\epsilon_{n}}=\infty$, then from (3.25), we have

$$
\begin{gathered}
(1-d) \frac{\left\|\Delta_{n}^{k-1} x_{n}-\Delta_{n}^{k} x_{n}\right\|}{\epsilon_{n}}\left[\frac{\left\|\Delta_{n}^{k-1} x_{n}-\Delta_{n}^{k} x_{n}\right\|}{\epsilon_{n}}-\frac{\left\|A_{k} \Delta_{n}^{k-1} x_{n}-A_{k} p\right\|}{\epsilon_{n}}\right] \\
\leq \frac{\left\|x_{n}-y_{n}\right\|}{\epsilon_{n}^{2}}\left(\left\|x_{n}-p\right\|+\left\|y_{n}-p\right\|\right)+\frac{\alpha_{n}}{\epsilon_{n}^{2}} b\|p\|\left(2\left\|v_{n}-p\right\|+\alpha_{n} b\|p\|\right) .
\end{gathered}
$$

Since $\lim _{n \rightarrow \infty} \frac{\left\|A_{k} \Delta_{n}^{k-1} x_{n}-A_{k} p\right\|}{\epsilon_{n}}=0$ (due to (3.19)), it is easy to see that

$$
\limsup _{n \rightarrow \infty} \frac{\left\|\Delta_{n}^{k-1} x_{n}-\Delta_{n}^{k} x_{n}\right\|}{\epsilon_{n}}\left[\frac{\left\|\Delta_{n}^{k-1} x_{n}-\Delta_{n}^{k} x_{n}\right\|}{\epsilon_{n}}-\frac{\left\|A_{k} \Delta_{n}^{k-1} x_{n}-A_{k} p\right\|}{\epsilon_{n}}\right]=\infty .
$$

Consequently, from (3.27), it follows that

$$
\begin{aligned}
\infty= & \limsup _{n \rightarrow \infty}(1-d) \frac{\left\|\Delta_{n}^{k-1} x_{n}-\Delta_{n}^{k} x_{n}\right\|}{\epsilon_{n}}\left[\frac{\left\|\Delta_{n}^{k-1} x_{n}-\Delta_{n}^{k} x_{n}\right\|}{\epsilon_{n}}-\frac{\left\|A_{k} \Delta_{n}^{k-1} x_{n}-A_{k} p\right\|}{\epsilon_{n}}\right] \\
\leq & \limsup _{n \rightarrow \infty} \frac{\left\|x_{n}-y_{n}\right\|}{\epsilon_{n}^{2}}\left(\left\|x_{n}-p\right\|+\left\|y_{n}-p\right\|\right) \\
& +\limsup _{n \rightarrow \infty} \frac{\alpha_{n}}{\epsilon_{n}^{2}} b\|p\|\left(2\left\|v_{n}-p\right\|+\alpha_{n} b\|p\|\right) \\
= & 0,
\end{aligned}
$$

which leads to a contradiction. This shows that (3.26) is valid. Therefore, from (3.23) and (3.26), we get

$$
\begin{aligned}
\frac{\left\|x_{n}-u_{n}\right\|}{\epsilon_{n}}= & \frac{\left\|\Delta_{n}^{0} x_{n}-\Delta_{n}^{M} x_{n}\right\|}{\epsilon_{n}} \\
\leq & \frac{\left\|\Delta_{n}^{0} x_{n}-\Delta_{n}^{1} x_{n}\right\|}{\epsilon_{n}}+\frac{\left\|\Delta_{n}^{1} x_{n}-\Delta_{n}^{2} x_{n}\right\|}{\epsilon_{n}} \\
& +\cdots+\frac{\left\|\Delta_{n}^{M-1} x_{n}-\Delta_{n}^{M} x_{n}\right\|}{\epsilon_{n}} \\
\rightarrow & 0 \quad \text { as } n \rightarrow \infty
\end{aligned}
$$

and

$$
\begin{aligned}
\frac{\left\|u_{n}-v_{n}\right\|}{\epsilon_{n}}= & \frac{\left\|\Lambda_{n}^{0} u_{n}-\Lambda_{n}^{N} u_{n}\right\|}{\epsilon_{n}} \\
\leq & \frac{\left\|\Lambda_{n}^{0} u_{n}-\Lambda_{n}^{1} u_{n}\right\|}{\epsilon_{n}}+\frac{\left\|\Lambda_{n}^{1} u_{n}-\Lambda_{n}^{2} u_{n}\right\|}{\epsilon_{n}} \\
& +\cdots+\frac{\left\|\Lambda_{n}^{N-1} u_{n}-\Lambda_{n}^{N} u_{n}\right\|}{\epsilon_{n}} \\
\rightarrow & 0 \quad \text { as } n \rightarrow \infty,
\end{aligned}
$$

respectively. Thus, from (3.28) and (3.29), we obtain

$$
\frac{\left\|x_{n}-v_{n}\right\|}{\epsilon_{n}} \leq \frac{\left\|x_{n}-u_{n}\right\|}{\epsilon_{n}}+\frac{\left\|u_{n}-v_{n}\right\|}{\epsilon_{n}} \rightarrow 0 \quad \text { as } n \rightarrow \infty .
$$


On the other hand, note that $\Gamma=\operatorname{VI}(C, \nabla f)$. Then, utilizing Lemma 2.6 and the $\frac{1}{\|A\|^{2}}$ inverse strong monotonicity of $\nabla f$, we deduce from (2.1) that

$$
\begin{aligned}
\left\|t_{n}-p\right\|^{2} \leq & \left\|\left(I-\lambda_{n} \nabla f_{\alpha_{n}}\right) v_{n}-\left(I-\lambda_{n} \nabla f\right) p\right\|^{2} \\
= & \left\|v_{n}-p-\lambda_{n}\left(\nabla f\left(v_{n}\right)-\nabla f(p)\right)-\lambda_{n} \alpha_{n} v_{n}\right\|^{2} \\
\leq & \left\|v_{n}-p-\lambda_{n}\left(\nabla f\left(v_{n}\right)-\nabla f(p)\right)\right\|^{2} \\
& \quad-2 \lambda_{n} \alpha_{n}\left(v_{n},\left(I-\lambda_{n} \nabla f_{\alpha_{n}}\right) v_{n}-\left(I-\lambda_{n} \nabla f\right) p\right\rangle \\
\leq & \left\|v_{n}-p\right\|^{2}+\lambda_{n}\left(\lambda_{n}-\frac{2}{\|A\|^{2}}\right)\left\|\nabla f\left(v_{n}\right)-\nabla f(p)\right\|^{2} \\
& +2 \alpha_{n} b\left\|v_{n}\right\|\left\|v_{n}-p-\lambda_{n}\left(\nabla f_{\alpha_{n}}\left(v_{n}\right)-\nabla f(p)\right)\right\| .
\end{aligned}
$$

Combining (3.4), (3.15), and (3.31), we obtain

$$
\begin{aligned}
\left\|y_{n}-p\right\|^{2} \leq & \beta_{n}\left\|x_{n}-p\right\|^{2}+\left(1-\beta_{n}\right)\left\|t_{n}-p\right\|^{2} \\
\leq & \beta_{n}\left\|x_{n}-p\right\|^{2}+\left(1-\beta_{n}\right)\left[\left\|v_{n}-p\right\|^{2}+\lambda_{n}\left(\lambda_{n}-\frac{2}{\|A\|^{2}}\right)\left\|\nabla f\left(v_{n}\right)-\nabla f(p)\right\|^{2}\right. \\
& \left.+2 \alpha_{n} b\left\|v_{n}\right\|\left\|v_{n}-p-\lambda_{n}\left(\nabla f_{\alpha_{n}}\left(v_{n}\right)-\nabla f(p)\right)\right\|\right] \\
\leq & \beta_{n}\left\|x_{n}-p\right\|^{2}+\left(1-\beta_{n}\right)\left[\left\|x_{n}-p\right\|^{2}+\lambda_{n}\left(\lambda_{n}-\frac{2}{\|A\|^{2}}\right)\left\|\nabla f\left(v_{n}\right)-\nabla f(p)\right\|^{2}\right. \\
& \left.+2 \alpha_{n} b\left\|v_{n}\right\|\left\|v_{n}-p-\lambda_{n}\left(\nabla f_{\alpha_{n}}\left(v_{n}\right)-\nabla f(p)\right)\right\|\right] \\
\leq & \left\|x_{n}-p\right\|^{2}+\left(1-\beta_{n}\right) \lambda_{n}\left(\lambda_{n}-\frac{2}{\|A\|^{2}}\right)\left\|\nabla f\left(v_{n}\right)-\nabla f(p)\right\|^{2} \\
& +2 \alpha_{n} b\left\|v_{n}\right\|\left\|v_{n}-p-\lambda_{n}\left(\nabla f_{\alpha_{n}}\left(v_{n}\right)-\nabla f(p)\right)\right\|,
\end{aligned}
$$

which together with $\left\{\lambda_{n}\right\} \subset[a, b] \subset\left(0, \frac{2}{\|A\|^{2}}\right)$ and $\left\{\beta_{n}\right\} \subset[c, d] \subset(0,1)$ leads to

$$
\begin{aligned}
(1-d) a\left(\frac{2}{\|A\|^{2}}-b\right) \frac{\left\|\nabla f\left(v_{n}\right)-\nabla f(p)\right\|^{2}}{\epsilon_{n}^{2}} \\
\leq\left(1-\beta_{n}\right) \lambda_{n}\left(\frac{2}{\|A\|^{2}}-\lambda_{n}\right) \frac{\left\|\nabla f\left(v_{n}\right)-\nabla f(p)\right\|^{2}}{\epsilon_{n}^{2}} \\
\leq \frac{\left\|x_{n}-p\right\|^{2}-\left\|y_{n}-p\right\|^{2}}{\epsilon_{n}^{2}}+2 \frac{\alpha_{n}}{\epsilon_{n}^{2}} b\left\|v_{n}\right\|\left\|v_{n}-p-\lambda_{n}\left(\nabla f_{\alpha_{n}}\left(v_{n}\right)-\nabla f(p)\right)\right\| \\
\leq \frac{\left\|x_{n}-y_{n}\right\|}{\epsilon_{n}^{2}}\left(\left\|x_{n}-p\right\|+\left\|y_{n}-p\right\|\right) \\
\quad+2 \frac{\alpha_{n}}{\epsilon_{n}^{2}} b\left\|v_{n}\right\|\left\|v_{n}-p-\lambda_{n}\left(\nabla f_{\alpha_{n}}\left(v_{n}\right)-\nabla f(p)\right)\right\| .
\end{aligned}
$$

Since $\left\{v_{n}\right\},\left\{x_{n}\right\}$, and $\left\{y_{n}\right\}$ are bounded sequences, we deduce from $\left\|x_{n}-y_{n}\right\|+\alpha_{n}=o\left(\epsilon_{n}^{2}\right)$ that

$$
\lim _{n \rightarrow \infty} \frac{\left\|\nabla f\left(v_{n}\right)-\nabla f(p)\right\|}{\epsilon_{n}}=0 .
$$


So, it is clear that

$$
\lim _{n \rightarrow \infty} \frac{\left\|\nabla f_{\alpha_{n}}\left(v_{n}\right)-\nabla f(p)\right\|}{\epsilon_{n}}=0 .
$$

Again, utilizing Proposition 2.3(c), from $t_{n}=P_{C}\left(I-\lambda_{n} \nabla f_{\alpha_{n}}\right) v_{n}$ and $p=P_{C}\left(I-\lambda_{n} \nabla f\right) p$, we get

$$
\begin{aligned}
\left\|t_{n}-p\right\|^{2}= & \left\|P_{C}\left(I-\lambda_{n} \nabla f_{\alpha_{n}}\right) v_{n}-P_{C}\left(I-\lambda_{n} \nabla f\right) p\right\|^{2} \\
\leq & \left\langle\left(I-\lambda_{n} \nabla f_{\alpha_{n}}\right) v_{n}-\left(I-\lambda_{n} \nabla f\right) p, t_{n}-p\right\rangle \\
= & \frac{1}{2}\left(\left\|\left(I-\lambda_{n} \nabla f_{\alpha_{n}}\right) v_{n}-\left(I-\lambda_{n} \nabla f\right) p\right\|^{2}+\left\|t_{n}-p\right\|^{2}\right. \\
& \left.-\left\|\left(I-\lambda_{n} \nabla f_{\alpha_{n}}\right) v_{n}-\left(I-\lambda_{n} \nabla f\right) p-\left(t_{n}-p\right)\right\|^{2}\right) \\
= & \frac{1}{2}\left(\left\|\left(I-\lambda_{n} \nabla f_{\alpha_{n}}\right) v_{n}-\left(I-\lambda_{n} \nabla f_{\alpha_{n}}\right) p-\lambda_{n} \alpha_{n} p\right\|^{2}+\left\|t_{n}-p\right\|^{2}\right. \\
& \left.-\left\|\left(I-\lambda_{n} \nabla f_{\alpha_{n}}\right) v_{n}-\left(I-\lambda_{n} \nabla f\right) p-\left(t_{n}-p\right)\right\|^{2}\right) \\
= & \frac{1}{2}\left(\left\|\left(I-\lambda_{n} \nabla f_{\alpha_{n}}\right) v_{n}-\left(I-\lambda_{n} \nabla f_{\alpha_{n}}\right) p\right\|^{2}-2 \lambda_{n} \alpha_{n}\left\langle p,\left(I-\lambda_{n} \nabla f_{\alpha_{n}}\right) v_{n}\right.\right. \\
& \left.-\left(I-\lambda_{n} \nabla f\right) p\right\rangle \\
& \left.+\left\|t_{n}-p\right\|^{2}-\left\|\left(I-\lambda_{n} \nabla f_{\alpha_{n}}\right) v_{n}-\left(I-\lambda_{n} \nabla f\right) p-\left(t_{n}-p\right)\right\|^{2}\right) \\
\leq & \frac{1}{2}\left(\left\|v_{n}-p\right\|^{2}-2 \lambda_{n} \alpha_{n}\left\langle p,\left(I-\lambda_{n} \nabla f_{\alpha_{n}}\right) v_{n}-\left(I-\lambda_{n} \nabla f\right) p\right\rangle+\left\|t_{n}-p\right\|^{2}\right. \\
& \left.-\left\|\left(I-\lambda_{n} \nabla f_{\alpha_{n}}\right) v_{n}-\left(I-\lambda_{n} \nabla f\right) p-\left(t_{n}-p\right)\right\|^{2}\right) \\
\leq & \frac{1}{2}\left(\left\|v_{n}-p\right\|^{2}+2 \lambda_{n} \alpha_{n}\|p\|\left\|\left(I-\lambda_{n} \nabla f_{\alpha_{n}}\right) v_{n}-\left(I-\lambda_{n} \nabla f\right) p\right\|+\left\|t_{n}-p\right\|^{2}\right. \\
& \left.-\left\|v_{n}-t_{n}-\lambda_{n}\left(\nabla f_{\alpha_{n}}\left(v_{n}\right)-\nabla f(p)\right)\right\|^{2}\right),
\end{aligned}
$$

which immediately leads to

$$
\begin{aligned}
\left\|t_{n}-p\right\|^{2} \leq & \left\|v_{n}-p\right\|^{2}+2 \lambda_{n} \alpha_{n}\|p\|\left\|\left(I-\lambda_{n} \nabla f_{\alpha_{n}}\right) v_{n}-\left(I-\lambda_{n} \nabla f\right) p\right\| \\
& -\left\|v_{n}-t_{n}-\lambda_{n}\left(\nabla f_{\alpha_{n}}\left(v_{n}\right)-\nabla f(p)\right)\right\|^{2}
\end{aligned}
$$

Combining (3.4), (3.15), and (3.33), we obtain

$$
\begin{aligned}
\left\|y_{n}-p\right\|^{2} \leq & \beta_{n}\left\|x_{n}-p\right\|^{2}+\left(1-\beta_{n}\right)\left\|t_{n}-p\right\|^{2} \\
\leq & \beta_{n}\left\|x_{n}-p\right\|^{2}+\left(1-\beta_{n}\right)\left[\left\|v_{n}-p\right\|^{2}\right. \\
& +2 \lambda_{n} \alpha_{n}\|p\|\left\|\left(I-\lambda_{n} \nabla f_{\alpha_{n}}\right) v_{n}-\left(I-\lambda_{n} \nabla f\right) p\right\| \\
& \left.-\left\|v_{n}-t_{n}-\lambda_{n}\left(\nabla f_{\alpha_{n}}\left(v_{n}\right)-\nabla f(p)\right)\right\|^{2}\right] \\
\leq & \beta_{n}\left\|x_{n}-p\right\|^{2}+\left(1-\beta_{n}\right)\left[\left\|x_{n}-p\right\|^{2}\right. \\
& +2 \lambda_{n} \alpha_{n}\|p\|\left\|\left(I-\lambda_{n} \nabla f_{\alpha_{n}}\right) v_{n}-\left(I-\lambda_{n} \nabla f\right) p\right\| \\
& \left.-\left\|v_{n}-t_{n}-\lambda_{n}\left(\nabla f_{\alpha_{n}}\left(v_{n}\right)-\nabla f(p)\right)\right\|^{2}\right]
\end{aligned}
$$




$$
\begin{aligned}
\leq & \left\|x_{n}-p\right\|^{2}+2 \lambda_{n} \alpha_{n}\|p\|\left\|\left(I-\lambda_{n} \nabla f_{\alpha_{n}}\right) v_{n}-\left(I-\lambda_{n} \nabla f\right) p\right\| \\
& -\left(1-\beta_{n}\right)\left\|v_{n}-t_{n}-\lambda_{n}\left(\nabla f_{\alpha_{n}}\left(v_{n}\right)-\nabla f(p)\right)\right\|^{2},
\end{aligned}
$$

which immediately yields

$$
\begin{aligned}
& (1-d) \frac{\left\|v_{n}-t_{n}-\lambda_{n}\left(\nabla f_{\alpha_{n}}\left(v_{n}\right)-\nabla f(p)\right)\right\|^{2}}{\epsilon_{n}^{2}} \\
& \quad \leq\left(1-\beta_{n}\right) \frac{\left\|v_{n}-t_{n}-\lambda_{n}\left(\nabla f_{\alpha_{n}}\left(v_{n}\right)-\nabla f(p)\right)\right\|^{2}}{\epsilon_{n}^{2}} \\
& \quad \leq \frac{\left\|x_{n}-p\right\|^{2}-\left\|y_{n}-p\right\|^{2}}{\epsilon_{n}^{2}}+2 \lambda_{n} \frac{\alpha_{n}}{\epsilon_{n}^{2}}\|p\|\left\|\left(I-\lambda_{n} \nabla f_{\alpha_{n}}\right) v_{n}-\left(I-\lambda_{n} \nabla f\right) p\right\| \\
& \leq \frac{\left\|x_{n}-y_{n}\right\|}{\epsilon_{n}^{2}}\left(\left\|x_{n}-p\right\|+\left\|y_{n}-p\right\|\right)+2 \frac{\alpha_{n}}{\epsilon_{n}^{2}} b\|p\|\left\|\left(I-\lambda_{n} \nabla f_{\alpha_{n}}\right) \nu_{n}-\left(I-\lambda_{n} \nabla f\right) p\right\| .
\end{aligned}
$$

Since $\left\{v_{n}\right\},\left\{x_{n}\right\}$, and $\left\{y_{n}\right\}$ are bounded sequences, we deduce from $\left\|x_{n}-y_{n}\right\|+\alpha_{n}=o\left(\epsilon_{n}^{2}\right)$ that

$$
\lim _{n \rightarrow \infty} \frac{\left\|v_{n}-t_{n}-\lambda_{n}\left(\nabla f_{\alpha_{n}}\left(v_{n}\right)-\nabla f(p)\right)\right\|}{\epsilon_{n}}=0
$$

Observe that

$$
\frac{\left\|v_{n}-t_{n}\right\|}{\epsilon_{n}} \leq \frac{\left\|v_{n}-t_{n}-\lambda_{n}\left(\nabla f_{\alpha_{n}}\left(v_{n}\right)-\nabla f(p)\right)\right\|}{\epsilon_{n}}+\frac{\lambda_{n}\left\|\nabla f_{\alpha_{n}}\left(v_{n}\right)-\nabla f(p)\right\|}{\epsilon_{n}} .
$$

Thus, from (3.32) and (3.34), we have

$$
\lim _{n \rightarrow \infty} \frac{\left\|v_{n}-t_{n}\right\|}{\epsilon_{n}}=0 .
$$

Taking into account that $\frac{\left\|x_{n}-t_{n}\right\|}{\epsilon_{n}} \leq \frac{\left\|x_{n}-v_{n}\right\|}{\epsilon_{n}}+\frac{\left\|v_{n}-t_{n}\right\|}{\epsilon_{n}}$, from (3.30) and (3.35), we get

$$
\lim _{n \rightarrow \infty} \frac{\left\|x_{n}-t_{n}\right\|}{\epsilon_{n}}=0 .
$$

Utilizing the relation $y_{n}-x_{n}=\gamma_{n}\left(t_{n}-x_{n}\right)+\sigma_{n}\left(T t_{n}-x_{n}\right)$, we have

$$
\begin{aligned}
\frac{\left\|\sigma_{n}\left(T t_{n}-t_{n}\right)\right\|}{\epsilon_{n}} & =\frac{\left\|\sigma_{n}\left(T t_{n}-x_{n}\right)-\sigma_{n}\left(t_{n}-x_{n}\right)\right\|}{\epsilon_{n}} \\
& =\frac{\left\|y_{n}-x_{n}-\gamma_{n}\left(t_{n}-x_{n}\right)-\sigma_{n}\left(t_{n}-x_{n}\right)\right\|}{\epsilon_{n}} \\
& =\frac{\left\|y_{n}-x_{n}-\left(1-\beta_{n}\right)\left(t_{n}-x_{n}\right)\right\|}{\epsilon_{n}} \\
& \leq \frac{\left\|y_{n}-x_{n}\right\|}{\epsilon_{n}}+\left(1-\beta_{n}\right) \frac{\left\|t_{n}-x_{n}\right\|}{\epsilon_{n}} \\
& \leq \frac{\left\|y_{n}-x_{n}\right\|}{\epsilon_{n}}+\frac{\left\|t_{n}-x_{n}\right\|}{\epsilon_{n}},
\end{aligned}
$$


which together with (3.36) and $\left\|x_{n}-y_{n}\right\|=o\left(\epsilon_{n}^{2}\right)$, implies that

$$
\lim _{n \rightarrow \infty} \frac{\left\|\sigma_{n}\left(T t_{n}-t_{n}\right)\right\|}{\epsilon_{n}}=0
$$

Since $\liminf _{n \rightarrow \infty} \sigma_{n}>0$, we obtain

$$
\lim _{n \rightarrow \infty} \frac{\left\|t_{n}-T t_{n}\right\|}{\epsilon_{n}}=0 .
$$

Step 4. We prove that $\omega_{w}\left(x_{n}\right) \subset \Omega$.

Indeed, since $H$ is reflexive and $\left\{x_{n}\right\}$ is bounded, there exists at least a weak convergence subsequence of $\left\{x_{n}\right\}$. Hence, $\omega_{w}\left(x_{n}\right) \neq \emptyset$. Now, take an arbitrary $w \in \omega_{w}\left(x_{n}\right)$. Then there exists a subsequence $\left\{x_{n_{i}}\right\}$ of $\left\{x_{n}\right\}$ such that $x_{n_{i}} \rightarrow w$. From (3.23), (3.26), (3.28), (3.30) and (3.36), we have $u_{n_{i}} \rightarrow w, v_{n_{i}} \rightarrow w, t_{n_{i}} \rightarrow w, \Lambda_{n_{i}}^{m} u_{n_{i}} \rightarrow w$ and $\Delta_{n_{i}}^{k} x_{n_{i}} \rightarrow w$, where $m \in$ $\{1,2, \ldots, N\}$ and $k \in\{1,2, \ldots, M\}$. Utilizing Lemma 2.1(b), we deduce from $t_{n_{i}} \rightarrow w$ and (3.37) that $w \in \operatorname{Fix}(T)$.

Next, we prove that $w \in \bigcap_{m=1}^{N} \mathrm{I}\left(B_{m}, R_{m}\right)$. As a matter of fact, since $B_{m}$ is $\eta_{m}$-inverse strongly monotone, $B_{m}$ is a monotone and Lipschitz-continuous mapping. It follows from Lemma 2.13 that $R_{m}+B_{m}$ is maximal monotone. Let $(v, g) \in G\left(R_{m}+B_{m}\right)$, that is, $g-B_{m} v \in R_{m} v$. Again, since $\Lambda_{n}^{m} u_{n}=J_{R_{m}, \lambda_{m, n}}\left(I-\lambda_{m, n} B_{m}\right) \Lambda_{n}^{m-1} u_{n}, n \geq 0, m \in\{1,2, \ldots, N\}$, we have

$$
\Lambda_{n}^{m-1} u_{n}-\lambda_{m, n} B_{m} \Lambda_{n}^{m-1} u_{n} \in\left(I+\lambda_{m, n} R_{m}\right) \Lambda_{n}^{m} u_{n}
$$

that is,

$$
\frac{1}{\lambda_{m, n}}\left(\Lambda_{n}^{m-1} u_{n}-\Lambda_{n}^{m} u_{n}-\lambda_{m, n} B_{m} \Lambda_{n}^{m-1} u_{n}\right) \in R_{m} \Lambda_{n}^{m} u_{n} .
$$

In terms of the monotonicity of $R_{m}$, we get

$$
\left\langle v-\Lambda_{n}^{m} u_{n}, g-B_{m} v-\frac{1}{\lambda_{m, n}}\left(\Lambda_{n}^{m-1} u_{n}-\Lambda_{n}^{m} u_{n}-\lambda_{m, n} B_{m} \Lambda_{n}^{m-1} u_{n}\right)\right\rangle \geq 0,
$$

and hence

$$
\begin{aligned}
\langle v & \left.-\Lambda_{n}^{m} u_{n}, g\right\rangle \\
& \geq\left\langle v-\Lambda_{n}^{m} u_{n}, B_{m} v+\frac{1}{\lambda_{m, n}}\left(\Lambda_{n}^{m-1} u_{n}-\Lambda_{n}^{m} u_{n}-\lambda_{m, n} B_{m} \Lambda_{n}^{m-1} u_{n}\right)\right\rangle \\
& =\left\langle v-\Lambda_{n}^{m} u_{n}, B_{m} v-B_{m} \Lambda_{n}^{m} u_{n}+B_{m} \Lambda_{n}^{m} u_{n}-B_{m} \Lambda_{n}^{m-1} u_{n}+\frac{1}{\lambda_{m, n}}\left(\Lambda_{n}^{m-1} u_{n}-\Lambda_{n}^{m} u_{n}\right)\right\rangle \\
& \geq\left\langle v-\Lambda_{n}^{m} u_{n}, B_{m} \Lambda_{n}^{m} u_{n}-B_{m} \Lambda_{n}^{m-1} u_{n}\right\rangle+\left\langle v-\Lambda_{n}^{m} u_{n}, \frac{1}{\lambda_{m, n}}\left(\Lambda_{n}^{m-1} u_{n}-\Lambda_{n}^{m} u_{n}\right)\right\rangle .
\end{aligned}
$$

In particular,

$$
\begin{aligned}
\left\langle v-\Lambda_{n_{i}}^{m} u_{n_{i}}, g\right\rangle \geq & \left\langle v-\Lambda_{n_{i}}^{m} u_{n_{i}}, B_{m} \Lambda_{n_{i}}^{m} u_{n_{i}}-B_{m} \Lambda_{n_{i}}^{m-1} u_{n_{i}}\right\rangle \\
& +\left\langle v-\Lambda_{n_{i}}^{m} u_{n_{i}}, \frac{1}{\lambda_{m, n_{i}}}\left(\Lambda_{n_{i}}^{m-1} u_{n_{i}}-\Lambda_{n_{i}}^{m} u_{n_{i}}\right)\right\rangle .
\end{aligned}
$$


Since $\left\|\Lambda_{n}^{m} u_{n}-\Lambda_{n}^{m-1} u_{n}\right\| \rightarrow 0$ (due to (3.23)) and $\left\|B_{m} \Lambda_{n}^{m} u_{n}-B_{m} \Lambda_{n}^{m-1} u_{n}\right\| \rightarrow 0$ (due to the Lipschitz continuity of $\left.B_{m}\right)$, we conclude from $\Lambda_{n_{i}}^{m} u_{n_{i}} \rightarrow w$ and $\left\{\lambda_{i, n}\right\} \subset\left[a_{i}, b_{i}\right] \subset\left(0,2 \eta_{i}\right)$ that

$$
\lim _{i \rightarrow \infty}\left\langle v-\Lambda_{n_{i}}^{m} u_{n_{i}}, g\right\rangle=\langle v-w, g\rangle \geq 0 .
$$

It follows from the maximal monotonicity of $B_{m}+R_{m}$ that $0 \in\left(R_{m}+B_{m}\right) w$, that is, $w \in$ $\mathrm{I}\left(B_{m}, R_{m}\right)$. Therefore, $w \in \bigcap_{m=1}^{N} \mathrm{I}\left(B_{m}, R_{m}\right)$.

Next we prove that $w \in \bigcap_{k=1}^{M} \operatorname{GMEP}\left(\Theta_{k}, \varphi_{k}, A_{k}\right)$. Since $\Delta_{n}^{k} x_{n}=T_{r_{k, n}}^{\left(\Theta_{k}, \varphi_{k}\right)}\left(I-r_{k, n} A_{k}\right) \Delta_{n}^{k-1} x_{n}$, $n \geq 0, k \in\{1,2, \ldots, M\}$, we have

$$
\begin{gathered}
\Theta_{k}\left(\Delta_{n}^{k} x_{n}, y\right)+\varphi_{k}(y)-\varphi_{k}\left(\Delta_{n}^{k} x_{n}\right)+\left\langle A_{k} \Delta_{n}^{k-1} x_{n}, y-\Delta_{n}^{k} x_{n}\right\rangle \\
+\frac{1}{r_{k, n}}\left\langle y-\Delta_{n}^{k} x_{n}, \Delta_{n}^{k} x_{n}-\Delta_{n}^{k-1} x_{n}\right\rangle \geq 0 .
\end{gathered}
$$

By (A2), we have

$$
\begin{aligned}
& \varphi_{k}(y)-\varphi_{k}\left(\Delta_{n}^{k} x_{n}\right)+\left\langle A_{k} \Delta_{n}^{k-1} x_{n}, y-\Delta_{n}^{k} x_{n}\right\rangle+\frac{1}{r_{k, n}}\left\langle y-\Delta_{n}^{k} x_{n}, \Delta_{n}^{k} x_{n}-\Delta_{n}^{k-1} x_{n}\right\rangle \\
& \geq \Theta_{k}\left(y, \Delta_{n}^{k} x_{n}\right) .
\end{aligned}
$$

Let $z_{t}=t y+(1-t) w$, for all $t \in(0,1]$ and $y \in C$. This implies that $z_{t} \in C$. Then we have

$$
\begin{aligned}
\left\langle z_{t}-\right. & \left.\Delta_{n}^{k} x_{n}, A_{k} z_{t}\right\rangle \\
\geq & \varphi_{k}\left(\Delta_{n}^{k} x_{n}\right)-\varphi_{k}\left(z_{t}\right)+\left\langle z_{t}-\Delta_{n}^{k} x_{n}, A_{k} z_{t}\right\rangle-\left\langle z_{t}-\Delta_{n}^{k} x_{n}, A_{k} \Delta_{n}^{k-1} x_{n}\right\rangle \\
& \quad-\left\langle z_{t}-\Delta_{n}^{k} x_{n}, \frac{\Delta_{n}^{k} x_{n}-\Delta_{n}^{k-1} x_{n}}{r_{k, n}}\right\rangle+\Theta_{k}\left(z_{t}, \Delta_{n}^{k} x_{n}\right) \\
= & \varphi_{k}\left(\Delta_{n}^{k} x_{n}\right)-\varphi_{k}\left(z_{t}\right)+\left\langle z_{t}-\Delta_{n}^{k} x_{n}, A_{k} z_{t}-A_{k} \Delta_{n}^{k} x_{n}\right\rangle \\
& +\left\langle z_{t}-\Delta_{n}^{k} x_{n}, A_{k} \Delta_{n}^{k} x_{n}-A_{k} \Delta_{n}^{k-1} x_{n}\right\rangle-\left\langle z_{t}-\Delta_{n}^{k} x_{n}, \frac{\Delta_{n}^{k} x_{n}-\Delta_{n}^{k-1} x_{n}}{r_{k, n}}\right\rangle \\
& +\Theta_{k}\left(z_{t}, \Delta_{n}^{k} x_{n}\right) .
\end{aligned}
$$

By (3.26), we have $\left\|A_{k} \Delta_{n}^{k} x_{n}-A_{k} \Delta_{n}^{k-1} x_{n}\right\| \rightarrow 0$ as $n \rightarrow \infty$. Furthermore, by the monotonicity of $A_{k}$, we obtain $\left\langle z_{t}-\Delta_{n}^{k} x_{n}, A_{k} z_{t}-A_{k} \Delta_{n}^{k} x_{n}\right\rangle \geq 0$. Then, by (A4), we obtain

$$
\left\langle z_{t}-w, A_{k} z_{t}\right\rangle \geq \varphi_{k}(w)-\varphi_{k}\left(z_{t}\right)+\Theta_{k}\left(z_{t}, w\right) .
$$

Utilizing (A1), (A4), and (3.39), we obtain

$$
\begin{aligned}
0 & =\Theta_{k}\left(z_{t}, z_{t}\right)+\varphi_{k}\left(z_{t}\right)-\varphi_{k}\left(z_{t}\right) \\
& \leq t \Theta_{k}\left(z_{t}, y\right)+(1-t) \Theta_{k}\left(z_{t}, w\right)+t \varphi_{k}(y)+(1-t) \varphi_{k}(w)-\varphi_{k}\left(z_{t}\right) \\
& \leq t\left[\Theta_{k}\left(z_{t}, y\right)+\varphi_{k}(y)-\varphi_{k}\left(z_{t}\right)\right]+(1-t)\left\langle z_{t}-w, A_{k} z_{t}\right\rangle \\
& =t\left[\Theta_{k}\left(z_{t}, y\right)+\varphi_{k}(y)-\varphi_{k}\left(z_{t}\right)\right]+(1-t) t\left\langle y-w, A_{k} z_{t}\right\rangle,
\end{aligned}
$$


and hence

$$
0 \leq \Theta_{k}\left(z_{t}, y\right)+\varphi_{k}(y)-\varphi_{k}\left(z_{t}\right)+(1-t)\left\langle y-w, A_{k} z_{t}\right\rangle
$$

Letting $t \rightarrow 0$, we have, for each $y \in C$,

$$
0 \leq \Theta_{k}(w, y)+\varphi_{k}(y)-\varphi_{k}(w)+\left\langle y-w, A_{k} w\right\rangle .
$$

This implies that $w \in \operatorname{GMEP}\left(\Theta_{k}, \varphi_{k}, A_{k}\right)$, and hence, $w \in \bigcap_{k=1}^{M} \operatorname{GMEP}\left(\Theta_{k}, \varphi_{k}, A_{k}\right)$. Thus, $w \in \operatorname{Fix}(T) \cap \bigcap_{k=1}^{M} \operatorname{GMEP}\left(\Theta_{k}, \varphi_{k}, A_{k}\right) \cap \bigcap_{m=1}^{N} \mathrm{I}\left(B_{m}, R_{m}\right)$.

Furthermore, let us show that $w \in \Gamma$. In fact, define

$$
\widetilde{T} v= \begin{cases}\nabla f(v)+N_{C} v, & \text { if } v \in C, \\ \emptyset, & \text { if } v \notin C,\end{cases}
$$

where $N_{C} v=\{u \in H:\langle v-x, u\rangle \geq 0, \forall x \in C\}$. Then $\widetilde{T}$ is maximal monotone and $0 \in \widetilde{T} v$ if and only if $v \in \operatorname{VI}(C, \nabla f)$; see [16]. Let $(v, \tilde{v}) \in G(\widetilde{T})$. Then we have $\tilde{v} \in \widetilde{T} v=\nabla f(v)+N_{C} v$, and hence, $\tilde{v}-\nabla f(v) \in N_{C} v$. So, we have $\langle v-x, \tilde{v}-\nabla f(v)\rangle \geq 0$, for all $x \in C$.

On the other hand, from $t_{n}=P_{C}\left(v_{n}-\lambda_{n} \nabla f_{\alpha_{n}}\left(v_{n}\right)\right)$ and $v \in C$, we get $\left\langle v_{n}-\lambda_{n} \nabla f_{\alpha_{n}}\left(v_{n}\right)-\right.$ $\left.t_{n}, t_{n}-v\right\rangle \geq 0$, and hence,

$$
\left\langle v-t_{n}, \frac{t_{n}-v_{n}}{\lambda_{n}}+\nabla f_{\alpha_{n}}\left(v_{n}\right)\right\rangle \geq 0 .
$$

Therefore, from $\tilde{v}-\nabla f(v) \in N_{C} v$ and $t_{n_{i}} \in C$, we have

$$
\begin{aligned}
\left\langle v-t_{n_{i}}, \tilde{v}\right\rangle \geq & \left\langle v-t_{n_{i}}, \nabla f(v)\right\rangle \\
\geq & \left\langle v-t_{n_{i}}, \nabla f(v)\right\rangle-\left\langle v-t_{n_{i}}, \frac{t_{n_{i}}-v_{n_{i}}}{\lambda_{n_{i}}}+\nabla f_{\alpha_{n_{i}}}\left(v_{n_{i}}\right)\right\rangle \\
= & \left\langle v-t_{n_{i}}, \nabla f(v)\right\rangle-\left\langle v-t_{n_{i}}, \frac{t_{n_{i}}-v_{n_{i}}}{\lambda_{n_{i}}}+\nabla f\left(v_{n_{i}}\right)\right\rangle-\alpha_{n_{i}}\left\langle v-t_{n_{i}}, v_{n_{i}}\right\rangle \\
= & \left\langle v-t_{n_{i}}, \nabla f(v)-\nabla f\left(t_{n_{i}}\right)\right\rangle+\left\langle v-t_{n_{i}}, \nabla f\left(t_{n_{i}}\right)-\nabla f\left(v_{n_{i}}\right)\right\rangle \\
& -\left\langle v-t_{n_{i}}, \frac{t_{n_{i}}-v_{n_{i}}}{\lambda_{n_{i}}}\right\rangle-\alpha_{n_{i}}\left\langle v-t_{n_{i}}, v_{n_{i}}\right\rangle \\
\geq & \left\langle v-t_{n_{i}}, \nabla f\left(t_{n_{i}}\right)-\nabla f\left(v_{n_{i}}\right)\right\rangle-\left\langle v-t_{n_{i}}, \frac{t_{n_{i}}-v_{n_{i}}}{\lambda_{n_{i}}}\right\rangle-\alpha_{n_{i}}\left\langle v-t_{n_{i}}, v_{n_{i}}\right\rangle .
\end{aligned}
$$

Hence, it is easy to see that $\langle v-w, \tilde{v}\rangle \geq 0$ as $i \rightarrow \infty$. Since $\widetilde{T}$ is maximal monotone, we have $w \in \widetilde{T}^{-1} 0$, and hence, $w \in \operatorname{VI}(C, \nabla f)=\Gamma$. Consequently, $w \in \bigcap_{k=1}^{M} \operatorname{GMEP}\left(\Theta_{k}, \varphi_{k}, A_{k}\right) \cap$ $\bigcap_{i=1}^{N} \mathrm{I}\left(B_{i}, R_{i}\right) \cap \operatorname{Fix}(T) \cap \Gamma=: \Omega$. This shows that $\omega_{w}\left(x_{n}\right) \subset \Omega$.

Step 5. We prove that $\omega_{w}\left(x_{n}\right) \subset \Xi$.

Indeed, utilizing Lemmas 2.6 and 2.4, from (3.1) and (3.6), we find that, for all $p \in \Omega$,

$$
\begin{aligned}
& \left\|x_{n+1}-p\right\|^{2} \\
& \quad=\left\|\epsilon_{n} \gamma\left(\delta_{n} V x_{n}+\left(1-\delta_{n}\right) S x_{n}\right)+\left(I-\epsilon_{n} \mu F\right) y_{n}-p\right\|^{2}
\end{aligned}
$$




$$
\begin{aligned}
& =\left\|\epsilon_{n} \gamma\left(\delta_{n} V x_{n}+\left(1-\delta_{n}\right) S x_{n}\right)-\epsilon_{n} \mu F p+\left(I-\epsilon_{n} \mu F\right) y_{n}-\left(I-\epsilon_{n} \mu F\right) p\right\|^{2} \\
& =\left\|\epsilon_{n}\left[\delta_{n}\left(\gamma V x_{n}-\mu F p\right)+\left(1-\delta_{n}\right)\left(\gamma S x_{n}-\mu F p\right)\right]+\left(I-\epsilon_{n} \mu F\right) y_{n}-\left(I-\epsilon_{n} \mu F\right) p\right\|^{2} \\
& =\| \epsilon_{n}\left[\delta_{n}\left(\gamma V x_{n}-\gamma V p\right)+\left(1-\delta_{n}\right)\left(\gamma S x_{n}-\gamma S p\right)\right]+\left(I-\epsilon_{n} \mu F\right) y_{n}-\left(I-\epsilon_{n} \mu F\right) p \\
& +\epsilon_{n}\left[\delta_{n}(\gamma V p-\mu F p)+\left(1-\delta_{n}\right)(\gamma S p-\mu F p)\right] \|^{2} \\
& \leq\left\|\epsilon_{n}\left[\delta_{n}\left(\gamma V x_{n}-\gamma V p\right)+\left(1-\delta_{n}\right)\left(\gamma S x_{n}-\gamma S p\right)\right]+\left(I-\epsilon_{n} \mu F\right) y_{n}-\left(I-\epsilon_{n} \mu F\right) p\right\|^{2} \\
& +2 \epsilon_{n} \delta_{n}\left\langle(\gamma V p-\mu F p), x_{n+1}-p\right\rangle+2 \epsilon_{n}\left(1-\delta_{n}\right)\left\langle(\gamma S p-\mu F p), x_{n+1}-p\right\rangle \\
& \leq\left[\epsilon_{n}\left\|\delta_{n}\left(\gamma V x_{n}-\gamma V p\right)+\left(1-\delta_{n}\right)\left(\gamma S x_{n}-\gamma S p\right)\right\|+\left\|\left(I-\epsilon_{n} \mu F\right) y_{n}-\left(I-\epsilon_{n} \mu F\right) p\right\|\right]^{2} \\
& +2 \epsilon_{n} \delta_{n}\left\langle(\gamma V p-\mu F p), x_{n+1}-p\right\rangle+2 \epsilon_{n}\left(1-\delta_{n}\right)\left\langle(\gamma S p-\mu F p), x_{n+1}-p\right\rangle \\
& \leq\left[\epsilon_{n}\left(\delta_{n} \gamma \rho\left\|x_{n}-p\right\|+\left(1-\delta_{n}\right) \gamma\left\|x_{n}-p\right\|\right)+\left(1-\epsilon_{n} \tau\right)\left\|y_{n}-p\right\|\right]^{2} \\
& +2 \epsilon_{n} \delta_{n}\left\langle(\gamma V p-\mu F p), x_{n+1}-p\right\rangle+2\left(1-\delta_{n}\right) \epsilon_{n}\left\langle(\gamma S p-\mu F p), x_{n+1}-p\right\rangle \\
& =\left[\epsilon_{n}\left(1-\delta_{n}(1-\rho)\right) \gamma\left\|x_{n}-p\right\|+\left(1-\epsilon_{n} \tau\right)\left\|y_{n}-p\right\|\right]^{2} \\
& +2 \epsilon_{n} \delta_{n}\left\langle(\gamma V p-\mu F p), x_{n+1}-p\right\rangle+2 \epsilon_{n}\left(1-\delta_{n}\right)\left\langle(\gamma S p-\mu F p), x_{n+1}-p\right\rangle \\
& \leq\left[\epsilon_{n}\left(1-\delta_{n}(1-\rho)\right) \gamma\left\|x_{n}-p\right\|+\left(1-\epsilon_{n} \tau\right)\left(\left\|x_{n}-p\right\|+\alpha_{n} b\|p\|\right)\right]^{2} \\
& +2 \epsilon_{n} \delta_{n}\left\langle(\gamma V p-\mu F p), x_{n+1}-p\right\rangle+2 \epsilon_{n}\left(1-\delta_{n}\right)\left\langle(\gamma S p-\mu F p), x_{n+1}-p\right\rangle \\
& \leq\left[\epsilon_{n}\left(1-\delta_{n}(1-\rho)\right) \gamma\left(\left\|x_{n}-p\right\|+\alpha_{n} b\|p\|\right)+\left(1-\epsilon_{n} \tau\right)\left(\left\|x_{n}-p\right\|+\alpha_{n} b\|p\|\right)\right]^{2} \\
& +2 \epsilon_{n} \delta_{n}\left\langle(\gamma V p-\mu F p), x_{n+1}-p\right\rangle+2 \epsilon_{n}\left(1-\delta_{n}\right)\left\langle(\gamma S p-\mu F p), x_{n+1}-p\right\rangle \\
& =\left(1-\epsilon_{n}(\tau-\gamma)-\epsilon_{n} \delta_{n}(1-\rho) \gamma\right)^{2}\left(\left\|x_{n}-p\right\|+\alpha_{n} b\|p\|\right)^{2} \\
& +2 \epsilon_{n} \delta_{n}\left\langle(\gamma V p-\mu F p), x_{n+1}-p\right\rangle+2 \epsilon_{n}\left(1-\delta_{n}\right)\left\langle(\gamma S p-\mu F p), x_{n+1}-p\right\rangle \\
& \leq\left(1-\epsilon_{n}(\tau-\gamma)-\epsilon_{n} \delta_{n}(1-\rho) \gamma\right)\left(\left\|x_{n}-p\right\|+\alpha_{n} b\|p\|\right)^{2} \\
& +2 \epsilon_{n} \delta_{n}\left\langle(\gamma V p-\mu F p), x_{n+1}-p\right\rangle+2 \epsilon_{n}\left(1-\delta_{n}\right)\left\langle(\gamma S p-\mu F p), x_{n+1}-p\right\rangle \\
& \leq\left(1-\epsilon_{n} \delta_{n}(1-\rho) \gamma\right)\left[\left\|x_{n}-p\right\|^{2}+\alpha_{n} b\|p\|\left(2\left\|x_{n}-p\right\|+\alpha_{n} b\|p\|\right)\right] \\
& +2 \epsilon_{n} \delta_{n}\left\langle(\gamma V p-\mu F p), x_{n+1}-p\right\rangle+2 \epsilon_{n}\left(1-\delta_{n}\right)\left\langle(\gamma S p-\mu F p), x_{n+1}-p\right\rangle \\
& \leq\left(1-\epsilon_{n} \delta_{n}(1-\rho) \gamma\right)\left\|x_{n}-p\right\|^{2}+2 \epsilon_{n} \delta_{n}\left\langle(\gamma V p-\mu F p), x_{n+1}-p\right\rangle \\
& +2 \epsilon_{n}\left(1-\delta_{n}\right)\left\langle(\gamma S p-\mu F p), x_{n+1}-p\right\rangle+\alpha_{n} b\|p\|\left(2\left\|x_{n}-p\right\|+\alpha_{n} b\|p\|\right) .
\end{aligned}
$$

Take an arbitrary $w \in \omega_{w}\left(x_{n}\right)$. Then there exists a subsequence $\left\{x_{n_{i}}\right\}$ of $\left\{x_{n}\right\}$ such that $x_{n_{i}} \rightarrow w$. Utilizing (3.40), we obtain, for all $p \in \Omega$,

$$
\begin{aligned}
\| x_{n+1} & -p \|^{2} \\
\leq & \left(1-\epsilon_{n}(\tau-\gamma)-\epsilon_{n} \delta_{n}(1-\rho) \gamma\right)\left(\left\|x_{n}-p\right\|+\alpha_{n} b\|p\|\right)^{2} \\
\quad & +2 \epsilon_{n} \delta_{n}\left\langle(\gamma V p-\mu F p), x_{n+1}-p\right\rangle+2 \epsilon_{n}\left(1-\delta_{n}\right)\left\langle(\gamma S p-\mu F p), x_{n+1}-p\right\rangle \\
\leq & \left(\left\|x_{n}-p\right\|+\alpha_{n} b\|p\|\right)^{2}+2 \epsilon_{n} \delta_{n}\left\langle(\gamma V p-\mu F p), x_{n+1}-p\right\rangle \\
\quad & +2 \epsilon_{n}\left(1-\delta_{n}\right)\left\langle(\gamma S p-\mu F p), x_{n+1}-p\right\rangle,
\end{aligned}
$$


which implies that

$$
\begin{aligned}
&\left\langle(\mu F-\gamma S) p, x_{n}-p\right\rangle \\
& \leq\left\langle(\mu F-\gamma S) p, x_{n}-x_{n+1}\right\rangle+\left\langle(\mu F-\gamma S) p, x_{n+1}-p\right\rangle \\
& \leq\|(\mu F-\gamma S) p\|\left\|x_{n}-x_{n+1}\right\|+\frac{\left(\left\|x_{n}-p\right\|+\alpha_{n} b\|p\|\right)^{2}-\left\|x_{n+1}-p\right\|^{2}}{2 \epsilon_{n}\left(1-\delta_{n}\right)} \\
& \quad+\frac{\delta_{n}}{1-\delta_{n}}\left\langle(\gamma V-\mu F) p, x_{n+1}-p\right\rangle \\
& \leq\|(\mu F-\gamma S) p\|\left\|x_{n}-x_{n+1}\right\| \\
&+\frac{\left(\left\|x_{n}-x_{n+1}\right\|+\alpha_{n} b\|p\|\right)\left(\left\|x_{n}-p\right\|+\left\|x_{n+1}-p\right\|+\alpha_{n} b\|p\|\right)}{2 \epsilon_{n}\left(1-\delta_{n}\right)} \\
&+\frac{\delta_{n}}{1-\delta_{n}}\|(\gamma V-\mu F) p\|\left\|x_{n+1}-p\right\| .
\end{aligned}
$$

Since the combination of the boundedness of $\left\{x_{n}\right\}, \delta_{n} \rightarrow 0, \alpha_{n}=o\left(\epsilon_{n}^{2}\right)$, and $\left\|x_{n}-x_{n+1}\right\|=$ $o\left(\epsilon_{n}\right)$ (due to Step 2) implies that

$$
\lim _{n \rightarrow \infty} \frac{\left(\left\|x_{n}-x_{n+1}\right\|+\alpha_{n} b\|p\|\right)\left(\left\|x_{n}-p\right\|+\left\|x_{n+1}-p\right\|+\alpha_{n} b\|p\|\right)}{2 \epsilon_{n}\left(1-\delta_{n}\right)}=0,
$$

from (3.41), we conclude that

$$
\begin{aligned}
\langle(\mu F-\gamma S) p, w-p\rangle & =\lim _{i \rightarrow \infty}\left\langle(\mu F-\gamma S) p, x_{n_{i}}-p\right\rangle \\
& \leq \limsup _{n \rightarrow \infty}\left\langle(\mu F-\gamma S) p, x_{n}-p\right\rangle \\
& \leq 0, \quad \forall p \in \Omega,
\end{aligned}
$$

that is,

$$
\langle(\mu F-\gamma S) p, w-p\rangle \leq 0, \quad \forall p \in \Omega .
$$

Since $\mu F-\gamma S$ is $(\mu \eta-\gamma)$-strongly monotone and $(\mu \kappa+\gamma)$-Lipschitz continuous, by Minty's lemma [41] we know that (3.42) is equivalent to the VIP

$$
\langle(\mu F-\gamma S) w, p-w\rangle \geq 0, \quad \forall p \in \Omega
$$

So, it follows that $w \in \operatorname{VI}(\Omega, \mu F-\gamma S)=: \Xi$. This shows that $\omega_{w}\left(x_{n}\right) \subset \Xi$.

Step 6. We prove that $x_{n} \rightarrow x^{*}$ where $\left\{x^{*}\right\}=\operatorname{VI}(\Xi, \mu F-\gamma V)$.

Indeed, note that $\left\{x^{*}\right\}=\operatorname{VI}(\Xi, \mu F-\gamma V)$. Since $\left\{x_{n}\right\}$ is bounded and $H$ is reflexive, there exists a subsequence $\left\{x_{n_{i}}\right\}$ of $\left\{x_{n}\right\}$ such that $x_{n_{i}} \rightarrow w$ and

$$
\limsup _{n \rightarrow \infty}\left\langle(\gamma V-\mu F) x^{*}, x_{n}-x^{*}\right\rangle=\limsup _{i \rightarrow \infty}\left\langle(\gamma V-\mu F) x^{*}, x_{n_{i}}-x^{*}\right\rangle=\left\langle(\gamma V-\mu F) x^{*}, w-x^{*}\right\rangle .
$$

According to Step 5, we get $w \in \Xi$. So, it follows from $\left\{x^{*}\right\}=\operatorname{VI}(\Xi, \mu F-\gamma V)$ that

$$
\limsup _{n \rightarrow \infty}\left\langle(\gamma V-\mu F) x^{*}, x_{n}-x^{*}\right\rangle=\left\langle(\gamma V-\mu F) x^{*}, w-x^{*}\right\rangle \leq 0 .
$$


However, from $x^{*} \in \Xi \subset \Omega$ and condition (C10), we deduce that, for sufficiently large $n \geq 0$,

$$
\begin{aligned}
& \left\langle(\gamma S-\mu F) x^{*}, x_{n+1}-x^{*}\right\rangle \\
& \quad=\left\langle(\gamma S-\mu F) x^{*}, x_{n+1}-P_{\Omega} x_{n+1}\right\rangle+\left\langle(\gamma S-\mu F) x^{*}, P_{\Omega} x_{n+1}-x^{*}\right\rangle \\
& \quad \leq\left\langle(\gamma S-\mu F) x^{*}, x_{n+1}-P_{\Omega} x_{n+1}\right\rangle \\
& \quad \leq\left\|(\gamma S-\mu F) x^{*}\right\| d\left(x_{n+1}, \Omega\right) \\
& \quad \leq\left\|(\gamma S-\mu F) x^{*}\right\|\left(\frac{1}{\bar{k}}\left\|x_{n+1}-T x_{n+1}\right\|\right)^{1 / \theta} .
\end{aligned}
$$

Utilizing Lemma 2.1(a), we have, for sufficiently large $n \geq 0$,

$$
\begin{aligned}
&\left\|x_{n+1}-T x_{n+1}\right\| \\
& \leq\left\|x_{n+1}-T x_{n}\right\|+\left\|T x_{n}-T x_{n+1}\right\| \\
& \leq \frac{1+\xi}{1-\xi}\left\|x_{n}-x_{n+1}\right\|+\left\|\epsilon_{n} \gamma\left(\delta_{n} V x_{n}+\left(1-\delta_{n}\right) S x_{n}\right)+\left(I-\epsilon_{n} \mu F\right) y_{n}-T x_{n}\right\| \\
& \leq \frac{1+\xi}{1-\xi}\left\|x_{n}-x_{n+1}\right\|+\left\|y_{n}-T x_{n}\right\|+\epsilon_{n}\left\|\gamma\left(\delta_{n} V x_{n}+\left(1-\delta_{n}\right) S x_{n}\right)-\mu F y_{n}\right\| \\
& \leq \frac{1+\xi}{1-\xi}\left\|x_{n}-x_{n+1}\right\|+\left\|y_{n}-x_{n}\right\|+\left\|x_{n}-T x_{n}\right\|+\epsilon_{n}\left\|\gamma\left(\delta_{n} V x_{n}+\left(1-\delta_{n}\right) S x_{n}\right)-\mu F y_{n}\right\| \\
& \leq \frac{1+\xi}{1-\xi}\left\|x_{n}-x_{n+1}\right\|+\left\|y_{n}-x_{n}\right\|+\left\|x_{n}-T t_{n}\right\|+\left\|T t_{n}-T x_{n}\right\| \\
&+\epsilon_{n}\left\|\gamma \delta_{n}\left(V x_{n}-S x_{n}\right)+\gamma S x_{n}-\mu F y_{n}\right\| \\
& \leq \frac{1+\xi}{1-\xi}\left\|x_{n}-x_{n+1}\right\|+\left\|y_{n}-x_{n}\right\|+\left\|x_{n}-t_{n}\right\|+\left\|t_{n}-T t_{n}\right\| \\
&+\left\|T t_{n}-T x_{n}\right\|+\epsilon_{n}\left\|\gamma \delta_{n}\left(V x_{n}-S x_{n}\right)+\gamma S x_{n}-\mu F y_{n}\right\| \\
& \leq \frac{1+\xi}{1-\xi}\left\|x_{n}-x_{n+1}\right\|+\left\|y_{n}-x_{n}\right\|+\left(1+\frac{1+\xi}{1-\xi}\right)\left\|t_{n}-x_{n}\right\|+\left\|t_{n}-T t_{n}\right\|+\epsilon_{n} \widetilde{M}_{4}, \quad(3.45)
\end{aligned}
$$

where $\widetilde{M}_{4}=\sup _{n \geq 0}\left\|\gamma \delta_{n}\left(V x_{n}-S x_{n}\right)+\gamma S x_{n}-\mu F y_{n}\right\|<\infty$. Hence, for a large enough constant $\bar{k}_{1}>0$, from (3.44) and (3.45), we have, for sufficiently large $n \geq 0$,

$$
\begin{aligned}
\left\langle(\gamma S-\mu F) x^{*}, x_{n+1}-x^{*}\right\rangle & \\
\leq & \left\|(\gamma S-\mu F) x^{*}\right\|\left(\frac{1}{\bar{k}}\left\|x_{n+1}-T x_{n+1}\right\|\right)^{1 / \theta} \\
\leq & \left\|(\gamma S-\mu F) x^{*}\right\|\left\{\frac { 1 } { \overline { k } } \left[\frac{1+\xi}{1-\xi}\left\|x_{n}-x_{n+1}\right\|+\left\|y_{n}-x_{n}\right\|+\left(1+\frac{1+\xi}{1-\xi}\right)\left\|t_{n}-x_{n}\right\|\right.\right. \\
& \left.\left.+\left\|t_{n}-T t_{n}\right\|+\epsilon_{n} \tilde{M}_{4}\right]\right\}^{1 / \theta} \\
\leq & \bar{k}_{1}\left(\epsilon_{n}+\left\|x_{n}-x_{n+1}\right\|+\left\|y_{n}-x_{n}\right\|+\left\|x_{n}-t_{n}\right\|+\left\|t_{n}-T t_{n}\right\|\right)^{1 / \theta} \\
\leq & \bar{k}_{1} \epsilon_{n}^{1 / \theta}\left(1+\frac{\left\|x_{n}-x_{n+1}\right\|+\left\|y_{n}-x_{n}\right\|+\left\|x_{n}-t_{n}\right\|+\left\|t_{n}-T t_{n}\right\|}{\epsilon_{n}}\right)^{1 / \theta} .
\end{aligned}
$$


Next we prove that $\lim _{n \rightarrow \infty}\left\|x_{n}-x^{*}\right\|=0$. As a matter of fact, putting $p=x^{*}$ in (3.40), we obtain from (3.46) that

$$
\begin{aligned}
\left\|x_{n+1}-x^{*}\right\|^{2} & \\
\leq & \left(1-\epsilon_{n} \delta_{n}(1-\rho) \gamma\right)\left\|x_{n}-x^{*}\right\|^{2}+2 \epsilon_{n} \delta_{n}\left\langle(\gamma V-\mu F) x^{*}, x_{n+1}-x^{*}\right\rangle \\
& +2 \epsilon_{n}\left(1-\delta_{n}\right)\left\langle(\gamma S-\mu F) x^{*}, x_{n+1}-x^{*}\right\rangle+\alpha_{n} b\left\|x^{*}\right\|\left(2\left\|x_{n}-x^{*}\right\|+\alpha_{n} b\left\|x^{*}\right\|\right) \\
\leq & \left(1-\epsilon_{n} \delta_{n}(1-\rho) \gamma\right)\left\|x_{n}-x^{*}\right\|^{2}+\epsilon_{n} \delta_{n}(1-\rho) \gamma \cdot \frac{2}{(1-\rho) \gamma}\left[\left\langle(\gamma V-\mu F) x^{*}, x_{n+1}-x^{*}\right\rangle\right. \\
& \left.+\left(1-\delta_{n}\right) \frac{\left\langle(\gamma S-\mu F) x^{*}, x_{n+1}-x^{*}\right\rangle}{\delta_{n}}\right]+\alpha_{n} b\left\|x^{*}\right\|\left(2\left\|x_{n}-x^{*}\right\|+\alpha_{n} b\left\|x^{*}\right\|\right) \\
\leq & \left(1-\epsilon_{n} \delta_{n}(1-\rho) \gamma\right)\left\|x_{n}-x^{*}\right\|^{2}+\epsilon_{n} \delta_{n}(1-\rho) \gamma \cdot \frac{2}{(1-\rho) \gamma}\left[\left\langle(\gamma V-\mu F) x^{*}, x_{n+1}-x^{*}\right\rangle\right. \\
& \left.+\bar{k}_{1} \frac{\epsilon_{n}^{1 / \theta}}{\delta_{n}}\left(1+\frac{\left\|x_{n}-x_{n+1}\right\|+\left\|y_{n}-x_{n}\right\|+\left\|x_{n}-t_{n}\right\|+\left\|t_{n}-T t_{n}\right\|}{\epsilon_{n}}\right)^{1 / \theta}\right] \\
& +\alpha_{n} b\left\|x^{*}\right\|\left(2\left\|x_{n}-x^{*}\right\|+\alpha_{n} b\left\|x^{*}\right\|\right) .
\end{aligned}
$$

Since $\sum_{n=1}^{\infty} \epsilon_{n} \delta_{n}=\infty, \sum_{n=1}^{\infty} \alpha_{n}<\infty, \frac{\epsilon_{n}^{1 / \theta}}{\delta_{n}} \rightarrow 0,\left\|x_{n}-y_{n}\right\|=o\left(\epsilon_{n}^{2}\right)$, and $\left\|x_{n}-x_{n+1}\right\|=o\left(\epsilon_{n}\right)$, we conclude from (3.36), (3.37), and $x_{n} \rightarrow x^{*}$ that $\sum_{n=1}^{\infty} \epsilon_{n} \delta_{n}(1-\rho) \gamma=\infty, \sum_{n=1}^{\infty} \alpha_{n} b\left\|x^{*}\right\| \times$ $\left(2\left\|x_{n}-x^{*}\right\|+\alpha_{n} b\left\|x^{*}\right\|\right) \leq \infty$, and

$$
\begin{aligned}
& \limsup _{n \rightarrow \infty} \frac{2}{(1-\rho) \gamma}\left[\left\langle(\gamma V-\mu F) x^{*}, x_{n+1}-x^{*}\right\rangle\right. \\
& \left.\quad+\bar{k}_{1} \frac{\epsilon_{n}^{1 / \theta}}{\delta_{n}}\left(1+\frac{\left\|x_{n}-x_{n+1}\right\|+\left\|y_{n}-x_{n}\right\|+\left\|x_{n}-t_{n}\right\|+\left\|t_{n}-T t_{n}\right\|}{\epsilon_{n}}\right)^{1 / \theta}\right] \leq 0 .
\end{aligned}
$$

Therefore, applying Lemma 2.8 to (3.47), we infer that $\lim _{n \rightarrow \infty}\left\|x_{n}-x^{*}\right\|=0$. This completes the proof.

Remark 3.1 Algorithm 3.1 and Theorem 3.1 extend and generalize algorithms and convergence results in $[28,30]$.

\section{Competing interests}

The authors declare that there is no conflict of interests regarding the publication of this article.

\section{Authors' contributions}

All authors contributed equally and significantly in writing this article. All authors read and approved the final manuscript.

\section{Author details}

${ }^{1}$ Department of Mathematics, Shanghai Normal University, Shanghai, 200234, China. ${ }^{2}$ Department of Information Management, Innovation Center for Big Data and Digital Convergence, Yuan Ze University, Chung-Li, 32003, Taiwan. ${ }^{3}$ Center for Fundamental Science, Kaohsiung Medical University, Kaohsiung, 80708, Taiwan.

\section{Acknowledgements}

In this research, the first author was partially supported by the National Science Foundation of China (11071169), Innovation Program of Shanghai Municipal Education Commission (09ZZ133), and PhD Program Foundation of Ministry of Education of China (20123127110002). The second author and third author were supported partly by the National Science Council of the Republic of China. 
References

1. Ansari, QH: Metric Spaces - Including Fixed Point Theory and Set-Valued Maps. Narosa Publishing House, New Delhi (2010)

2. Bianchi, M, Schaible, S: Generalized monotone bifunctions and equilibrium problems. J. Optim. Theory Appl. 90 31-43 (1996)

3. Bianchi, M, Schaible, S: Equilibrium problems under generalized convexity and generalized monotonicity. J. Glob. Optim. 30, 121-134 (2004)

4. Blum, E, Oettli, W: From optimization and variational inequalities to equilibrium problems. Math. Stud. 63(1-4), 123-145 (1994)

5. Flores-Bazán, F: Existence theorems for generalized noncoercive equilibrium problems: the quasi-convex case. SIAM J. Optim. 11, 675-690 (2000)

6. Flores-Bazán, F: Existence theory for finite-dimensional pseudomonotone equilibrium problems. Acta Appl. Math. 77 249-297 (2003)

7. Ansari, QH, Lalitha, CS, Mehta, M: Generalized Convexity, Nonsmooth Variational Inequalities and Nonsmooth Optimization. CRC Press, Boca Raton (2014)

8. Facchinei, F, Pang, J-S: Finite-Dimensional Variational Inequalities and Complementarity Problems, vol. I. Springer, New York (2003)

9. Facchinei, F, Pang, J-S: Finite-Dimensional Variational Inequalities and Complementarity Problems, vol. II. Springer New York (2003)

10. Glowinski, R: Numerical Methods for Nonlinear Variational Problems. Springer, New York (1984)

11. Kinderlehrer, D, Stampacchia, G: An Introduction to Variational Inequalities and Their Applications. Academic Press, New York (1980)

12. Nadezhkina, N, Takahashi, W: Weak convergence theorem by an extragradient method for nonexpansive mappings and monotone mappings. J. Optim. Theory Appl. 128, 191-201 (2006)

13. Takahashi, S, Takahashi, W: Strong convergence theorem for a generalized equilibrium problem and a nonexpansive mapping in a Hilbert space. Nonlinear Anal. 69, 1025-1033 (2008)

14. Ansari, QH, Wong, N-C, Yao, J-C: The existence of nonlinear inequalities. Appl. Math. Lett. 12(5), 89-92 (1999)

15. Gwinner, J: Stability of monotone variational inequalities with various applications. In: Giannessi, F, Maugeri, A (eds.) Variational Inequalities and Network Equilibrium Problems, pp. 123-142. Plenum, New York (1995)

16. Rockafellar, RT: Monotone operators and the proximal point algorithms. SIAM J. Control Optim. 14, $877-898$ (1976)

17. Huang, N-J: A new completely general class of variational inclusions with noncompact valued mappings. Comput. Math. Appl. 35(10), 9-14 (1998)

18. Zeng, L-C, Guu, S-M, Yao, J-C: Characterization of H-monotone operators with applications to variational inclusions Comput. Math. Appl. 50(3-4), 329-337 (2005)

19. Ceng, L-C, Al-Homidan, S: Algorithms of common solutions for generalized mixed equilibria, variational inclusions, and constrained convex minimization. Abstr. Appl. Anal. 2014, Article ID 132053 (2014)

20. Ceng, L-C, Ansari, QH, Wong, MM, Yao, J-C: Mann type hybrid extragradient method for variational inequalities, variational inclusions and fixed point problems. Fixed Point Theory 13(2), 403-422 (2012)

21. Ceng, L-C, Ansari, QH, Schaible, S: Hybrid extragradient-like methods for generalized mixed equilibrium problems, system of generalized equilibrium problems and optimization problems. J. Glob. Optim. 53, 69-96 (2012)

22. Ceng, L-C, Ansari, QH, Yao, J-C: Relaxed extragradient iterative methods for variational inequalities. Appl. Math. Comput. 218, 1112-1123 (2011)

23. Byrne, C: Iterative oblique projection onto convex sets and the split feasibility problem. Inverse Problems 18(2), 441-453 (2002)

24. Censor, Y, Bortfeld, T, Martin, B, Trofimov, A: A unified approach for inversion problems in intensity-modulated radiation therapy. Phys. Med. Biol. 51, 2353-2365 (2006)

25. Censor, Y, Motova, A, Segal, A: Perturbed projections and subgradient projections for the multi-sets split feasibility problem. J. Math. Anal. Appl. 327(2), 1244-1256 (2007)

26. Ansari, QH, Rehan, A: Split feasibility and fixed point problems. In: Ansari, QH (ed.) Nonlinear Analysis: Approximation Theory, Optimization and Applications, pp. 281-322. Birkhäuser, Basel (2014)

27. Ceng, L-C, Ansari, QH, Yao, J-C: An extragradient method for solving split feasibility and fixed point problems. Comput. Math. Appl. 64, 633-642 (2012)

28. Ceng, L-C, Ansari, OH, Yao, J-C: Relaxed extragradient methods for finding minimum-norm solutions of the split feasibility problem. Nonlinear Anal. 75, 2116-2125 (2012)

29. Ansari, QH, Ceng, L-C, Gupta, H: Triple hierarchical variational inequalities. In: Ansari, QH (ed.) Nonlinear Analysis: Approximation Theory, Optimization and Applications, pp. 231-280. Birkhäuser, Basel (2014)

30. Kong, Z-R, Ceng, L-C, Ansari, QH, Pang, C-T: Multistep hybrid extragradient method for triple hierarchical variational inequalities. Abstr. Appl. Anal. 2013, Article ID 718624 (2013)

31. Byrne, C: A unified treatment of some iterative algorithms in signal processing and image reconstruction. Inverse Problems 20, 103-120 (2004)

32. Combettes, PL: Solving monotone inclusions via compositions of nonexpansive averaged operators. Optimization 53(5-6), 475-504 (2004)

33. Marino, G, Xu, H-K: Weak and strong convergence theorems for strict pseudo-contractions in Hilbert spaces. J. Math. Anal. Appl. 329, 336-346 (2007)

34. Yao, Y, Liou, Y-C, Kang, SM: Approach to common elements of variational inequality problems and fixed point problems via a relaxed extragradient method. Comput. Math. Appl. 59, 3472-3480 (2010)

35. Han, D, Lo, HK: Solving non-additive traffic assignment problems: a descent method for co-coercive variational inequalities. Eur. J. Oper. Res. 159, 529-544 (2004)

36. $\mathrm{Xu}, \mathrm{H}-\mathrm{K}, \mathrm{Kim}, \mathrm{T}-\mathrm{H}$ : Convergence of hybrid steepest-descent methods for variational inequalities. J. Optim. Theory Appl. 119(1), 185-201 (2003)

37. Ceng, L-C, Yao, J-C: A hybrid iterative scheme for mixed equilibrium problems and fixed point problems. J. Comput. Appl. Math. 214, 186-201 (2008) 
38. Xu, H-K: Iterative algorithms for nonlinear operators. J. Lond. Math. Soc. 66(1), 240-256 (2002)

39. Barbu, V: Nonlinear Semigroups and Differential Equations in Banach Spaces. Noordhoff, Groningen (1976)

40. $\mathrm{Xu}, \mathrm{H}-\mathrm{K}$ : Iterative methods for the split feasibility problem in infinite-dimensional Hilbert spaces. Inverse Problems 26 Article ID 105018 (2010)

41. Goebel, K, Kirk, WA: Topics on Metric Fixed Point Theory. Cambridge University Press, Cambridge (1990)

10.1186/1029-242X-2014-492

Cite this article as: Ceng et al.: Multi-step extragradient method with regularization for triple hierarchical variational inequalities with variational inclusion and split feasibility constraints. Journal of Inequalities and Applications 2014, 2014:492

Submit your manuscript to a SpringerOpen ${ }^{\circ}$ journal and benefit from:

- Convenient online submission

- Rigorous peer review

- Immediate publication on acceptance

- Open access: articles freely available online

- High visibility within the field

- Retaining the copyright to your article 